\title{
ON THE FREYD CATEGORIES OF AN ADDITIVE CATEGORY
}

\author{
APOSTOLOS BELIGIANNIS
}

(communicated by Charles Weibel)

\begin{abstract}
To any additive category $\mathfrak{C}$, we associate in a functorial way two additive categories $\mathcal{A}(\mathfrak{C}), \mathcal{B}(\mathfrak{C})$. The category $\mathcal{A}(\mathfrak{C})$, resp. $\mathcal{B}(\mathfrak{C})$, is the reflection of $\mathfrak{C}$ in the category of additive categories with cokernels, resp. kernels, and cokernel, resp. kernel, preserving functors. Then the iteration $\mathcal{A B}(\mathfrak{C})$ is the reflection of $\mathfrak{C}$ in the category of abelian categories and exact functors. We call $\mathcal{A}(\mathfrak{C})$ and $\mathcal{B}(\mathfrak{C})$ the Freyd categories of $\mathfrak{C}$ since the first systematic study of these categories was done by Freyd in the mid-sixties. The purpose of the paper is to study further the Freyd categories and to indicate their applications to the module theory of an abelian or triangulated category.
\end{abstract}

\section{Introduction}

The notion of a contravariantly, resp. covariantly, finite subcategory of an additive category, was introduced by Freyd $[\mathbf{2 0}]$ under the name ample, resp. coample, subcategory and rediscovered later by Auslander and Smalø [9], see also [18]. Auslander and Smalø coined the terminology contravariantly, resp. covariantly, finite, motivated by the fact that these categories admit certain finiteness conditions on their categories of functors. These notions play an important role in relative homology and modern representation theory. Our main purpose in this paper is to study certain factor categories of the category of morphisms of an additive category, induced by nice contravariantly or covariantly finite subcategories, and to indicate their applications to the module theory of an abelian or triangulated category.

Suppose throughout that $\mathfrak{C}$ is an additive category and consider in the morphism category $\mathfrak{C}^{2}$ of $\mathfrak{C}$, the full subcategory $\mathfrak{X}$ consisting of split monics and the full subcategory $\mathfrak{Y}$ of split epics. The subcategory $\mathfrak{X}$, resp. $\mathfrak{Y}$, is a primal example of a contravariantly, resp. covariantly, finite subcategory in $\mathfrak{C}^{2}$. We call the factor categories $\mathcal{A}(\mathfrak{C})=\mathfrak{C}^{2} / \mathfrak{Y}$ and $\mathcal{B}(\mathfrak{C})=\mathfrak{C}^{2} / \mathfrak{X}$, the Freyd categories of $\mathfrak{C}$. The category $\mathcal{A}(\mathfrak{C})$ is obtained by killing the ideal of morphisms of $\mathfrak{C}^{2}$ which factorize through split monics and the category $\mathcal{B}(\mathfrak{C})$ is obtained by killing the ideal of morphisms of $\mathfrak{C}^{2}$ which factorize through split epics. These categories occur in the literature for the first time in the work of Gabriel in the late fifties, see [23], [24]. Freyd used these categories systematically in his study of the representation of an additive category, for instance the stable homotopy category of finite $\mathrm{CW}$-complexes, into an additive category with a more rich and rigid structure, via nicely behaved embeddings, see [20], [21]. Since then the Freyd categories have found important applications and they are omnipresent in representation theory, in stable homotopy theory and homological algebra, see for instance $[\mathbf{2 2}]$, where the objects of a Freyd category appear as the values of an $L^{2}$-cohomology theory defined on Hilbert modules over Von Neumann algebras.

Received 28 January 2000, revised 28 November 2000; published on 27 December 2000.

2000 Mathematics Subject Classification: Primary 18E10, 18E30, 16D90; Secondary 16G10, 16G60, 16L60

Key words and phrases: Contravariantly Finite and Reflective Subcategories, Freyd and Auslander Categories, Abelian, Weak Abelian and Triangulated Categories, Pure-Semisimple Categories, Flat and Homological Functors, Ind-objects and Pro-objects.

(C) 2000, Apostolos Beligiannis. Permission to copy for private use granted. 
It is easy to see that $\mathcal{B}(\mathfrak{C})$ is dual to $\mathcal{A}\left(\mathfrak{C}^{o p}\right)$, so it is enough to study only $\mathcal{A}(\mathfrak{C})$. The main property of the Freyd category $\mathcal{A}(\mathfrak{C})$ is deduced from the fact that $\mathcal{A}(\mathfrak{C})$ is a special kind of a right triangulated [10] or suspended [30] category: the triangulation of $\mathcal{A}(\mathfrak{C})$ is induced by a right homotopy pair (we refer to section 2 for definitions). For this reason we prove in section 2 a result which constructs in a universal way left, resp. right, triangulated categories from given left, resp. right, homotopy pairs.

The paper roughly consists of two parts. In the first part (sections 2 to 6 ) we study the basic properties of the Freyd categories and in the second part (sections 7,8 ) we give applications to module theory. More presicely in section 3 we obtain the Freyd categories as right or left triangulated categories induced by suitable right or left homotopy pairs. The Freyd category $\mathcal{A}(\mathfrak{C})$ admits a nice description as the category mod-C $\mathfrak{C}$ of contravariant finitely presented additive functors from $\mathfrak{C}$ to the category $\mathfrak{A} b$ of abelian groups and $\mathcal{B}(\mathfrak{C})$ is equivalent to the dual of the category $\mathfrak{C}-\bmod$ of covariant finitely presented functors $\mathfrak{C} \rightarrow \mathfrak{A} b$. We prove that $\mathcal{A}(\mathfrak{C})$, resp. $\mathcal{B}(\mathfrak{C})$, is the reflection of $\mathfrak{C}$ in the category of additive categories with cokernels, resp. kernels, and cokernel, resp. kernel, preserving functors. As a consequence the last categories are tripleable over the category of additive categories.

By a result of Freyd [20] (which goes back to H. Cartan), the category $\mathcal{A}(\mathfrak{C})$ is abelian iff $\mathfrak{C}$ is right coherent, that is any morphism in $\mathfrak{C}$ admits a weak kernel. In section 4 we study other homological consequences of the coherence of $\mathfrak{C}$, in particular we characterize when $\mathcal{A}(\mathfrak{C})$ is a module category. In section 5 using the universal properties of the Freyd categories $\mathcal{A}(\mathfrak{C}), \mathcal{B}(\mathfrak{C})$ we construct a tensor product $-\otimes_{\mathfrak{C}}-: \mathcal{A}(\mathfrak{C}) \times \mathcal{B}(\mathfrak{C})^{o p} \rightarrow \mathfrak{A} b$ which, in case $\mathfrak{C}$ is skeletally small, is the restriction of the usual tensor product between right and left $\mathfrak{C}$-modules. The main result of this section is that for any coherent category $\mathfrak{C}$ the abelian Freyd categories $\mathcal{A}(\mathfrak{C}), \mathcal{B}(\mathfrak{C})$ have the same global dimension. In addition we prove that the classical formula of Auslander $[4]$ is valid in this setting.

In section 6 we construct in a simple way the free abelian category of an arbitrary category. The free abelian category of the additive category $\mathfrak{C}$ is the iterated Freyd category $\mathcal{A B}(\mathfrak{C})$. If $\mathfrak{C}$ is small the first construction of the free abelian category is due to Freyd [20]. Later Addelman [1] gave a more simple but still complicated construction. In case $\mathfrak{C}$ is skeletally small, an equivalent formulation of the fact that $\mathcal{A B}(\mathfrak{C})$ is the free abelian category of $\mathfrak{C}$ was given independently by Krause $[\mathbf{3 1}]$. We characterize the free abelian categories as the Auslander categories, i.e. as the abelian categories $\mathcal{H}$ with enough projectives and injectives, having global dimension gl.dim $\mathcal{H} \leqslant 2$ and dominant dimension dom.dim $\mathcal{H} \geqslant 2$. Hence we can associate to any category its Auslander category. In this setting we generalize the well-known correspondence between Auslander algebras and representation finite Artin algebras [3], to abelian categories.

Section 7 is devoted to the study of modules over a skeletally small additive category $\mathfrak{C}$, using as main tools the concepts of contavariantly and covariantly finite subcategories and the Freyd categories of $\mathfrak{C}$. We denote by Mod-C the category of right $\mathfrak{C}$-modules with objects the contravariant additive functors $\mathfrak{C} \rightarrow \mathfrak{A} b$. First we give necessary and sufficient conditions for the full subcategories Flat $(\operatorname{Mod}-\mathfrak{C})$, resp. Proj(Mod-C $)$, of flat, resp. projective, modules to be covariantly, resp. reflective, finite subcategories and similarly for the full subcategories FPInj(Mod-C $)$, resp. Inj(Mod-C $)$, of FP-injective, resp. injective, modules to be contravariantly finite, resp. coreflective, subcategories of Mod-C $\mathfrak{C}$. The main result of this section characterizes when the categories Flat $(\operatorname{Mod}-\mathfrak{C})$ and $\operatorname{Proj}(\operatorname{Mod}-\mathfrak{C})$ are abelian (Grothendieck) categories, answering a question of Simson [43]. In particular we show that the category Proj(Mod- $\mathfrak{C})$ is abelian iff the module category Mod- $\mathfrak{C}$ is a perfect Auslander category. As a corollary we deduce that $\operatorname{Mod}-\mathfrak{C}$ is pure semisimple $[43]$ iff $\operatorname{Proj}(\operatorname{Mod}-\mathcal{A}(\mathfrak{C}))$ is an abelian category iff $\operatorname{Mod}-\mathcal{A}(\mathfrak{C})$ is an Auslander category over a left coherent category iff $\operatorname{Mod}-\mathcal{A}(\mathfrak{C})$ is the Freyd category $\mathcal{A}(\operatorname{Mod}-\mathfrak{C})$. For any additive category $\mathfrak{C}$, we denote by $\operatorname{Ind}(\mathfrak{C}), \operatorname{Pro}(\mathfrak{C})$, the associated categories of Ind-objects, Pro-objects $[\mathbf{2 6}],[\mathbf{4 5}]$. We give necessary and sufficient conditions for the abelianness of $\mathcal{I} n d(\mathfrak{C}), \operatorname{Pro}(\mathfrak{C})$, generalizing results of 
Stauffer $[\mathbf{4 5}]$. If $\mathfrak{C}$ is abelian we have as a corollary the classical result that the full subcategory Lex $\left(\mathfrak{C}^{o p}, \mathfrak{A} b\right)$, resp. Rex $\left(\mathfrak{C}^{o p}, \mathfrak{A} b\right)$, of contravariant left, resp. right, exact functors, is a Giraud, resp. co-Giraud, subcategory of Mod-C $\mathfrak{C}$. Finally we prove that for a coherent category $\mathfrak{C}$, the category Flat (Mod- $\mathfrak{C})$ is abelian iff the category Flat $(\mathfrak{C}-$ Mod) is abelian iff $\mathfrak{C}$ is abelian. If in addition any left and right flat $\mathfrak{C}$-module is projective then Mod- $\mathfrak{C}$ is Auslander iff $\mathfrak{C}-$ Mod is Auslander, hence in this case the Auslander condition is symmetric.

In section 8 we study weak abelian categories, a class of categories which contains the triangulated categories. A weak abelian category, which can be thought of as an internal version of a triangulated category, is a coherent category in which any morphism is a weak kernel and a weak cokernel, see $[\mathbf{2 0}]$. We characterize the weak abelian categories as the categories $\mathfrak{C}$ for which any left and right injective $\mathfrak{C}$-module is flat or equivalently as the full subcategories of projective-injective objects of Frobenius abelian categories. Using this result we deduce that the module category Mod- $\mathfrak{C}$ is Frobenius iff $\mathfrak{C}$ is weak abelian and $\mathcal{A}(\mathfrak{C})$ or $\mathcal{B}(\mathfrak{C})$ is Noetherian. In case $\mathfrak{C}$ is a left, resp. right, triangulated category, the Freyd category $\mathcal{A}(\mathfrak{C})$, resp. $\mathcal{B}(\mathfrak{C})$, is the free "homological" category of $\mathfrak{C}$. If $\mathfrak{C}$ is triangulated or more generally weak abelian, then $\mathfrak{C}$ enjoys the nice property that its Freyd categories $\mathcal{A}(\mathfrak{C})$ and $\mathcal{B}(\mathfrak{C})$ are equivalent. The main result of this section shows that if $\mathfrak{C}$ is a weak abelian category such that its Auslander category is Noetherian (Artinian), then the category of Ind-objects (Pro-objects) of the stable category $\mathcal{A}(\mathfrak{C})$ of the Freyd category $\mathcal{A}(\mathfrak{C})$ modulo projectives or injectives, is a triangulated category. This generalizes a recent result of Krause [33].

Some of the results of this paper are known in some version. We believe that the presentation is new and more simple and conceptual. A fixed convention through the paper is that the composition of morphisms in a given category is meant in the diagrammatic order: the composition of $f: A \rightarrow B$ and $g: B \rightarrow C$ is denoted by $f \circ g$.

\section{Homotopy Pairs and Triangulations}

Throughout this section we fix a pair $(\mathfrak{C}, \mathfrak{X})$ consisting of an additive category $\mathfrak{C}$ and a full additive subcategory $\mathfrak{X} \subseteq \mathfrak{C}$ of $\mathfrak{C}$, closed under direct summands and isomorphisms.

A morphism $f: A \rightarrow B$ in $\mathfrak{C}$ is called an $\mathfrak{X}$-epic if the induced morphism $\mathfrak{C}(X, f)$ : $\mathfrak{C}(X, A) \rightarrow \mathfrak{C}(X, B)$ in $\mathfrak{A} b$ is an epimorphism, $\forall X \in \mathfrak{X}$. We denote by $\mathcal{E}^{\mathfrak{X}}$ the full subcategory of the morphism category $\mathfrak{C}^{2}$, consisting of all $\mathfrak{X}$-epics. The subcategory $\mathfrak{X}$ is called contravariantly finite $[\mathbf{9}]$ iff $\forall A \in \mathfrak{C}$, there exists a morphism $\chi_{A}: X_{A} \rightarrow A$ in $\mathcal{E}^{\mathfrak{X}}$, with $X_{A} \in \mathfrak{X}$; the morphism $\chi_{A}$ is called a right $\mathfrak{X}$-approximation of $A$. Dually we define $\mathfrak{X}$-monics, covariant finiteness of $\mathfrak{X}$, and left $\mathfrak{X}$-approximations. The full subcategory of $\mathfrak{C}^{2}$ consisting of all $\mathfrak{X}$-monics is denoted by $\mathcal{M}^{\mathfrak{X}}$.

Definition 2.1. The pair $(\mathfrak{C}, \mathfrak{X})$ is called a left homotopy pair, if $\mathfrak{X}$ is contravariantly finite in $\mathfrak{C}$ and any $\mathfrak{X}$-epic has a kernel in $\mathfrak{C}$. The pair $(\mathfrak{C}, \mathfrak{X})$ is called a right homotopy pair if $\mathfrak{X}$ is covariantly finite in $\mathfrak{C}$ and any $\mathfrak{X}$-monic has a cokernel in $\mathfrak{C}$. A left and right homotopy pairs is called a homotopy pair.

If $(\mathfrak{C}, \mathfrak{X})$ is a left homotopy pair, we denote by lex $(\mathfrak{X})$ the category of diagrams of the form

$A \stackrel{g}{\rightarrow} B \stackrel{f}{\rightarrow} C$, where $f \in \mathcal{E}^{\mathfrak{X}}$ and $g=\operatorname{ker}(f)$. It is easy to see that $\operatorname{lex}(\mathfrak{X}) \approx \mathcal{E}^{\mathfrak{X}}$. Dually we define the category $\operatorname{rex}(\mathfrak{X})$ and the equivalence $\operatorname{rex}(\mathfrak{X}) \approx \mathcal{M}^{\mathfrak{X}}$, if $(\mathfrak{C}, \mathfrak{X})$ is right homotopy pair. For any pair $(\mathfrak{C}, \mathfrak{X})$ we consider the induced stable category $\mathfrak{C} / \mathfrak{X}$, which is the factor category of $\mathfrak{C}$ modulo the ideal of morphisms which factor through $\mathfrak{X}$. We denote by $\varpi_{\mathfrak{X}}: \mathfrak{C} \rightarrow \mathfrak{C} / \mathfrak{X}$ the natural functor, and we set $\varpi_{\mathfrak{X}}(A)=\underline{A}$ and $\varpi_{\mathfrak{X}}(f)=\underline{f}$.

By $[\mathbf{1 0}]$ any left, resp. right, homotopy pair $(\mathfrak{C}, \mathfrak{X})$ induces a left, resp. right, triangulated structure on the stable category $\mathfrak{C} / \mathfrak{X}$. For the notion of a (left or right) triangulated category we refer to $[\mathbf{1 0}],[\mathbf{3 0}],[\mathbf{5 0}]$. We note only that a left, resp. right, triangulated category is a triple $(\mathfrak{C}, \Omega, \Delta)$, resp. $(\mathfrak{C}, \Sigma, \nabla)$ where $\mathfrak{C}$ is an additive category, $\Omega: \mathfrak{C} \rightarrow \mathfrak{C}$, resp. $\Sigma: \mathfrak{C} \rightarrow \mathfrak{C}$, is an 
additive functor and $\Delta$, resp. $\nabla$, is a class of diagrams in $\mathfrak{C}$ of the form $\Omega(C) \rightarrow A \rightarrow B \rightarrow C$, resp. $A \rightarrow B \rightarrow C \rightarrow \Sigma(A)$, satisfying certain axioms (see [10], [30] for details). The diagrams in $\Delta$, resp. $\nabla$, are called triangles. In case $\Omega$, resp. $\Sigma$, is an equivalence, $(\mathfrak{C}, \Omega, \Delta)$, resp. $(\mathfrak{C}, \Sigma, \nabla)$ is called a triangulated category [50], [51].

The aim of this section is to complete the main result of [10], by characterizing the left triangulated category induced from a left homotopy pair, by a universal property. To this end we need some definitions. Consider a pair $(\mathfrak{C}, \mathfrak{X})$ with $\mathfrak{X}$ contravariantly finite in $\mathfrak{C}$. We denote by $\mathrm{e}_{i}: \operatorname{lex}(\mathfrak{X}) \rightarrow \mathfrak{C}, i=1,2,3$ the obvious functors $\mathrm{e}_{i}\left(A_{3} \rightarrow A_{2} \rightarrow A_{1}\right)=A_{i}$. A $\vartheta$-functor $(F, \Theta): \mathfrak{C} \rightarrow(\mathfrak{D}, \Omega, \Delta)$ to a left triangulated category $\mathfrak{D}$, is a pair consisting of a functor $F: \mathfrak{C} \rightarrow \mathfrak{D}$ and a functor $\Theta: \operatorname{lex}(\mathfrak{X}) \rightarrow \mathfrak{D}^{2}$, where $\Theta(E)=\vartheta_{E} \in \mathfrak{D}\left[\Omega F\left(e_{1}(E)\right), F\left(e_{3}(E)\right)\right]$, $\forall E \in \operatorname{lex}(\mathfrak{X})$, such that: if $E=A_{3} \stackrel{g}{\rightarrow} A_{2} \stackrel{f}{\rightarrow} A_{1} \in \operatorname{lex}(\mathfrak{X})$, then:

$$
\Omega F\left(A_{1}\right) \stackrel{\vartheta_{E}}{\longrightarrow} F\left(A_{3}\right) \stackrel{F g}{\longrightarrow} F\left(A_{2}\right) \stackrel{F f}{\longrightarrow} F\left(A_{1}\right) \in \Delta
$$

Let $(\mathfrak{C}, \mathfrak{X})$ be a left homotopy pair. Then the loop functor $\Omega_{\mathfrak{X}}: \mathfrak{C} / \mathfrak{X} \rightarrow \mathfrak{C} / \mathfrak{X}$ is defined as follows: if $A \in \mathfrak{C}$, then consider the object $K_{A} \stackrel{k_{A}}{\longrightarrow} X_{A} \stackrel{\chi_{A}}{\longrightarrow} A \in \operatorname{lex}(\mathfrak{X})$, and set $\Omega_{\mathfrak{X}}(A)=\underline{K}_{A}$. If $f: A \rightarrow B$ is a morphism in $\mathfrak{C}$, then $\Omega_{\mathfrak{X}}(\underline{f})=\underline{k}_{f}$, where $k_{f}: K_{A} \rightarrow K_{B}$ is the unique morphism such that: $k_{A} \circ x_{f}=k_{f} \circ k_{B}$, where $x_{f}: X_{A} \rightarrow X_{B}$ is an arbitrary morphism with $x_{f} \circ \chi_{B}=\chi_{A} \circ f$. The morphism $x_{f}$ exists, since $\chi_{B}$ is a right $\mathfrak{X}$-approximation of $B$. It is shown in [10] that $\Omega_{\mathfrak{X}}$ is a well defined additive functor.

Note that by [11], if $\mathfrak{X}$ is in addition covariantly finite and any left $\mathfrak{X}$-approximation has a cokernel in $\mathfrak{C}$, then the loop functor $\Omega_{\mathfrak{X}}$ admits a left adjoint, the suspension functor $\Sigma_{\mathfrak{X}}$. In particular if $(\mathfrak{C}, \mathfrak{X})$ is a homotopy pair, then $\left(\Sigma_{\mathfrak{X}}, \Omega_{\mathfrak{X}}\right)$ is an adjoint pair in $\mathfrak{C} / \mathfrak{X}$.

Suppose now $E=A \stackrel{g}{\rightarrow} B \stackrel{f}{\rightarrow} C \in \operatorname{lex}(\mathfrak{X})$; then the characteristic class $\operatorname{ch}(E)$ of $E$ is defined as follows; consider the object $K_{A} \stackrel{k_{A}}{\longrightarrow} X_{A} \stackrel{\chi_{A}}{\longrightarrow} A \in \operatorname{lex}(\mathfrak{X})$. Since $X_{A} \in \mathfrak{X}$ and $f \in \mathcal{E}^{\mathfrak{X}}$, there exists $\delta: X_{A} \rightarrow A B$, with: $\delta \circ f=\chi_{A}$. Hence there exists a unique morphism $\gamma: K_{A} \rightarrow C$, with $\gamma \circ g=k_{A} \circ \delta$. We define $\operatorname{ch}(E)=\underline{\gamma}$; it is shown in $[\mathbf{1 0}]$ that $\operatorname{ch}(E): \Omega_{\mathfrak{X}}(\underline{A}) \rightarrow \underline{C}$ is independent of the choice of $\delta$. Finally we define $\Delta_{\mathfrak{X}}$ to be the class of diagrams $\Omega_{\mathfrak{X}}\left(\underline{C}^{\prime}\right) \rightarrow \underline{A}^{\prime} \rightarrow \underline{B}^{\prime} \rightarrow \underline{C}^{\prime}$ in $\mathfrak{C} / \mathfrak{X}$ which are isomorphic to diagrams of the form

$$
\Omega_{\mathfrak{X}}(\underline{C}) \stackrel{\operatorname{ch}(E)}{\longrightarrow} \underline{A} \stackrel{\underline{g}}{\longrightarrow} \underline{B} \stackrel{\stackrel{f}{\longrightarrow}}{\longrightarrow}
$$

for some object $E=A \stackrel{g}{\rightarrow} B \stackrel{f}{\rightarrow} C \in \operatorname{lex}(\mathfrak{X})$.

Theorem 2.2. Let $(\mathfrak{C}, \mathfrak{X})$ be a left homotopy pair. Then there exists a left triangulated structure $\left(\mathfrak{C} / \mathfrak{X}, \Omega_{\mathfrak{X}}, \Delta_{\mathfrak{X}}\right)$ on the stable category $\mathfrak{C} / \mathfrak{X}$, and a $\vartheta$-functor $\left(\varpi_{\mathfrak{X}}, \Theta\right): \mathfrak{C} \rightarrow\left(\mathfrak{C} / \mathfrak{X}, \Omega_{\mathfrak{X}}, \Delta_{\mathfrak{X}}\right)$, satisfying the following universal property:

$(\dagger)$ If $(F, \Phi): \mathfrak{C} \rightarrow \mathfrak{D}$ is a $\vartheta$-functor to a left triangulated category $(\mathfrak{D}, \Omega, \Delta)$ such that $F(\mathfrak{X})=0$, then there exists a unique exact functor of left triangulated categories $F^{!}$: $\mathfrak{C} / \mathfrak{X} \rightarrow \mathfrak{D}$, such that: $F^{!} \varpi_{\mathfrak{X}}=F$.

Proof. For the first part see [10]. Define $\Theta: \operatorname{lex}(\mathfrak{X}) \rightarrow(\mathfrak{C} / \mathfrak{X})^{2}$ as follows: if $E=A \stackrel{g}{\rightarrow} B \stackrel{f}{\rightarrow}$ $C \in \operatorname{lex}(\mathfrak{X})$, then $\Theta(E)=\operatorname{ch}(E)$, and if $(a, b, c): E_{1} \rightarrow E_{2}$ is a morphism in lex $(\mathfrak{X})$, then $\Theta(a, b, c)=\left(\Omega_{\mathfrak{X}}(\underline{b}), \underline{a}\right): \operatorname{ch}\left(E_{1}\right) \rightarrow \operatorname{ch}\left(E_{2}\right):$

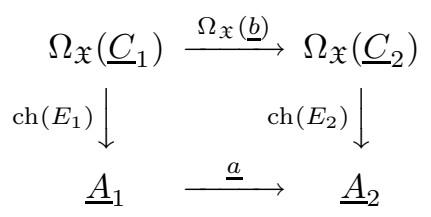

It is easy to see that the above diagram commutes and the pair $\left(\varpi_{\mathfrak{X}}, \Theta\right)$ is a well defined $\vartheta$-functor. Consider now a left triangulated category $(\mathfrak{D}, \Omega, \Delta)$, and let $(F, \Phi): \mathfrak{C} \rightarrow(\mathfrak{D}, \Omega, \Delta)$ 
be a $\vartheta$-functor such that $F(\mathfrak{X})=0$. The last condition implies that there exists a unique functor $F^{!}: \mathfrak{C} / \mathfrak{X} \rightarrow \mathfrak{D}$, such that: $F^{!} \varpi_{\mathfrak{X}}=F$. We have to show that $F^{!}$is an exact functor between left triangulated categories. For any $A \in \mathfrak{C}$, let $E_{A}=K_{A} \stackrel{k_{A}}{\longrightarrow} X_{A} \stackrel{\chi_{A}}{\longrightarrow} A$ be in lex $(\mathfrak{X})$. Since $(F, \Phi)$ is a $\vartheta$-functor and $F(\mathfrak{X})=0$, the diagram $0 \rightarrow T F(A) \stackrel{\phi_{E_{A}}}{\longrightarrow} F\left(K_{A}\right) \rightarrow 0$ is a triangle in $\Delta$, where $\phi_{E_{A}}=\Phi\left(E_{A}\right)$. Since $F^{!} \varpi_{\mathfrak{X}}=F$ and $\varpi_{\mathfrak{X}}\left(K_{A}\right)=\Omega_{\mathfrak{X}}(\underline{A})$, it follows directly that $\phi_{E_{A}}$ is invertible and $\phi_{E}: \Omega F^{!} \rightarrow F^{!} \Omega_{\mathfrak{X}}$ is a natural isomorphism. It remains to show that $F^{!}$maps $\Delta_{\mathfrak{X}}$ to $\Delta$. Let $\Omega_{\mathfrak{X}}(\underline{C}) \stackrel{\operatorname{ch}(E)}{\longrightarrow}(\underline{A}) \stackrel{g}{\rightarrow} \underline{B} \stackrel{\stackrel{f}{\longrightarrow}}{\longrightarrow} \underline{C}$ be a triangle in $\Delta_{\mathfrak{X}}$ induced from the object $E=A \stackrel{g}{\rightarrow} B \stackrel{f}{\rightarrow} C \in \operatorname{lex}(\mathfrak{X})$. Then we have a triangle:

$$
\Omega F^{!}(\underline{C}) \stackrel{\phi_{E}}{\longrightarrow} F^{!}(\underline{A}) \stackrel{F^{!}(\underline{g})}{\longrightarrow} F^{!}(\underline{B}) \stackrel{F^{!}(\underline{f})}{\longrightarrow} F^{!}(\underline{C}) \in \Delta
$$

Consider the object $E_{C}=K_{C} \stackrel{k_{C}}{\longrightarrow} X_{C} \stackrel{\chi_{C}}{\longrightarrow} C \in \operatorname{lex}(\mathfrak{X})$ induced from the right $\mathfrak{X}$-approximation of $C$. Then we have a morphism $E_{C} \rightarrow E$ in lex $(\mathfrak{X})$ :

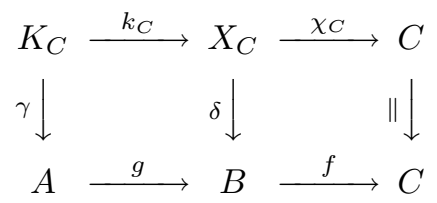

where $\underline{\gamma}=\operatorname{ch}(E)$. Since $(F, \Phi)$ is a $\vartheta$-functor, the above diagram implies that $\phi_{E_{C}} \circ F^{!}(\operatorname{ch}(E))$ $=\phi_{E}$. Since $\phi_{E_{C}}$ is invertible, the diagram $T F^{!}(\underline{C}) \stackrel{\phi_{E}}{\longrightarrow} F^{!}(\underline{A}) \stackrel{F^{!}(\underline{g})}{\longrightarrow} F^{!}(\underline{B}) \stackrel{F^{!}(\underline{f})}{\longrightarrow} F^{!}(\underline{C})$ is isomorphic to the triangle $\Omega F^{!}(\underline{C}) \stackrel{\phi_{E_{C}}}{\longrightarrow} F^{!}(\underline{A}) \stackrel{F^{!}(\underline{g})}{\longrightarrow} F^{!}(\underline{B}) \stackrel{F^{!}(\underline{f})}{\longrightarrow} F^{!}(\underline{C})$ in $\Delta$, thus it is a triangle. Hence $F^{!}$is exact. The uniqueness of $F^{!}$is trivial.

Note that Theorem 2.2 and its dual, covers most of the known cases for which a stable category is left or right triangulated. If $(\mathfrak{C}, \mathfrak{X}),(\mathfrak{D}, \mathfrak{Y})$ are left homotopy pairs, then $F: \mathfrak{C} \rightarrow \mathfrak{D}$ is a homotopy functor, if $F(\operatorname{lex}(\mathfrak{X})) \subseteq \operatorname{lex}(\mathfrak{Y})$ and $F(\mathfrak{X}) \subseteq \mathfrak{Y}$. Trivially we have the following.

Corollary 2.3. If $F:(\mathfrak{C}, \mathfrak{X}) \rightarrow(\mathfrak{D}, \mathfrak{Y})$ is a homotopy functor between left homotopy pairs, then the induced functor $\underline{F}:\left(\mathfrak{C} / \mathfrak{X}, \Omega_{\mathfrak{X}}, \Delta_{\mathfrak{X}}\right) \rightarrow\left(\mathfrak{D} / \mathfrak{Y}, \Omega_{\mathfrak{Y}}, \Delta_{\mathfrak{Y}}\right)$ is exact.

We refer to $[\mathbf{1 3}]$ for an interpretation of the stable category $\mathfrak{C} / \mathfrak{X}$ as the homotopy category of a co-Waldhausen, resp. Quillen closed model, category structure on $\mathfrak{C}$, if $(\mathfrak{C}, \mathfrak{X})$ is a left homotopy pair, resp. homotopy pair.

\section{The Freyd Categories}

Throughout this section we fix an additive category $\mathfrak{C}$ with split idempotents.

In the morphism category $\mathfrak{C}^{2}$, we denote an object $f: A \rightarrow B$ by $(A, f, B)$, and a morphism by $(a, b):(A, f, B) \rightarrow(C, g, D)$, i.e. $a: A \rightarrow C, b: B \rightarrow D$ with $a \circ g=f \circ b$. In $\mathfrak{C}^{2}$ we consider the full subcategories, $\mathfrak{X}$ consisting of all split monics, and $\mathfrak{Y}$ consisting of all split epics. Now $\mathfrak{X}$ is contravariantly finite in $\mathfrak{C}^{2}$ with right $\mathfrak{X}$-approximation of $f: A \rightarrow B$ the morphism $\left(1_{A},{ }^{t}\left(f, 1_{B}\right)\right):\left(A,\left(1_{A}, 0\right), A \oplus B\right) \rightarrow(A, f, B)$. Similarly $\mathfrak{Y}$ is covariantly finite. The morphism $(a, b):(A, f, B) \rightarrow(C, g, D)$ is $\mathfrak{X}$-epic iff $a, b$ are split epics, and is $\mathfrak{Y}$-monic iff $a, b$ are split monics. Since in $\mathfrak{C}$ idempotents split, split epics have kernels and split monics have cokernels, and the same is true in $\mathfrak{C}^{2}$. Hence in $\mathfrak{C}^{2}$ any $\mathfrak{X}$-epic has a kernel and any $\mathfrak{Y}$-monic has a cokernel. Thus $\left(\mathfrak{C}^{2}, \mathfrak{X}\right)$ is a left homotopy pair and $\left(\mathfrak{C}^{2}, \mathfrak{Y}\right)$ is a right homotopy pair. By Theorem 2.2 the stable category $\mathfrak{C}^{2} / \mathfrak{X}$ is left triangulated, and the stable category $\mathfrak{C}^{2} / \mathfrak{Y}$ is right triangulated. By construction the loop, resp. suspension, functor in $\mathfrak{C}^{2} / \mathfrak{X}$, resp. in $\mathfrak{C}^{2} / \mathfrak{Y}$, is zero. For example the kernel of the right $\mathfrak{X}$-approximation $\left(1_{A},{ }^{t}\left(f, 1_{B}\right)\right):\left(A,\left(1_{A}, 0\right), A \oplus B\right) \rightarrow$ $(A, f, B)$ is the object $(0,0, A) \in \mathfrak{X}$. 
Definition 3.1. The Freyd categories of $\mathfrak{C}$ are the stable categories

$$
\mathcal{A}(\mathfrak{C}):=\mathfrak{C}^{2} / \mathfrak{Y}, \quad \mathcal{B}(\mathfrak{C}):=\mathfrak{C}^{2} / \mathfrak{X} .
$$

We denote by $\{A, f, B\}$ the object $(A, f, B) \in \mathfrak{C}^{2}$, when considered as an object in $\mathcal{A}(\mathfrak{C})$, and by $[A, f, B]$ when considered as an object in $\mathcal{B}(\mathfrak{C})$. Similarly we denote by $\{a, b\}$ the morphism $(a, b)$ when considered as a morphism in $\mathcal{A}(\mathfrak{C})$, and by $[a, b]$ when considered as a morphism in $\mathcal{B}(\mathfrak{C})$. It is easy to see that if $(a, b):(A, f, B) \rightarrow(C, g, D)$ is a morphism in $\mathfrak{C}^{2}$, then $\{a, b\}=0$ in $\mathcal{A}(\mathfrak{C})$ iff $\exists h: B \rightarrow C$ with $h \circ g=b$, and $[a, b]=0$ in $\mathcal{B}(\mathfrak{C})$ iff $\exists h: B \rightarrow C$ with $f \circ h=a$. In particular $\{A, f, B\}=0$ iff $f$ is split epic, and $[A, f, B]=0$ iff $f$ is split monic. Hence $\mathcal{A}(\mathfrak{C}), \mathcal{B}(\mathfrak{C})$ are the homotopy categories introduced by Freyd in $[\mathbf{2 0}]$. The category $\mathfrak{C}$ is fully imbedded in its Freyd categories $\mathcal{A}(\mathfrak{C}), \mathcal{B}(\mathfrak{C})$ by the functors:

$$
\mathbf{P}_{\mathfrak{C}}: \mathfrak{C} \rightarrow \mathcal{A}(\mathfrak{C}), \quad \mathbf{Q}_{\mathfrak{C}}: \mathfrak{C} \rightarrow \mathcal{B}(\mathfrak{C})
$$

defined as: $\mathbf{P}_{\mathfrak{C}}(A)=\{0,0, A\}, \mathbf{P}_{\mathfrak{C}}(a)=\{0, a\}$ and $\mathbf{Q}_{\mathfrak{C}}(A)=[A, 0,0], \mathbf{Q}_{\mathfrak{C}}(a)=[a, 0]$.

If there is no confusion we write $\mathbf{P}, \mathbf{Q}$ for $\mathbf{P}_{\mathfrak{C}}, \mathbf{Q}_{\mathfrak{C}}$. Let $\mathbf{I d e m}$ be the category of additive categories with split idempotents with morphisms (equivalence classes of) additive functors. We consider in Idem the subcategories Coker, resp. Ker, of additive categories with cokernels, resp. kernels, with morphisms (equivalence classes of) cokernel, resp. kernel, preserving functors.

Corollary 3.2. $\mathcal{A}(\mathfrak{C})$, resp. $\mathcal{B}(\mathfrak{C})$, is the reflection of $\mathfrak{C}$ in Coker, resp. Ker.

Proof. Since $\mathcal{A}(\mathfrak{C})$ is a right triangulated category with zero suspension functor, $\mathcal{A}(\mathfrak{C})$ is a category with cokernels. Let $F: \mathfrak{C} \rightarrow \mathfrak{D}$ be a functor to a category with cokernels. $\mathfrak{D}$ can be considered as a right triangulated category with zero suspension functor. Extend $F$ to a functor $F^{\circ}: \mathfrak{C}^{2} \rightarrow \mathfrak{D}$ by $F^{\circ}(A, f, B)=$ Coker $(f)$. Obviously $F^{\circ}(\mathfrak{Y})=0$. By the dual of Theorem 2.2 , there exists a unique exact functor $F^{!}: \mathcal{A}(\mathfrak{C}) \rightarrow \mathfrak{D}$ of right triangulated categories, with $F^{!} \varpi_{\mathfrak{Y}}=F^{\circ}$. Since exact functors between right triangulated categories with zero suspension functors are simply cokernel preserving functors, and since trivially $F^{!} \mathbf{P}=F$, we see that the functor $\mathbf{P}: \mathfrak{C} \rightarrow \mathcal{A}(\mathfrak{C})$ is the reflection of $\mathfrak{C}$ in Coker. Similarly for $\mathcal{B}(\mathfrak{C})$.

Thus we can consider the functors $\mathcal{A}:$ Idem $\rightarrow$ Coker and $\mathcal{B}:$ Idem $\rightarrow$ Ker, defined on morphisms by $\mathcal{A}(F)\{a, b\}=\{F a, F b\}, \mathcal{B}(F)[a, b]=[F a, F b]$, for an additive functor $F: \mathfrak{C} \rightarrow$ $\mathfrak{D}$. $\mathcal{A}$ is the left adjoint of the inclusion $\mathcal{I}:$ Coker $\hookrightarrow$ Idem, and $\mathcal{B}$ is the left adjoint of the inclusion $\mathcal{J}:$ Ker $\hookrightarrow$ Idem.

For any morphism $f: A \rightarrow B$ in $\mathfrak{C}$ we have the exact sequences:

$$
\begin{gathered}
\mathbf{P}(A) \stackrel{\mathbf{P}(f)}{\longrightarrow} \mathbf{P}(B) \stackrel{\left\{0,1_{B}\right\}}{\longrightarrow}\{A, f, B\} \longrightarrow 0 \\
0 \longrightarrow[A, f, B] \stackrel{\left[1_{A}, 0\right]}{\longrightarrow} \mathbf{Q}(A) \stackrel{\mathbf{Q}(f)}{\longrightarrow} \mathbf{Q}(B) .
\end{gathered}
$$

Indeed $\left\{0,1_{B}\right\}: \mathbf{P}(B) \rightarrow\{A, f, B\}$ is always epic since if $\left\{0,1_{B}\right\} \circ\{a, b\}=0$, where $\{a, b\}:\{A, f, B\} \rightarrow\{C, g, D\}$, then $\{a, b\}=0$ since $b$ factors through $g$. Now obviously $\mathbf{P}(f) \circ\left\{0,1_{B}\right\}=0$, and if $\mathbf{P}(f) \circ\{0, b\}=0$ for $\{0, b\}: \mathbf{P}(B) \rightarrow\{C, g, D\}$, then $f \circ b=a \circ g$ for a morphism $a: A \rightarrow C$. Then $\{a, b\}:\{A, f, B\} \rightarrow\{C, g, D\}$ satisfies $\left\{0,1_{B}\right\} \circ\{a, b\}=\{0, b\}$. Hence $\left\{0,1_{B}\right\}=\operatorname{coker} \mathbf{P}(f)$. Similarly for $\mathcal{B}(\mathfrak{C})$.

Lemma 3.3. The functor $\mathbf{P}_{\mathfrak{C}}: \mathfrak{C} \rightarrow \mathcal{A}(\mathfrak{C})$, resp. $\mathbf{Q}_{\mathfrak{C}}: \mathfrak{C} \rightarrow \mathcal{B}(\mathfrak{C})$, preserves kernels, resp. cokernels, and has a left, resp. right, adjoint $\Phi_{\mathfrak{C}}$, resp. $\Psi_{\mathfrak{C}}$, iff $\mathfrak{C}$ has cokernels, resp. kernels.

Proof. Let $g: C \rightarrow A$ be a kernel of $f: A \rightarrow B$, and let $\{0, b\}:\{X, h, Y\} \rightarrow \mathbf{P}(A)$ be such that $\{0, b\} \circ \mathbf{P}(f)=0$. Then $b \circ f=0$ and $h \circ b=0$. Hence $b=a \circ g$ for a unique morphism $a: Y \rightarrow C$, and $h \circ a \circ g=0 \Rightarrow h \circ a=0$, because $g$ is monic. Then $\{0, a\}:\{X, h, Y\} \rightarrow \mathbf{P}(C)$ is a morphism with $\{0, a\} \circ \mathbf{P}(g)=\{0, b\}$, and is unique with this property, since if $\{0, c\}$ is 
another such morphism, then $\{0, a \circ g-c \circ g\}:\{X, h, Y\} \rightarrow \mathbf{P}(A)$ is zero $\Rightarrow a \circ g-c \circ g$ factors through $0 \rightarrow A$, and this implies that $a=c$, since $g$ is monic. So $\mathbf{P}(g)=k e r \mathbf{P}(f)$. If $\mathfrak{C}$ has cokernels, then defining $\Phi\{A, f, B\}=\operatorname{Coker}(f)$, we see that $(\Phi, \mathbf{P})$ is an adjoint pair. Conversely if $\Phi$ exists, then since $\mathcal{A}(\mathfrak{C})$ has and $\Phi$ preserves cokernels, applying $\Phi$ to the exact sequence (1) and noting that $\Phi \mathbf{P}=\operatorname{Id}_{\mathfrak{C}}$, since $\mathbf{P}$ is fully faithful, we have that the cokernel of $f: A \rightarrow B$ is $\Phi\{A, f, B\}$. Dually for $\mathcal{B}(\mathfrak{C})$.

Theorem 3.4. (1) Consider the triple $\mathcal{T}$ generated by the adjoint pair $(\mathcal{A}, \mathcal{I})$, where $\mathcal{I}$ : Coker $\hookrightarrow$ Idem is the inclusion. Then: (i) $(\mathfrak{C}, F)$ is a $\mathcal{T}$-algebra iff $\mathfrak{C}$ has cokernels and $F=\Phi ;($ ii $) \quad R:(\mathfrak{C}, F) \rightarrow(\mathfrak{D}, G)$ is a morphism of $\mathcal{T}$-algebras iff $R$ preserves cokernels. The inclusion $\mathcal{I}$ is tripleable.

(2) Consider the triple $\mathcal{T}^{\prime}$ generated by the adjoint pair $(\mathcal{B}, \mathcal{J})$, where $\mathcal{J}: \operatorname{Ker} \hookrightarrow$ Idem is the inclusion. Then: (i) $(\mathfrak{C}, F)$ is a $\mathcal{T}^{\prime}$-algebra iff $\mathfrak{C}$ has kernels and $F=\Psi$; (ii) $S:(\mathfrak{C}, F) \rightarrow$ $(\mathfrak{D}, G)$ is a morphism of $\mathcal{T}^{\prime}$-algebras iff $S$ preserves kernels. The inclusion $\mathcal{J}$ is tripleable.

Proof. We prove the case of $\mathcal{B}(\mathfrak{C})$. We denote by $\mu=\mathcal{B}(\mathcal{B}(\mathfrak{C})) \rightarrow \mathcal{B}(\mathfrak{C}), \delta: \operatorname{Id}_{\text {Idem }} \rightarrow$ $\mathcal{B}$ the multiplication and the unit respectively, of the triple generated by the adjoint pair $(\mathcal{B}, \mathcal{J}) ; \mu$ is given by $\mu_{\mathfrak{C}}=\mathcal{J} \Psi_{\mathcal{B}(\mathfrak{C})}$, where $\Psi_{\mathcal{B}(\mathfrak{C})}$ is the right adjoint of the full imbed$\operatorname{ding} \mathbf{Q}_{\mathcal{B}(\mathfrak{C})}: \mathcal{B}(\mathfrak{C}) \rightarrow \mathcal{B}(\mathcal{B}(\mathfrak{C}))$, which exists since $\mathcal{B}(\mathfrak{C})$ has kernels. The unit $\delta$ is given by $\delta=\mathbf{Q}_{\mathfrak{C}}$. Suppose first that $\mathfrak{C}$ has kernels. Then $\Psi_{\mathfrak{C}}$ exists and $\Psi_{\mathfrak{C}} \mathbf{Q}_{\mathfrak{C}}=\operatorname{Id}_{\mathfrak{C}}$. It suffices to show that $\Psi_{\mathfrak{C}} \mathcal{B}\left(\Psi_{\mathfrak{C}}\right)=\Psi_{\mathfrak{C}} \mu_{\mathfrak{C}}=\Psi_{\mathfrak{C}} \Psi_{\mathcal{B}(\mathfrak{C})}$. But this follows directly from the definitions and the fact that $\Psi_{\mathfrak{C}}$ preserves kernels by construction. Conversely suppose that $(\mathfrak{C}, G)$ is an algebra for the triple considered. By Lemma 3.3 it suffices to show that $G$ is a right adjoint of $\mathbf{Q}_{\mathfrak{C}}$. Choose an arbitrary $[A, f, B] \in \mathcal{B}(\mathfrak{C})$, and apply $G$ to the exact sequence (3.2). The complex $G[A, f, B] \stackrel{G\left[1_{A}, 0\right]}{\longrightarrow} G \mathbf{Q}_{\mathfrak{C}}(A) \stackrel{G \mathbf{Q}_{\mathfrak{C}}(f)}{\longrightarrow} G \mathbf{Q}_{\mathfrak{C}}(B)$ induces a morphism $\left[G\left[1_{A}, 0\right], 0\right]: \mathbf{Q}_{\mathfrak{C}}(G[A, f, B]) \rightarrow\left[\mathbf{Q}_{\mathfrak{C}}(A), \mathbf{Q}_{\mathfrak{C}}(f), \mathbf{Q}_{\mathfrak{C}}(B)\right]$ in $\mathcal{B}(\mathfrak{C})$. Since $(\mathfrak{C}, G)$ is an algebra, we have: $G \mathbf{Q}_{\mathfrak{C}}=\operatorname{Id}_{\mathfrak{C}}$. Thus we have a morphism $\left[G\left[1_{A}, 0\right], 0\right]: \mathbf{Q}_{\mathfrak{C}}(G[A, f, B]) \rightarrow[A, f, B]$ in $\mathcal{B}(\mathfrak{C})$. We claim that $\left[G\left[1_{A}, 0\right], 0\right]$ is the component of the counit of an adjoint pair $\left(\mathbf{Q}_{\mathfrak{C}}, G\right)$, with unit the identification $\operatorname{Id}_{\mathfrak{C}}=G \mathbf{Q}_{\mathfrak{C}}$. This is easy and is left to the reader.

Suppose now that $S:(\mathfrak{C}, F) \rightarrow(\mathfrak{D}, G)$ is a morphism of algebras. Then we have $S F=$ $G \mathcal{B}(S)$, and by part (i) we have $F=\Psi_{\mathfrak{C}}, G=\Psi_{\mathfrak{D}}$. Let $0 \rightarrow K \stackrel{g}{\rightarrow} A \stackrel{f}{\rightarrow} B$ be a sequence with $g=k e r(f)$. By construction we have an exact sequence $0 \rightarrow \mathcal{B}(S)[A, f, B] \rightarrow \mathbf{Q}_{\mathfrak{D}} S(A) \stackrel{\mathbf{Q}_{\mathfrak{D}} S(f)}{\longrightarrow}$ $\mathbf{Q}_{\mathfrak{D}} S(B)$ in $\mathfrak{D}$. Applying the kernel preserving functor $\Psi_{\mathfrak{D}}$ to the sequence above we have an exact sequence $0 \rightarrow \Psi_{\mathfrak{D}} \mathcal{B}(S)[A, f, B] \rightarrow S(A) \rightarrow S(B)$ in $\mathfrak{D}$. Since $\Psi_{\mathfrak{D}} \mathcal{B}(S)=S \Psi_{\mathfrak{C}}$, and $\Psi_{\mathfrak{C}}[A, f, B]=\operatorname{Ker}(f)$, we have the exact sequence $0 \rightarrow S(K) \rightarrow S(A) \rightarrow S(B)$ in $\mathfrak{D}$. This proves that $S$ preserves kernels. Conversely if $S: \mathfrak{C} \rightarrow \mathfrak{D}$ is preserves kernels it suffices to prove that $S \Psi_{\mathfrak{C}}=\Psi_{\mathfrak{D}} \mathcal{B}(S)$. This follows trivially from the construction of the functors $\Psi_{\mathfrak{C}}, \Psi_{\mathfrak{D}}, \mathcal{B}(S)$, and the kernel preserving property of $S$. Obviously (i) and (ii) imply that the Eilenberg-Moore comparison functor is an equivalence, thus $\mathcal{J}$ is tripleable.

Remark 3.5. We use throughout additive categories with split idempotents. For the purpose of this paper this is not very essential. We can assume that $\mathfrak{C}$ is an arbitrary category or a preadditive category or an additive category. Indeed if $\mathfrak{C}$ is an arbitrary category, then we can consider the free preadditive category $\mathbb{Z}(\mathfrak{C})$ over $\mathfrak{C}$, then the free additive category $\oplus \mathbb{Z}(\mathfrak{C})$ over $\mathbb{Z}(\mathfrak{C})$, and finally the idempotent completion $\mid \oplus \mathbb{Z}(\mathfrak{C})$ of $\oplus \mathbb{Z}(\mathfrak{C})$. Then we can apply the constructions $\mathcal{A}, \mathcal{B}$ to $\mid \oplus \mathbb{Z}(\mathfrak{C})$.

If an additive category $\mathfrak{D}$ has enough projectives, resp. injectives, we denote by $\mathfrak{D}=$ $\mathfrak{D} / \operatorname{Proj}(\mathfrak{D})$, resp. $\overline{\mathfrak{D}}=\mathfrak{D} / \operatorname{Inj}(\mathfrak{D})$, the stable category of $\mathfrak{D}$ modulo projectives, resp. injectives. We recall that the additive category $\mathfrak{C}$ is called regular (in the sense of von Neumann) if for any morphism $f: A \rightarrow B$ in $\mathfrak{C}$ there exists a morphism $g: B \rightarrow A$ such that: $f \circ g \circ f=f$. 
Proposition 3.6. (1) The category $\mathcal{A}(\mathfrak{C})$, resp. $\mathcal{B}(\mathfrak{C})$, has enough projectives, resp. injectives, and the functor $\mathbf{P}$, resp. $\mathbf{Q}$, induces an equivalence $\mathfrak{C} \approx \operatorname{Proj}(\mathcal{A}(\mathfrak{C}))$, resp. $\mathfrak{C} \approx \operatorname{Inj}(\mathcal{B}(\mathfrak{C})) . \mathfrak{C}$ is regular iff $\mathbf{P}$ is an equivalence iff $\mathbf{Q}$ is an equivalence. If $\mathfrak{C}, \mathfrak{D}$ are two categories (with split idempotents), then: $\mathfrak{C} \approx \mathfrak{D} \Leftrightarrow \mathcal{A}(\mathfrak{C}) \approx \mathcal{A}(\mathfrak{D}) \Leftrightarrow \mathcal{B}(\mathfrak{C}) \approx \mathcal{B}(\mathfrak{D})$.

(2) There are equivalences:

$$
\mathcal{A}\left(\mathfrak{C}^{o p}\right) \approx \mathcal{B}(\mathfrak{C})^{o p}, \quad \mathcal{B}\left(\mathfrak{C}^{o p}\right) \approx \mathcal{A}(\mathfrak{C})^{o p} \quad \text { and } \quad \operatorname{Tr}: \underline{\mathcal{A}(\mathfrak{C})} \underset{\widetilde{\mathcal{B}(\mathfrak{C})}}{ }
$$

Proof. (1) It is easy to see that $\mathbf{P}(A)$ is a projective object and $\left\{0,1_{B}\right\}: \mathbf{P}(B) \rightarrow\{A, f, B\}$ is epic. If $\{A, f, B\} \in \operatorname{Proj}(\mathcal{A}(\mathfrak{C}))$, there exists $\{0, a\}:\{A, f, B\} \rightarrow \mathbf{P}(B)$ with $\{0, a\} \circ\left\{0,1_{B}\right\}=$ $1_{\{A, f, B\}}$. Trivially this is equivalent to the existence of a morphism $b: B \rightarrow A$ with $f=f \circ b \circ f$. Splitting the idempotent $1_{B}-b \circ f$ as $k \circ l$ through an object $X$, it is easy to see that $\{0, k\}:\{A, f, B\} \rightarrow \mathbf{P}(X)$ is an isomorphism. The remaining assertions are left to the reader.

(2) Defining $D\left\{A^{o p}, f^{o p}, B^{o p}\right\}=[B, f, A]$ and $D\left\{a^{o p}, b^{o p}\right\}=[b, a]$, we obtain a well defined functor $D: \mathcal{A}\left(\mathfrak{C}^{o p}\right) \rightarrow \mathcal{B}(\mathfrak{C})^{o p}$ which obviously is an equivalence. Now define $\operatorname{Tr}: \mathcal{A}(\mathfrak{C}) \rightarrow \overline{\mathcal{B}(\mathfrak{C})}$ by $\operatorname{Tr}\{A, f, B\}=\overline{[A, f, B]}$ and $\operatorname{Tr}\{a, b\}=\overline{[a, b]}$. The functor Tr is well defined. For if $\{a, b\}=0$,

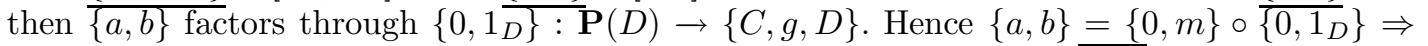
$\{a, m-b\}=0 \Rightarrow \exists \xi: B \rightarrow C$ with $m-b=\xi \circ g$, and $f \circ m=0$. Then $\overline{[a, b]}=0$. Indeed since $(a-f \circ \xi) \circ g=a \circ g-f \circ \xi \circ g=a \circ g-f \circ m-f \circ b=0$, it follows that $[a-f \circ \xi]: \mathbf{Q}(A) \rightarrow[C, g, D]$ is a morphism in $\mathcal{B}(\mathfrak{C})$. Then $[a, b]-\left[1_{A}, 0\right] \circ[a-f \circ \xi]=[f \circ \xi, b]=0$. Hence $[a, b]$ factors through $\mathbf{Q}(A)$, and this means $\overline{[a, b]}=0$. By construction $\operatorname{Tr}$ is full and surjective on objects. The opposite direction of the proof that $\operatorname{Tr}$ is well-defined shows that $\operatorname{Tr}$ is faithful, hence an equivalence.

Lemma 3.7. Let $\mathfrak{D}$ be a category with cokernels (kernels), and $F: \mathfrak{C} \rightarrow \mathfrak{D}$ an be additive functor. Assume $\mathfrak{D}$ is a full subcategory closed under cokernels (kernels) of an abelian category $\mathcal{E}$, such that any object in the image of $F$ is a projective (injective) object of $\mathcal{E}$. Let $F^{!}$: $\mathcal{A}(\mathfrak{C}) \rightarrow \mathfrak{D}\left(F^{*}: \mathcal{B}(\mathfrak{C}) \rightarrow \mathfrak{D}\right)$ be the unique cokernel (kernel) preserving extension of $F$. Then $F^{!}\left(F^{*}\right)$ is an equivalence iff $F$ is fully faithful and any object of $\mathfrak{D}$ is finitely $\operatorname{Im} F$-presented (ImF-copresented).

Proof. If $F^{!}$is an equivalence, then $F$ is fully faithful as the composition of fully faithful functors $\mathbf{P}, F^{\text {! }}$, and by construction any object of $\mathfrak{D}$ appears as a cokernel of a morphism $F(A) \rightarrow F(B)$. Conversely it suffices to show that $F^{!}$is fully faithful. Let $\{a, b\}:\{A, f, B\} \rightarrow$ $\{C, g, D\}$ be a morphism in $\mathcal{A}(\mathfrak{C})$, with $F^{!}\{a, b\}=0$. By construction $F^{!}\{a, b\}$ is the unique morphism in $\mathfrak{D}$ with the property: $c_{F f} \circ F^{!}\{a, b\}=F b \circ c_{F g}$, where $c_{a}=\operatorname{coker}(a)$. Then $F b \circ c_{F g}=0$, hence $F b=k \circ \mathrm{im}(F g)$ in $\mathfrak{E}$. Since $F(B)$ is a projective object of $\mathfrak{E}$, there exists $l: F(B) \rightarrow F(C)$ with $l \circ \operatorname{coim}(F g)=k$. Since $F$ is full, $l=F h$ for some $h: B \rightarrow C$. Then $F h \circ F g=F b$, and since $F$ is faithful, $h \circ g=b$. This means $\{a, b\}=0$ and $F^{!}$is faithful. A similar argument shows that $F^{!}$is full, using that $F$ is full.

Let $(\mathfrak{C}, \mathfrak{X})$ be a left (right) homotopy pair. In the additive category lex $(\mathfrak{X}), \operatorname{resp} . \operatorname{rex}(\mathfrak{X})$, we consider the full subcategory $\operatorname{lex}_{0}(\mathfrak{X}), \operatorname{rex}_{0}(\mathfrak{X})$, consisting of all diagrams $A \stackrel{g}{\rightarrow} B \stackrel{f}{\rightarrow} C$, where $f$, resp. $g$, is split epic, resp. split monic. We denote by lex $(\mathfrak{X})$, rex $(\mathfrak{X})$ the induced stable categories. Let $\underline{\mathcal{E}}^{\mathfrak{X}}$ be the full subcategory of $\mathcal{A}(\mathfrak{C})$ with objects the $\mathfrak{X}$-epics and $\underline{\mathcal{M}}^{\mathfrak{X}}$ be the full subcategory of $\mathcal{B}(\mathfrak{C})$ with objects the $\mathfrak{X}$-monics.

Proposition 3.8. There are equivalences:

$$
{\underline{\mathcal{E}^{\mathfrak{X}}}}^{\mathfrak{A}} \approx \mathcal{A}(\mathfrak{C} / \mathfrak{X}) \approx \underline{\operatorname{lex}}(\mathfrak{X}), \quad \underline{\mathcal{M}}^{\mathfrak{X}} \approx \mathcal{B}(\mathfrak{C} / \mathfrak{X}) \approx \underline{\operatorname{rex}}(\mathfrak{X}) .
$$

Proof. Considering the restriction of the functor $\mathcal{A}(\varpi): \mathcal{A}(\mathfrak{C}) \rightarrow \mathcal{A}(\mathfrak{C} / \mathfrak{X})$ to $\underline{\mathcal{E}}^{\mathfrak{X}}$, where $\varpi$ : $\mathfrak{C} \rightarrow \mathfrak{C} / \mathfrak{X}$ is the projection functor, we obtain a functor $F: \underline{\mathcal{E}}^{\mathfrak{X}} \rightarrow \mathcal{A}(\mathfrak{C} / \mathfrak{X})$. If $\{\underline{A}, \underline{f}, \underline{B}\} \in$ $\mathcal{A}(\mathfrak{C} / \mathfrak{X})$, then consider the object $\left\{A \oplus X_{B},{ }^{t}\left(f, \chi_{B}\right), B\right\} \in \underline{\mathcal{E}}^{\mathfrak{X}}$, where $\chi_{B}: X_{B} \rightarrow B$ is a right $\mathfrak{X}$-approximation of $B$. Then trivially $F\left\{A \oplus X_{B},{ }^{t}\left(f, \chi_{B}\right), B\right\}=\{\underline{A}, \underline{f}, \underline{B}\}$ and $F$ is dense. 
Let $\{a, b\}:\{A, f, B\} \rightarrow\{C, g, D\}$ be a morphism in $\underline{\mathcal{E}}^{\mathfrak{X}}$ with $F\{a, b\}=0$. Then there exists a morphism $\underline{w}: \underline{B} \rightarrow \underline{C}$ with $\underline{w} \circ g=\underline{b}$. This means that $w \circ g-b$ factors through the morphism $\chi_{D}$ as $w \circ g-b=s \circ \chi_{D}$. Since $g$ is $\mathfrak{X}$-epic, we have that $\chi_{D}=t \circ g$ for a morphism $t: X_{D} \rightarrow D$. Then $w \circ g-b=s \circ t \circ g$; hence $b=(w-s \circ t) \circ g$ and this means that $\{a, b\}=0$. We conclude that $F$ is faithful. Finally if $\{\underline{a}, \underline{b}\}:\{\underline{A}, \underline{f}, \underline{B}\} \rightarrow\{\underline{C}, \underline{g}, \underline{D}\}$ is a morphism in $\mathcal{A}(\mathfrak{C} / \mathfrak{X})$, then the morphism $f \circ b-a \circ g$ factors through $\bar{\chi}_{D}$ as $f \circ b-a \circ g=z \circ \chi_{D}$. Let $\chi_{D}=t \circ g$ with $t$ as above. Then $(a+z \circ t) \circ g=a \circ g+z \circ t \circ g=a \circ g+z \circ \chi_{D}=a \circ g+f \circ b-a \circ g=f \circ b$. This means that $\{a+z \circ t, b\}:\{A, f, B\} \rightarrow\{C, g, D\}$ is a morphism in $\underline{\mathcal{E}}^{\mathfrak{X}}$ with the property $F\{a+z \circ t, b\}=\{\underline{a}+\underline{z} \circ \underline{t}, \underline{b}\}=\{\underline{a}, \underline{b}\}$. Hence $F$ is full. We conclude that $F$ is an equivalence of categories. Obviously the equivalence $\mathcal{E}^{\mathfrak{X}} \approx \operatorname{lex}(\mathfrak{X})$ induces an equivalence $\underline{\mathcal{E}}^{\mathfrak{X}} \approx \operatorname{lex}(\mathfrak{X})$. The second part follows by duality.

We recall that a functor $F: \mathfrak{C}^{o p} \rightarrow \mathfrak{A} b$ is called finitely presented if there exists an exact sequence $\mathfrak{C}(-, A) \rightarrow \mathfrak{C}(-, B) \rightarrow F \rightarrow 0$. We denote the category of finitely presented functors by $\bmod -\mathfrak{C}$. If $\mathfrak{C}$ is skeletally small, let $\operatorname{Mod}-\mathfrak{C}$ be the category of contravariant additive functors from $\mathfrak{C}$ to the category $\mathfrak{A} b$ of abelian groups and let $\operatorname{Lex}\left(\mathfrak{C}^{o p}, \mathfrak{A} b\right), \operatorname{resp} \cdot \operatorname{Rex}\left(\mathfrak{C}^{o p}, \mathfrak{A} b\right)$, be the full subcategories of kernel, resp. cokernel, preserving functors. The next result follows from Corollary 3.2 and Lemma 3.7.

Corollary 3.9. (i) The extension $\mathrm{Y}^{!}: \mathcal{A}(\mathfrak{C}) \rightarrow$ Mod-c $\mathfrak{C}$ of the Yoneda embedding $\mathrm{Y}: \mathfrak{C} \rightarrow$ Mod-C induces an equivalence $\mathcal{A}(\mathfrak{C}) \approx \bmod -\mathfrak{C}$ and further $\mathcal{B}(\mathfrak{C})^{\text {op }} \approx \mathfrak{C}-\bmod$. In particular $\mathcal{A}(\mathfrak{C}), \mathcal{B}(\mathfrak{C})$ are exact categories in the sense of Quillen.

(ii) There are equivalences:

$$
\begin{aligned}
\mathfrak{C}-\operatorname{Mod} & \approx \operatorname{Rex}[\mathcal{A}(\mathfrak{C}), \mathfrak{A} b] \approx \operatorname{Lex}[\mathcal{B}(\mathfrak{C}), \mathfrak{A} b] \\
\operatorname{Mod}-\mathfrak{C} & \approx \operatorname{Lex}\left[\mathcal{A}(\mathfrak{C})^{o p}, \mathfrak{A} b\right] \approx \operatorname{Rex}\left[\mathcal{B}(\mathfrak{C})^{o p}, \mathfrak{A} b\right] .
\end{aligned}
$$

If $\Lambda$ is an associative ring, we denote by $\mathcal{P}_{\Lambda}$ the category of finitely generated projective right modules. By Corollary $3.9, \mathcal{A}\left(\mathcal{P}_{\Lambda}\right) \approx \bmod -\Lambda$ is the category of finitely presented right $\Lambda$-modules and, using the well known duality $\mathcal{P}_{\Lambda^{o p}} \approx\left(\mathcal{P}_{\Lambda}\right)^{o p}$, it follows that $\mathcal{B}\left(\mathcal{P}_{\Lambda}\right) \approx$ $\mathcal{B}\left(\left(\mathcal{P}_{\Lambda^{o p}}\right)^{o p}\right) \approx \mathcal{A}\left(\mathcal{P}_{\Lambda^{o p}}\right)^{o p} \approx(\Lambda-\bmod )^{o p}$. In this case the equivalence $\mathrm{Tr}$ of Proposition 3.6 is the Auslander-Bridger transpose duality functor between the stable categories $\underline{\bmod }-\Lambda$, $\Lambda-\underline{\bmod }$ modulo projectives [5].

For an abelian category $\mathfrak{C}$ we denote by $\underline{\operatorname{Sex}}(\mathfrak{C})$ the stable category of the category Sex of short exact sequences, modulo the split short exact sequences. The following is a consequence of Lemma 3.7 and Proposition 3.8.

Corollary 3.10. If $\mathfrak{C}$ is an abelian category with enough projectives, resp. injectives, then there are equivalences:

$$
\begin{gathered}
\mathfrak{C} \approx \mathcal{A}(\operatorname{Proj}(\mathfrak{C})) \text { and } \mathcal{A}(\mathfrak{C} / \operatorname{Proj}(\mathfrak{C})) \approx \underline{\operatorname{Sex}}(\mathfrak{C}) \\
\text { resp. } \quad \mathfrak{C} \approx \mathcal{B}(\operatorname{Inj}(\mathfrak{C})) \text { and } \mathcal{B}(\mathfrak{C} / \operatorname{Inj}(\mathfrak{C})) \approx \underline{\operatorname{Sex}}(\mathfrak{C})
\end{gathered}
$$

\section{Coherence}

An additive category $\mathfrak{C}$ is called right (left) coherent iff $\mathfrak{C}$ has weak kernels (weak cokernels), and is called coherent if $\mathfrak{C}$ is left and right coherent. We recall that a weak kernel of a morphism $f: A \rightarrow B$ in $\mathfrak{C}$ is a morphism $g: K \rightarrow A$ with $g \circ f=0$, such that for any morphism $h: X \rightarrow A$ with $h \circ f=0$, there exists $t: X \rightarrow K$ with $t \circ g=h$. Weak cokernels are defined as weak kernels in $\mathfrak{C}^{o p}$.

Definition 4.1. A pair $(\mathfrak{C}, \mathfrak{D})$ of additive categories is called a Morita pair if there exists an equivalence $\mathcal{A}(\mathfrak{C}) \approx \mathcal{B}(\mathfrak{D})$. If $(\mathfrak{C}, \mathfrak{D})$ is a Morita pair then $\mathfrak{C}$, resp. $\mathfrak{D}$, is called a right Morita, 
resp. left Morita category. An additive category $\mathfrak{C}$ is called a dualizing category if the pair $(\mathfrak{C}, \mathfrak{C})$ is Morita, i.e. if $\mathfrak{C}$ has equivalent Freyd categories.

Observe that by Proposition 3.6 the categories ocuuring in a Morita pair are uniquely determined. We note that the pair $(\mathfrak{C}, \mathfrak{D})$ is Morita iff the pair $\left(\mathfrak{D}^{o p}, \mathfrak{C}^{o p}\right)$ is Morita. Indeed this follows from the dualities $\mathcal{B}\left(\mathfrak{C}^{o p}\right) \approx \mathcal{A}(\mathfrak{C})^{o p}, \mathcal{A}\left(\mathfrak{D}^{o p}\right) \approx \mathcal{B}(\mathfrak{D})^{o p}$.

Example 4.2. Let $\Lambda, \Gamma$ be associative rings and $\mathcal{P}_{\Lambda}, \mathcal{P}_{\Gamma}$ be the categories of finitely generated projective right modules over $\Lambda, \Gamma$. Since $\mathcal{A}\left(\mathcal{P}_{\Lambda}\right) \approx \bmod -\Lambda$, and $\mathcal{B}\left(\mathcal{P}_{\Gamma}\right) \approx \mathcal{B}\left(\left({ }_{\Gamma} \mathcal{P}\right)^{o p}\right) \approx$ $\mathcal{A}\left({ }_{\Gamma} \mathcal{P}\right)^{o p} \approx(\Gamma-\bmod )^{o p}$, it follows that the pair $\left(\mathcal{P}_{\Lambda}, \mathcal{P}_{\Gamma}\right)$ is Morita iff there exists a duality $\bmod -\Lambda \rightarrow \Gamma-$ mod. We recall that a ring $\Lambda$ is called a right Morita ring if $\Lambda$ is right Noetherian and there exists a finitely generated injective cogenerator in Mod $-\Lambda$. It follows directly that $\Lambda$ is right Morita iff $\Lambda$ is right Noetherian and $\mathcal{P}_{\Lambda}$ is a right Morita category. The category $\mathcal{P}_{\Gamma}$ is dualizing iff there exists a duality $\Gamma-\bmod \rightarrow \bmod -\Gamma$. Hence if $\Lambda$ is a Quasi-Frobenius ring, QF-ring for short, or an Artin algebra, then the category $\mathcal{P}_{\Lambda}$ is dualizing.

Example 4.3. If $\mathcal{H}$ is an abelian category with enough projective and injective objects, then the pair $(\operatorname{Proj}(\mathcal{H}), \operatorname{Inj}(\mathcal{H}))$ is Morita. This follows from the equivalences $\mathcal{A}(\operatorname{Proj}(\mathcal{H})) \approx$ $\mathcal{H} \approx \mathcal{B}(\operatorname{Inj}(\mathcal{H}))$. We recall that $\mathcal{H}$ is called Frobenius if $\operatorname{Proj}(\mathcal{H})=\operatorname{Inj}(\mathcal{H})$. It follows that the category of projective (= injective) objects in a Frobenius abelian category $\mathcal{H}$ is dualizing. So for any QF-ring $\Lambda$, the category $\operatorname{Proj}(\operatorname{Mod}-\Lambda)$ of all projective right $\Lambda$-modules is dualizing.

Example 4.4. If $\mathfrak{C}$ is a dualizing $R$-variety in the sense of $[6]$, then it is easy to see that $\mathfrak{C}$ is a dualizing category. Note that important examples of dualizing $R$-varieties are the categories of finitely generated projective right modules and the category of finitely generated right modules over an Artin algebra.

Proposition 4.5. (1) The category $\mathcal{A}(\mathfrak{C})$ is abelian iff $\mathfrak{C}$ is right coherent. The category $\mathcal{B}(\mathfrak{C})$ is abelian iff $\mathfrak{C}$ is left coherent.

(2) An additive category $\mathfrak{C}$ is right, resp. left, Morita iff $\mathfrak{C}$ is right, resp. left, coherent and $\mathcal{A}(\mathfrak{C})$, resp. $\mathcal{B}(\mathfrak{C})$, has enough injectives, resp. projectives.

(3) Suppose that $\mathcal{A}(\mathfrak{C})$ is abelian. Then $\operatorname{gl} \cdot \operatorname{dim} \mathcal{A}(\mathfrak{C})=0$ iff $\mathfrak{C}$ is semisimple abelian, in which case $\mathfrak{C} \approx \mathcal{A}(\mathfrak{C})$. The same is true for $\mathcal{B}(\mathfrak{C})$.

(4) If $\mathfrak{C}$ has kernels, resp. cokernels, then: $\operatorname{gl} \operatorname{dim} \mathcal{A}(\mathfrak{C})=1$, resp. $\operatorname{gl} \cdot \operatorname{dim} \mathcal{B}(\mathfrak{C})=1$, iff $\mathfrak{C}$ is not semisimple abelian and for any morphism $f: A \rightarrow B$ in $\mathfrak{C}, k e r(f)$, resp. coker $(f)$, is split monic, resp. epic.

(5) If $\mathfrak{C}$ is abelian non-semisimple, then: $\operatorname{gl} . \operatorname{dim} \mathcal{A}(\mathfrak{C})=\operatorname{gl} \cdot \operatorname{dim} \mathcal{B}(\mathfrak{C})=2$.

(6) The category $\mathfrak{C}$ has kernels, resp. cokernels, iff the category $\mathcal{A}(\mathfrak{C})$, resp. $\mathcal{B}(\mathfrak{C})$ is abelian and $\operatorname{gl} \cdot \operatorname{dim} \mathcal{A}(\mathfrak{C}) \leqslant 2$, resp. gl.dim $\mathcal{B}(\mathfrak{C}) \leqslant 2$.

Proof. (1), (2) For a proof of (1) we refer to [20]. If $\mathfrak{C}$ is right coherent and $\mathcal{A}(\mathfrak{C})$ has injectives then by $(1), \mathcal{A}(\mathfrak{C})$ is abelian and if $\mathfrak{D}$ is the full subcategory of injectives, then by Corollary 3.10 we have $\mathcal{A}(\mathfrak{C}) \approx \mathcal{B}(\mathfrak{D})$. Conversely if $\mathfrak{C}$ is right Morita and $\mathcal{A}(\mathfrak{C}) \approx \mathcal{B}(\mathfrak{D})$, then since $\mathcal{B}(\mathfrak{D})$ has kernels and injectives the same is true for $\mathcal{A}(\mathfrak{C})$. Then $\mathfrak{C}$ is right coherent, since trivially $\mathcal{A}(\mathfrak{C})$ has kernels if and only if $\mathfrak{C}$ has weak kernels. The parenthetical case is dual.

(3) If gl.dim $\mathcal{A}(\mathfrak{C})=0$, then any object in $\mathcal{A}(\mathfrak{C})$ is projective, so by Proposition 3.6, $\mathbf{P}$ : $\mathfrak{C} \rightarrow \mathcal{A}(\mathfrak{C})$ is an equivalence. If $\mathfrak{C}$ is semisimple abelian, then $\forall\{A, f, B\} \in \mathcal{A}(\mathfrak{C})$, choose $a: B \rightarrow \operatorname{Im}(f), b: \operatorname{Im}(f) \rightarrow A$, with $i m(f) \circ a=1_{\operatorname{Im}(f)}, b \circ \operatorname{coim}(f)=1_{\operatorname{Im}(f)}$, and consider the morphism $g=a \circ b: B \rightarrow A$. Then $f=f \circ g \circ f$, and $\left\{0,1_{B}-g \circ f\right\}:\{A, f, B\} \rightarrow \mathbf{P}(B)$ is a morphism in $\mathcal{A}(\mathfrak{C})$ with $\left\{0,1_{B}-g \circ f\right\} \circ\left\{0,1_{B}\right\}=1_{\{A, f, B\}}$. Hence $\{A, f, B\}$ is projective, and $\operatorname{gl} \operatorname{dim} \mathcal{A}(\mathfrak{C})=0$. The last assertion follows from Proposition 3.6(1). 
(4) If $\mathfrak{C}$ has kernels then $\mathcal{A}(\mathfrak{C})$ is abelian by (1). Suppose that gl.dim $\mathcal{A}(\mathfrak{C})=1$, and let $0 \neq\{A, f, B\} \in \mathcal{A}(\mathfrak{C})$. If $g: K \rightarrow A$ is the kernel of $f$, since $\mathbf{P}$ preserves kernels, the following is a projective resolution of $\{A, f, B\}$ :

$$
0 \longrightarrow \mathbf{P}(K) \stackrel{\mathbf{P}(g)}{\longrightarrow} \mathbf{P}(A) \stackrel{\mathbf{P}(f)}{\longrightarrow} \mathbf{P}(B) \stackrel{\left\{0,1_{B}\right\}}{\longrightarrow}\{A, f, B\} \longrightarrow 0
$$

Since gl. $\operatorname{dim} \mathcal{A}(\mathfrak{C})=1$, the first syzygy of $\{A, f, B\}$ which as easily seen is $\{K, g, A\}$, is projective. Hence $\exists h: A \rightarrow K$ with $g=g \circ h \circ g$. Since $g$ is monic, $1_{K}=g \circ h$, and $g$ is split monic. Conversely if $\forall\{A, f, B\} \in \mathcal{A}(\mathfrak{C}), g=k e r(f)$ is split monic, then $\exists h: A \rightarrow K$ with $g=g \circ h \circ g$. This implies that $\{K, g, A\}$ is projective. Hence p.d $\{A, f, B\} \leqslant 1$, and since $\mathcal{A}(\mathfrak{C})$ is not semisimiple, gl.dim $\mathcal{A}(\mathfrak{C})=1$.

(5) Follows easily from (4). (6) If $\mathfrak{C}$ has kernels then by (1), $\mathcal{A}(\mathfrak{C})$ is abelian and the proof of (4) shows that gl.dim $\mathcal{A}(\mathfrak{C}) \leqslant 2$. Conversely if $\mathcal{A}(\mathfrak{C})$ is abelian and $\operatorname{gl} \operatorname{dim} \mathcal{A}(\mathfrak{C}) \leqslant 2$, then obviously the morphism $g$ in the projective resolution (1) of part (4) above, is the kernel of $f$ since $\mathbf{P}$ is fully faithful. Dually for $\mathcal{B}(\mathfrak{C})$.

¿From the above Proposition and Lemma 3.3, we have directly the following.

Corollary 4.6. An additive category $\mathfrak{C}$ has kernels and cokernels iff $\mathfrak{C}$ is the full reflective (coreflective) subcategory of all projective (injective) objects of an abelian category $\mathfrak{D}$ with enough projectives (injectives) and gl.dimD $\leqslant 2$.

Suppose now that $f: A \rightarrow B$ is a morphism in the additive category $\mathfrak{C}$. A weak kernel sequence over $f$ is a complex in $\mathfrak{C}$ :

$$
K_{f}^{\bullet}: \quad \cdots \rightarrow K_{f}^{n} \stackrel{k_{f}^{n}}{\longrightarrow} K_{f}^{n-1} \rightarrow \cdots \rightarrow K_{f}^{0} \stackrel{k_{f}^{0}}{\longrightarrow} A \stackrel{f}{\rightarrow} B
$$

in which each morphism is a weak kernel of the next. Dually a weak cokernel sequence under $f$ is a complex in $\mathfrak{C}$ :

$$
C_{\bullet}^{f}: \quad A \stackrel{f}{\rightarrow} B \stackrel{c_{0}^{f}}{\longrightarrow} C_{0}^{f} \rightarrow \cdots \rightarrow C_{n-1}^{f} \stackrel{c_{n}^{f}}{\longrightarrow} C_{n}^{f} \rightarrow \cdots
$$

in which each morphism is a weak cokernel of the preceding.

Corollary 4.7. (1) $\mathbf{P}\left[K_{f}^{\bullet}\right] \rightarrow\{A, f, B\}$ is a projective resolution of $\{A, f, B\}$ in $\mathcal{A}(\mathfrak{C})$, and $[A, f, B] \rightarrow \mathbf{Q}\left[C_{\bullet}^{f}\right]$ is an injective resolution of $[A, f, B]$ in $\mathcal{B}(\mathfrak{C})$.

(2) If $\mathfrak{C}$ is right (left) coherent and if $F: \mathfrak{C} \rightarrow \mathfrak{D}$ is an additive functor to an abelian category $\mathfrak{D}$, then $F^{!}: \mathcal{A}(\mathfrak{C}) \rightarrow \mathfrak{D}\left(F^{*}: \mathcal{B}(\mathfrak{C}) \rightarrow \mathfrak{D}\right)$ is exact iff $F$ sends weak kernel (weak cokernel) sequences in $\mathfrak{C}$ to exact sequences in $\mathfrak{D}$.

(3) If $\mathfrak{C}$ is right (left) coherent with cokernels (kernels), then the left (right) adjoint $\Phi$ : $\mathcal{A}(\mathfrak{C}) \rightarrow \mathfrak{C}(\Psi: \mathcal{B}(\mathfrak{C}) \rightarrow \mathfrak{C})$ of $\mathbf{P}(\mathbf{Q})$ is exact.

Proof. (1) Since any projective in $\mathcal{A}(\mathfrak{C})$ is of the form $\mathbf{P}(X)$, it is enough to prove that $\mathcal{A}(\mathfrak{C})\left[\mathbf{P}(X), \mathbf{P}\left[K_{f}^{\bullet}\right]\right]$ is exact in $\mathfrak{A} b, \forall X \in \mathfrak{C}$. Since $\mathbf{P}$ is fully faithful, $\mathcal{A}(\mathfrak{C})\left[\mathbf{P}(X), \mathbf{P}\left[K_{f}^{\bullet}\right]\right] \cong$ $\mathfrak{C}\left[X, K_{f}^{\bullet}\right]$ which is exact by the definition of weak kernels.

(2), (3) If $\mathfrak{C}$ right coherent, $\mathcal{A}(\mathfrak{C})$ is abelian. Then $F^{!}$is exact iff $\mathcal{L}_{i} F^{!}=0, \forall i \geqslant 1$. But $\mathcal{L}_{i} F^{!}(\{A, f, B\})=H_{i}\left(F^{!} \mathbf{P}\left[K_{f}^{\bullet}\right]\right)=H_{i}\left(F\left[K_{f}^{\bullet}\right]\right)$. Hence $F^{!}$is exact iff $F\left[K_{f}^{\bullet}\right]$ is acyclic, from which the assertion follows. Part (3) is trivial.

Using the complexes $K_{f}^{\bullet}, C_{\bullet}^{g}$, we see easily that the syzygy objects and the cosyzygy objects of $\{A, f, B\} \in \mathcal{A}(\mathfrak{C}),[C, g, D] \in \mathcal{B}(\mathfrak{C})$ are respectively:

$$
\begin{gathered}
\Omega^{n+1}\{A, f, B\}=\left\{K_{n}^{f}, k_{n}^{f}, K_{n-1}^{f}\right\}, \forall n \geqslant 1, \quad \Omega^{1}\{A, f, B\}=\left\{K_{0}^{f}, k_{0}^{f}, A\right\} \\
\Sigma^{n+1}[C, g, D]=\left[C_{g}^{n-1}, c_{g}^{n}, C_{g}^{n}\right], \forall n \geqslant 1, \quad \Sigma^{1}[C, g, D]=\left[D, c_{g}^{0}, C_{g}^{0}\right] .
\end{gathered}
$$


If $\mathfrak{C}$ is left coherent then $\mathcal{B}(\mathfrak{C})$ is abelian, hence by the universal property of $\mathcal{A}(\mathfrak{C})$, there exists a unique cokernel preserving functor $\mathbf{Q}^{!}: \mathcal{A}(\mathfrak{C}) \rightarrow \mathcal{B}(\mathfrak{C})$ with $\mathbf{Q}^{!} \mathbf{P}=\mathbf{Q}$. If $\mathfrak{C}$ is right coherent then $\mathcal{A}(\mathfrak{C})$ is abelian, hence there exists a unique kernel preserving functor $\mathbf{P}^{*}: \mathcal{B}(\mathfrak{C}) \rightarrow \mathcal{A}(\mathfrak{C})$ such that $\mathbf{P}^{*} \mathbf{Q}=\mathbf{P}$.

In case $\mathfrak{C}=\mathcal{P}_{\Lambda}$, we have: $\Lambda$ is right coherent iff $\mathcal{P}_{\Lambda}$ has weak kernels, and $\Lambda$ is left coherent iff $\mathcal{P}_{\Lambda^{o p}}$ has weak kernels iff $\mathcal{P}_{\Lambda}$ has weak cokernels. Then we have identifications $\mathbf{Q}^{!}=\operatorname{Hom}_{\Lambda}(-, \Lambda): \bmod -\Lambda \rightarrow \Lambda-\bmod$ and $\mathbf{P}^{*}=\operatorname{Hom}_{\Lambda}(-, \Lambda): \Lambda-\bmod \rightarrow \bmod -\Lambda$, and the sequences of the next Corollary are generalizations of well known exact sequences in case $\mathfrak{C}=\mathcal{P}_{\Lambda}($ see $[5])$.

Corollary 4.8. We have an adjoint pair $\left(\mathbf{Q}^{!}, \mathbf{P}^{*}\right)$, exact sequences:

$$
\begin{gathered}
0 \longrightarrow \mathcal{R}^{1} \mathbf{P}^{*} \operatorname{Tr} \longrightarrow \operatorname{Id}_{\mathcal{A}(\mathfrak{C})} \longrightarrow \mathbf{P}^{*} \mathbf{Q}^{!} \longrightarrow \mathcal{R}^{2} \mathbf{P}^{*} \operatorname{Tr} \longrightarrow 0, \\
0 \longrightarrow \mathcal{L}^{2} \mathbf{Q}^{!} \operatorname{Tr} \longrightarrow \mathbf{Q}^{!} \mathbf{P}^{*} \longrightarrow \operatorname{Id}_{\mathcal{B}(\mathfrak{C})} \longrightarrow \mathcal{L}^{1} \mathbf{Q}^{!} \operatorname{Tr} \longrightarrow 0,
\end{gathered}
$$

and natural isomorphisms: $\mathcal{E} x t_{\mathcal{A}(\mathfrak{C})}^{n}[?, \mathbf{P}-] \cong \mathcal{L}_{n} \mathbf{Q}^{!}(?), \quad \mathcal{E} x t_{\mathcal{B}(\mathfrak{C})}^{n}[\mathbf{Q}-, ?] \cong \mathcal{R}^{n} \mathbf{P}^{*}(?), \forall n \geqslant 1$, where we view the objects $\mathcal{R}^{n} \mathbf{P}^{*}(?), \mathcal{L}_{n} \mathbf{Q}^{!}(?)$ as objects of $\bmod -\mathfrak{C}, \mathfrak{C}-\bmod$ respectively, under the equivalences of Corollary 3.9.

Proof. Consider arbitrary objects $\{A, f, B\} \in \mathcal{A}(\mathfrak{C}),[C, g, D] \in \mathcal{B}(\mathfrak{C})$. By the construction of $\mathbf{Q}^{!}, \mathbf{P}^{*}$, we have exact sequences:

$$
\begin{gathered}
0 \rightarrow \mathbf{P}^{*}[C, g, D] \rightarrow \mathbf{P}(C) \stackrel{\mathbf{P}(g)}{\longrightarrow} \mathbf{P}(D) \stackrel{\left\{0,1_{D}\right\}}{\longrightarrow}\{C, g, D\} \rightarrow 0 \\
0 \longrightarrow[A, f, B] \stackrel{\left[1_{A}, 0\right]}{\longrightarrow} \mathbf{Q}(A) \stackrel{\mathbf{Q}(f)}{\longrightarrow} \mathbf{Q}(B) \longrightarrow \mathbf{Q}^{!}\{A, f, B\} \longrightarrow 0
\end{gathered}
$$

Applying to (4.5) the functor $\mathbf{P}^{*}$ we have the complex:

$$
0 \longrightarrow \mathbf{P}^{*}[A, f, B] \longrightarrow \mathbf{P}(A) \stackrel{\mathbf{P}(f)}{\longrightarrow} \mathbf{P}(B) \stackrel{a}{\rightarrow} \mathbf{P}^{*} \mathbf{Q}^{!}\{A, f, B\} \longrightarrow 0
$$

so there exists a unique morphism $\delta:\{A, f, B\} \rightarrow \mathbf{P}^{*} \mathbf{Q}^{!}\{A, f, B\}$, with $\left\{0,1_{B}\right\} \circ \delta=a$. Since $\mathbf{P}^{*}$ is left exact, $\operatorname{Ker}(a)=\mathbf{P}^{*} \Sigma[A, f, B]$, and the cokernel of the induced morphism $\mathbf{P}(A) \rightarrow$ $\mathbf{P}^{*} \Sigma[A, f, B]$ is $\mathcal{R}^{1} \mathbf{P}^{*}[A, f, B]=\mathcal{R}^{1} \mathbf{P}^{*} \operatorname{Tr}\{A, f, B\}$. Moreover Coker $(\delta)=\operatorname{Coker}\left(\left\{0,1_{B}\right\} \circ \delta\right)$ $=$ Coker $(a)=\mathcal{R}^{2} \mathbf{P}^{*}[A, f, B]=\mathcal{R}^{2} \mathbf{P}^{*} \operatorname{Tr}\{A, f, B\}$. Similarly $\forall[C, g, D] \in \mathcal{B}(\mathfrak{C})$, there exists a morphism $\varepsilon: \mathbf{Q}^{!} \mathbf{P}^{*}[C, g, D] \rightarrow[C, g, D]$, with $\operatorname{Ker}(\varepsilon)=\mathcal{L}_{2} \mathbf{Q}^{!}\{C, g, D\}=\mathcal{L}_{2} \mathbf{Q}^{!} \operatorname{Tr}[C, g, D]$, and $\operatorname{Coker}(\varepsilon)=\mathcal{L}_{1} \mathbf{Q}^{!}\{C, g, D\}=\mathcal{L}_{1} \mathbf{Q}^{!} \operatorname{Tr}[C, g, D]$. It is easy to see that $\varepsilon, \delta$ are natural and $\varepsilon$ is the counit and $\delta$ is the unit of an adjoint pair $\left(\mathbf{Q}^{!}, \mathbf{P}^{*}\right)$. Finally $\mathcal{E} x t_{\mathcal{A}(\mathfrak{C})}^{n}[\{A, f, B\}, \mathbf{P}-] \cong$ $H^{n}\left[\mathbf{P}\left(K_{f}^{\bullet}\right), \mathbf{P}(-)\right]$. Since $\mathbf{P}$ is fully faithful, $H^{n}\left[\mathbf{P}\left(K_{f}^{\bullet}\right), \mathbf{P}(-)\right]$ is isomorphic to the complex:

$$
0 \rightarrow \mathfrak{C}(B,-) \rightarrow \mathfrak{C}(A,-) \rightarrow \mathfrak{C}\left(K_{0}^{f}\right) \rightarrow \cdots
$$

in $\mathfrak{C}-\bmod$ and $\mathcal{L}_{n} \mathbf{Q} !\{A, f, B\}$ is the homology of the complex:

$$
\cdots \rightarrow \mathbf{Q}\left(K_{0}^{f}\right) \rightarrow \mathbf{Q}(A) \rightarrow \mathbf{Q}(B) \rightarrow 0
$$

which is isomorphic to the complex above, using the duality $\mathcal{B}(\mathfrak{C})^{o p} \approx \mathfrak{C}-\bmod$.

If $\mathfrak{C}$ is a coherent category, consider the full subcategories

$$
\begin{gathered}
\mathcal{R} \operatorname{efl}(\mathcal{A}(\mathfrak{C}))=\left\{\{A, f, B\} \in \mathcal{A}(\mathfrak{C}) \mid \delta_{\{A, f, B\}}:\{A, f, B\} \stackrel{\cong}{\longrightarrow} \mathbf{P}^{*} \mathbf{Q}^{!}\{A, f, B\}\right\} \\
\operatorname{Refl}(\mathcal{B}(\mathfrak{C}))=\left\{[C, g, D] \in \mathcal{B}(\mathfrak{C}) \mid \varepsilon_{[C, g, D]}: \mathbf{Q}^{!} \mathbf{P}^{*}[C, g, D] \stackrel{\cong}{\longrightarrow}[C, g, D]\right\}
\end{gathered}
$$

consisting of the reflexive objects. Then we have inclusions $\operatorname{Proj}(\mathcal{A}(\mathfrak{C})) \subseteq \mathcal{R e f l}(\mathcal{A}(\mathfrak{C}))$ and $\operatorname{Inj}(\mathcal{B}(\mathfrak{C})) \subseteq \mathcal{R} \operatorname{efl}(\mathcal{B}(\mathfrak{C}))$, and the adjoint pair $\left(\mathbf{Q}^{!}, \mathbf{P}^{*}\right)$ induces inverse equivalences

$$
\mathbf{Q}^{!}: \mathcal{R} \operatorname{efl}(\mathcal{A}(\mathfrak{C})) \stackrel{\approx}{\longrightarrow} \operatorname{Refl}(\mathcal{B}(\mathfrak{C})), \quad \mathbf{P}^{*}: \operatorname{Refl}(\mathcal{B}(\mathfrak{C})) \stackrel{\approx}{\longrightarrow} \operatorname{Refl}(\mathcal{A}(\mathfrak{C}))
$$


Corollary 4.9. Suppose that $\mathfrak{C}$ is a coherent category.

(i) $\mathbf{Q}^{!}$is exact $\Leftrightarrow \mathbf{P}^{*}$ is full and faithful $\Leftrightarrow$ any projective in $\mathcal{A}(\mathfrak{C})$ is injective $\Leftrightarrow \mathcal{R}$ efl $(\mathcal{B}(\mathfrak{C}))$ $=\mathcal{B}(\mathfrak{C}) \Leftrightarrow$ any morphism in $\mathfrak{C}$ is a weak cokernel.

(ii) $\mathbf{P}^{*}$ is exact $\Leftrightarrow \mathbf{Q}^{!}$is full and faithful $\Leftrightarrow$ any injective in $\mathcal{B}(\mathfrak{C})$ is projective $\Leftrightarrow \operatorname{Refl}(\mathcal{A}(\mathfrak{C}))$ $=\mathcal{A}(\mathfrak{C}) \Leftrightarrow$ any morphism in $\mathfrak{C}$ is a weak kernel.

(iii) $\mathbf{P}^{*}, \mathbf{Q}^{!}$are exact $\Leftrightarrow \mathbf{P}^{*}, \mathbf{Q}^{!}$are equivalences $\Leftrightarrow$ the abelian categories $\mathcal{A}(\mathfrak{C}), \mathcal{B}(\mathfrak{C})$ are Frobenius $\Leftrightarrow \mathcal{A}(\mathfrak{C})=\mathcal{R} \operatorname{efl}(\mathcal{A}(\mathfrak{C}))$ and $\operatorname{Refl}(\mathcal{B}(\mathfrak{C}))=\mathcal{B}(\mathfrak{C}) \Leftrightarrow$ any morphism in $\mathfrak{C}$ is a weak kernel and a weak cokernel.

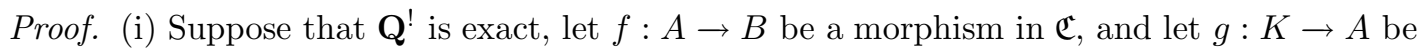
a weak kernel of $f$. Then $\mathbf{P}(K) \stackrel{\mathbf{P}(g)}{\longrightarrow} \mathbf{P}(A) \stackrel{\mathbf{P}(f)}{\longrightarrow} \mathbf{P}(B)$ is exact; then since $\mathbf{Q}^{!}$is exact and $\mathbf{Q} ! \mathbf{P}=\mathbf{Q}$, the sequence $\mathbf{Q}(K) \stackrel{\mathbf{Q}(g)}{\longrightarrow} \mathbf{Q}(A) \stackrel{\mathbf{Q}(f)}{\longrightarrow} \mathbf{Q}(B)$ is exact. This means that $f$ is a weak cokernel of $g$. Conversely if any morphism in $\mathfrak{C}$ is a weak cokernel, let $\{A, f, B\} \in \mathcal{A}(\mathfrak{C})$ and consider the projective resolution $K_{f}^{\bullet} \rightarrow\{A, f, B\} \rightarrow 0$ arising from a weak kernel sequence $K_{f}^{\bullet}$ over $f$. Applying to this resolution the functor $\mathbf{Q}^{\text {! }}$, we obtain the complex $(*): \cdots \rightarrow$ $\mathbf{Q}\left(K_{f}^{0}\right) \stackrel{\mathbf{Q}\left(k_{f}^{0}\right)}{\longrightarrow} \mathbf{Q}(A) \stackrel{\mathbf{Q}(f)}{\longrightarrow} \mathbf{Q}(B)$. Since any morphism in $\mathfrak{C}$ is a weak cokernel, let $h: X \rightarrow A$ be a morphism in $\mathfrak{C}$ with $f$ as a weak cokernel. Then $h=a \circ k_{f}^{0}$, since $k_{f}^{0}$ is a weak kernel of $f$. Then if $g: A \rightarrow Y$ is a morphism with $k_{f}^{0} \circ g=0$, we have $h \circ g=a \circ k_{f}^{0} \circ g=0$. Then $g$ factors through $f$ since $f$ is a weak cokernel of $h$, and this means that $f$ is a weak cokernel of its weak kernel $k_{f}^{0}$. Hence in the complex $\cdots \rightarrow K_{f}^{0} \stackrel{k_{f}^{0}}{\longrightarrow} A \stackrel{f}{\longrightarrow} B$ any morphism is a weak cokernel of its preceding. This means that the complex $(*)$ is exact and consequently the functor $\mathbf{Q}^{!}$is exact.

The other parts of (i) are direct consequences of the exact sequences and isomorphisms of Corollary 4.8, part (ii) is dual and (iii) follows from (i), (ii).

Assume now that $\mathfrak{C}$ is abelian. Then by Corollary 4.7, the left adjoint $\Phi: \mathcal{A}(\mathfrak{C}) \rightarrow \mathfrak{C}$ is exact. Hence $\operatorname{Ker}\left(\Phi_{\mathfrak{C}}\right):=\mathcal{A}_{e}(\mathfrak{C})=\{\{A, f, B\} \in \mathcal{A}(\mathfrak{C}): f$ is epic $\}$ is a localizing subcategory of $\mathcal{A}(\mathfrak{C})$ and $\Phi$ induces an equivalence $\mathcal{A}(\mathfrak{C}) / \mathcal{A}_{e}(\mathfrak{C}) \stackrel{\widetilde{\approx}}{\rightarrow} \mathfrak{C}$. Similarly the right adjoint $\Psi: \mathcal{B}(\mathfrak{C}) \rightarrow \mathfrak{C}$ is exact and $\operatorname{Ker}\left(\Psi_{\mathfrak{C}}\right):=\mathcal{B}^{m}(\mathfrak{C})=\{[A, f, B] \in \mathcal{B}(\mathfrak{C}): f$ is monic $\}$ is a colocalizing subcategory of $\mathcal{B}(\mathfrak{C})$ and $\Psi$ induces an equivalence $\mathcal{B}(\mathfrak{C}) / \mathcal{B}^{m}(\mathfrak{C}) \stackrel{\approx}{\rightarrow} \mathfrak{C}$. The next result gives simple proofs of the duality theorem of [3, p.154], and of a theorem of [20].

Corollary 4.10. For any abelian category $\mathfrak{C}$ there are equivalences

$$
\mathcal{A}_{e}(\mathfrak{C}) \stackrel{\approx}{\operatorname{Sex}}(\mathfrak{C}) \stackrel{\approx}{\approx} \mathcal{B}^{m}(\mathfrak{C}) .
$$

In particular $\underline{\operatorname{Sex}}(\mathfrak{C})$ is abelian. If $\mathfrak{C}$ has enough projectives and injectives, then:

$$
\mathcal{A}(\mathfrak{C} / \operatorname{Proj}(\mathfrak{C})) \approx \mathcal{A}_{e}(\mathfrak{C}) \approx \underline{\operatorname{Sex}}(\mathfrak{C}) \approx \mathcal{B}^{m}(\mathfrak{C}) \approx \mathcal{B}(\mathfrak{C} / \operatorname{Inj}(\mathfrak{C})) .
$$

Proof. The functor $F: \mathcal{A}_{e}(\mathfrak{C}) \rightarrow \underline{\operatorname{Sex}}(\mathfrak{C})$ defined by $F\{A, f, B\}=$ the class of the short exact sequence $0 \rightarrow \operatorname{Ker}(f) \rightarrow A \rightarrow B \rightarrow 0$ and the functor $G: \mathcal{B}_{m}(\mathfrak{C}) \rightarrow \underline{\text { Sex }}(\mathfrak{C})$ defined by $G[A, f, B]=$ the class of the short exact sequence $0 \rightarrow A \rightarrow B \rightarrow \operatorname{Coker}(f) \rightarrow 0$, are obviously equivalences. The last assertion follows from Proposition 3.8 and Corollary 3.10.

Remark 4.11. By the above result, we have a Morita pair $(\mathfrak{C} / \operatorname{Proj}(\mathfrak{C}), \mathfrak{C} / \operatorname{Inj}(\mathfrak{C}))$. Hence if $\mathfrak{C}$ is Frobenius, the stable category $\mathfrak{C} / \operatorname{Proj}(\mathfrak{C})$ is dualizing. More generally if $(\mathfrak{C}, \mathfrak{D})$ is a Morita pair, then the same is true for the pairs $(\mathcal{A}(\mathfrak{C}), \overline{\mathcal{B}(\mathfrak{D})}),(\mathcal{B}(\mathfrak{D}), \overline{\mathcal{A}(\mathfrak{C})})$ of the induced stable categories. Indeed by Corollary 4.10, we have: $\mathcal{A}(\mathcal{A}(\mathfrak{C})) \approx \overline{\mathcal{A}_{e}(\mathcal{A}(\mathfrak{C}))} \approx \mathcal{A}_{e}(\mathcal{B}(\mathfrak{D})) \approx \mathcal{B}^{m}(\mathcal{B}(\mathfrak{D}))$ $\approx \mathcal{B}(\overline{\mathcal{B}(\mathfrak{D})})$. Similarly we have an equivalence: $\overline{\mathcal{A}(\underline{\mathcal{B}(\mathfrak{D})})} \approx \mathcal{B}(\overline{\mathcal{A}(\mathfrak{C})})$.

For any morphism $f: A \rightarrow B$ in $\mathfrak{C}$, let $K_{f}=\operatorname{Ker}(f), I_{f}=\operatorname{Im}(f), C_{f}=\operatorname{Coker}(f), k_{f}=$ $\operatorname{ker}(f), \varepsilon_{f}=\operatorname{coim}(f), i_{f}=i m(f), c_{f}=\operatorname{coker}(f)$. Then the canonical analysis of $f$, induces 
the following functorial exact sequences in $\mathcal{A}(\mathfrak{C})$ and $\mathcal{B}(\mathfrak{C})$ :

$$
\begin{aligned}
& 0 \longrightarrow\left.\longrightarrow A, \varepsilon_{f}, I_{\mathrm{f}}\right\} \stackrel{\left\{1_{A}, i_{f}\right\}}{\longrightarrow}\{A, f, B\} \stackrel{\left\{0, c_{f}\right\}}{\longrightarrow} \mathbf{P}\left(C_{f}\right) \stackrel{\left\{0,1_{C_{f}}\right\}}{\longrightarrow}\left\{B, c_{f}, C_{f}\right\} \longrightarrow 0 \\
& 0 \longrightarrow\left[K_{f}, k_{f}, A\right] \stackrel{\left[1_{K_{f}}, 0\right]}{\longrightarrow} \mathbf{Q}\left(K_{f}\right) \stackrel{\left[k_{f}, 0\right]}{\longrightarrow}[A, f, B] \stackrel{\left[\varepsilon_{f}, 1_{B}\right]}{\longrightarrow}\left[I_{f}, i_{f}, B\right] \longrightarrow 0
\end{aligned}
$$

where $\operatorname{Im}\left(\left\{0, c_{f}\right\}\right)=\left\{I_{f}, i_{f}, B\right\}$ and $\operatorname{Im}\left(\left[k_{f}, 0\right]\right)=\left[A, \varepsilon_{f}, I_{f}\right]$.

Corollary 4.12. The category $\mathcal{A}_{e}(\mathfrak{C})$ is coreflective in $\mathcal{A}(\mathfrak{C})$. If $\mathfrak{C}$ has enough projectives, then $\mathcal{A}_{e}(\mathfrak{C})$ is also reflective. The category $\mathcal{B}^{m}(\mathfrak{C})$ is reflective in $\mathcal{B}(\mathfrak{C})$. If $\mathfrak{C}$ has enough injectives, then $\mathcal{B}^{m}(\mathfrak{C})$ is also coreflective.

Proof. The exact sequences (4.7), (4.8), imply trivially that $\left\{A, \varepsilon_{f}, I_{f}\right\}$ is the coreflection of $\{A, f, B\}$ in $\mathcal{A}_{e}(\mathfrak{C})$ and $\left[I_{f}, i_{f}, B\right]$ is the reflection of $[A, f, B]$ in $\mathcal{B}^{m}(\mathfrak{C})$. Suppose that $\mathfrak{C}$ has enough projectives. If $\{A, f, B\} \in \mathcal{A}(\mathfrak{C})$, let $p: P \rightarrow B$ be an epimorphism with $P$ projective. Then the object $\left\{A \oplus P,{ }^{t}(f, p), B\right\}$ belongs to $\mathcal{A}_{e}(\mathfrak{C})$ and the morphism $\left\{{ }^{t}\left(1_{A}, 0\right), 1_{B}\right\}$ : $\{A, f, B\} \rightarrow\left\{A \oplus P,{ }^{t}(f, p), B\right\}$ is the reflection of $\{A, f, B\}$ in $\mathcal{A}_{e}(\mathfrak{C})$. Dually for $\mathcal{B}^{m}(\mathfrak{C})$.

By Corollary 3.9, the categories involved in Corollary 4.10 are equivalent to the module categories mod- $(\mathfrak{C} / \operatorname{Proj}(\mathfrak{C}))$ and $((\mathfrak{C} / \operatorname{Inj}(\mathfrak{C}))-\bmod )^{o p}$. Hence all these categories are abelian with enough projectives and injectives, and using these equivalences we know their projective and injective objects. In particular if $\mathfrak{C}$ is Frobenius then the same is true for any one of the these categories.

The following result is a relative version of Corollary 4.10.

Proposition 4.13. (1) If $\mathfrak{C}$ is right coherent and $\mathfrak{X}$ is a contravariantly finite in $\mathfrak{C}$, then the sequence of additive categories $0 \rightarrow \mathfrak{X} \hookrightarrow \mathfrak{C} \rightarrow \mathfrak{C} / \mathfrak{X} \rightarrow 0$, induces a short exact sequence of abelian categories: $0 \rightarrow \mathcal{A}(\mathfrak{C} / \mathfrak{X}) \hookrightarrow \mathcal{A}(\mathfrak{C}) \rightarrow \mathcal{A}(\mathfrak{X}) \rightarrow 0$.

(2) If $\mathfrak{C}$ is left coherent and $\mathfrak{X}$ is covariantly finite in $\mathfrak{C}$, then the sequence of additive categories $0 \rightarrow \mathfrak{X} \hookrightarrow \mathfrak{C} \rightarrow \mathfrak{C} / \mathfrak{X} \rightarrow 0$, induces a short exact sequence of abelian categories: $0 \rightarrow \mathcal{B}(\mathfrak{C} / \mathfrak{X}) \hookrightarrow \mathcal{B}(\mathfrak{C}) \rightarrow \mathcal{B}(\mathfrak{X}) \rightarrow 0$.

Proof. (1) If $\mathfrak{C}$ is skeletally small, then using functor categories the result follows from [7]. We sketch a different proof in the general case. Since $\mathfrak{X}$ is contravariantly finite in $\mathfrak{C}$ and $\mathfrak{C}$ is right coherent it follows easily that $\mathfrak{X}$ and $\mathfrak{C} / \mathfrak{X}$ are right coherent. Hence $\mathcal{A}(\mathfrak{C}), \mathcal{A}(\mathfrak{X})$ and $\mathcal{A}(\mathfrak{C} / \mathfrak{X})$ are abelian. Let $A \in \mathfrak{C}$; then using the existence of weak kernels and the contravariant finiteness of $\mathfrak{X}$ in $\mathfrak{C}$, one can construct an $\mathfrak{X}$-exact complex $X_{A}^{1} \stackrel{x_{A}^{1}}{\longrightarrow} X_{A}^{0} \stackrel{x_{A}^{0}}{\longrightarrow} A \rightarrow 0$ (i.e. the complex becomes exact when we apply to it the functor $\mathfrak{C}(X,-)$, for any $X \in \mathfrak{X})$. Using this complex, define a functor $F_{0}: \mathfrak{C} \rightarrow \mathcal{A}(\mathfrak{X})$, by $F_{0}(A)=\left\{X_{A}^{1}, x_{A}^{1}, X_{A}^{0}\right\}$. Since $\mathcal{A}(\mathfrak{X})$ is abelian, by the universal property of $\mathcal{A}(\mathfrak{C})$, there exists a unique right exact functor $F: \mathcal{A}(\mathfrak{C}) \rightarrow \mathcal{A}(\mathfrak{X})$ with $F \mathbf{P}_{\mathfrak{C}}=F_{0}$. It is not difficult to see that $F_{0}$ sends weak kernel sequences in $\mathfrak{C}$ to exact sequences in $\mathcal{A}(\mathfrak{X})$, hence by Proposition $4.5, F$ is exact. Moreover $F G=\operatorname{Id}_{\mathcal{A}(\mathfrak{X})}$, where $G: \mathcal{A}(\mathfrak{X}) \rightarrow \mathcal{A}(\mathfrak{C})$ is the right exact functor induced by the inclusion $\mathfrak{X} \hookrightarrow \mathfrak{C}$. Then $\mathcal{A}(\mathfrak{C}) / \operatorname{Ker} F \approx \mathcal{A}(\mathfrak{X})$ by $[\mathbf{7}]$. Finally we see easily that $\operatorname{Ker} F$ is the full subcategory of $\mathcal{A}(\mathfrak{C})$ consisting of the $\mathfrak{X}$-epics in $\mathfrak{C}$. By Corollary 4.10, $\operatorname{Ker} F$ is equivalent to $\mathcal{A}(\mathfrak{C} / \mathfrak{X})$. Part (2) follows by duality.

Corollary 4.14. Let $\mathfrak{C}$ be a right Morita (left Morita) category and let $\mathfrak{X}$ be a contravariantly (covariantly) finite subcategory of $\mathfrak{C}$. Then $\mathfrak{X}$ and the stable category $\mathfrak{C} / \mathfrak{X}$ are right Morita (left Morita) categories.

Proof. If $\mathfrak{C}$ be a right Morita and $\mathfrak{X}$ is contravariantly finite then $\mathfrak{X}$ is right coherent. Since $\mathcal{A}(\mathfrak{C})$ has injectives, and since by the previous Proposition $\mathcal{A}(\mathfrak{C} / \mathfrak{X})$ is a localizing subcategory of $\mathcal{A}(\mathfrak{C})$ with quotient $\mathcal{A}(\mathfrak{X})$, we have that $\mathcal{A}(\mathfrak{X})$ has injectives. If $\mathfrak{C}$ is left Morita and $\mathfrak{X}$ is covariantly finite then $\mathfrak{C}^{o p}$ is right Morita and $\mathfrak{X}^{o p}$ is contravariantly finite in $\mathfrak{C}^{o p}$. Hence $\mathfrak{X}^{o p}$ is right Morita and consequently $\mathfrak{X}$ is left Morita. The case of $\mathfrak{C} / \mathfrak{X}$ is left to the reader. 
We close this section studying when a Freyd category is a module category. We recall that an object $A \in \mathfrak{C}$ is called compact $[\mathbf{3 6}]$ if the functor $\mathfrak{C}(A,-): \mathfrak{C} \rightarrow \mathfrak{A} b$ preserves all small coproducts. An object $U \in \mathfrak{C}$ is called a split generator if for any morphism $f: A \rightarrow B$ in $\mathfrak{C}$, $\mathfrak{C}(U, f)$ is epic implies that $f$ is split epic.

Proposition 4.15. For an additive category $\mathfrak{C}$, the following are equivalent:

(1) $\mathcal{A}(\mathfrak{C})$ is a (functor) module category.

(2) $\mathfrak{C}$ is right coherent with coproducts and a (set of) compact split generator $(s)$.

If (1) or (2) is true, then there exists a ring $\Lambda$ and an equivalence $\mathfrak{C} \approx \operatorname{Proj}(\operatorname{Mod}-\Lambda)$.

Proof. (1) $\Rightarrow(2)$ If there exists an equivalence $\mathcal{A}(\mathfrak{C}) \approx \operatorname{Mod}-\mathcal{U}$, then we have trivially $\mathfrak{C} \approx$ $\operatorname{Proj}(\operatorname{Mod}-\mathcal{U})$. Hence the properties described in $(2)$ are true.

$(2) \Rightarrow(1)$ The right coherence of $\mathfrak{C}$ implies that $\mathcal{A}(\mathfrak{C})$ is abelian. Since $\mathfrak{C}$ has coproducts, the same is true for $\mathcal{A}(\mathfrak{C})$ (the naive construction of coproducts in $\mathcal{A}(\mathfrak{C})$ works). Hence $\mathcal{A}(\mathfrak{C})$ is a cocomplete abelian category. If $\mathcal{U}$ is a set of compact objects in $\mathfrak{C}$, then $\mathbf{P}(\mathcal{U})$ is a set of compact projective objects in $\mathcal{A}(\mathfrak{C})$. It is easy to see that if moreover $\mathcal{U}$ consists of split generators, then $\mathbf{P}(\mathcal{U})$ consists of generators in $\mathcal{A}(\mathfrak{C})$. By [19], we have an equivalence $\mathcal{A}(\mathfrak{C}) \approx \operatorname{Mod}-\mathcal{U}$.

\section{Tensor Products}

We fix objects $\{A, f, B\} \in \mathcal{A}(\mathfrak{C})$ and $[C, g, D] \in \mathcal{B}(\mathfrak{C})$, where $\mathfrak{C}$ is any additive category. For any $X \in \mathfrak{C}$, setting $F_{[C, g, D]}(X)=\operatorname{Coker}(g, X)$ and $F_{\{A, f, B\}}(X)=\operatorname{Coker}(X, f)$, we obtain additive functors $F_{[C, g, D]}: \mathfrak{C} \rightarrow \mathfrak{A} b$ and $F_{\{A, f, B\}}: \mathfrak{C}^{o p} \rightarrow \mathfrak{A} b$. By the universal property of $\mathcal{A}(\mathfrak{C})$, there exists a unique cokernel preserving functor $F_{[C, g, D]}^{!}: \mathcal{A}(\mathfrak{C}) \rightarrow \mathfrak{A} b$ with $F_{[C, g, D]}^{!} \mathbf{P}_{\mathfrak{C}}=$ $F_{[C, g, D]}$. By the universal property of $\mathcal{B}(\mathfrak{C})$, there exists a unique functor converting kernels to cokernels, $F_{\{A, f, B\}}^{*}: \mathcal{B}(\mathfrak{C})^{o p} \rightarrow \mathfrak{A} b$ with $F_{\{A, f, B\}}^{*} \mathbf{Q}_{\mathfrak{C}}=F_{\{A, f, B\}}$. We set

$$
\begin{aligned}
F_{[C, g, D]}^{!} & :=-\otimes_{\mathfrak{C}}[C, g, D]: \mathcal{A}(\mathfrak{C}) \longrightarrow \mathfrak{A} b, \\
F_{\{A, f, B\}}^{*} & :=\{A, f, B\} \otimes_{\mathfrak{C}}-: \mathcal{B}(\mathfrak{C})^{o p} \longrightarrow \mathfrak{A} b .
\end{aligned}
$$

In this way we obtain a tensor product bifunctor $-\otimes_{\mathfrak{C}}-: \mathcal{A}(\mathfrak{C}) \times \mathcal{B}(\mathfrak{C})^{o p} \rightarrow \mathfrak{A} b$ between the Freyd categories $\mathcal{A}(\mathfrak{C}), \mathcal{B}(\mathfrak{C})$ and by definition we have:

$$
\begin{aligned}
\{A, f, B\} \otimes_{\mathfrak{C}}[C, g, D] & =\operatorname{Coker}(\operatorname{Coker}(D, f) \rightarrow \operatorname{Coker}(C, f)) \\
& =\operatorname{Coker}(\operatorname{Coker}(g, A) \rightarrow \operatorname{Coker}(g, B)) .
\end{aligned}
$$

Remark 5.1. In case $\mathfrak{C}$ is (skeletally) small and $\mathfrak{D}$ is cocomplete abelian, then using the fact that in Mod-C, $\mathfrak{C}-$ Mod any object is a direct limit of finitely presented objects, and also using the equivalences mod- $\mathfrak{C} \approx \mathcal{A}(\mathfrak{C}), \mathfrak{C}-\bmod \approx \mathcal{B}(\mathfrak{C})^{o p}$, one can define easily the tensor product functors $-\otimes_{\mathfrak{C}}-:\left[\mathfrak{C}^{o p}, \mathfrak{D}\right] \times \mathfrak{C}-\operatorname{Mod} \rightarrow \mathfrak{D}$ and $-\otimes_{\mathfrak{C}}-: \operatorname{Mod}-\mathfrak{C} \times[\mathfrak{C}, \mathfrak{D}] \rightarrow \mathfrak{D}[\mathbf{1 9}]$. Conversely the tensor product of $[\mathbf{1 9}]$ in case $\mathfrak{D}=\mathfrak{A} b$, restricted to finitely presented functors coincide with the tensor product of the Freyd categories constructed above.

We are interested in case the Freyd categories $\mathcal{A}(\mathfrak{C}), \mathcal{B}(\mathfrak{C})$ are abelian. So from now on we assume that the additive category $\mathfrak{C}$ is coherent. In this case $\forall\{A, f, B\} \in \mathcal{A}(\mathfrak{C}), \forall[C, g, D] \in$ $\mathcal{B}(\mathfrak{C})$ the derived functors

$$
\begin{gathered}
\mathcal{T}_{o r_{i}^{\mathfrak{C}}}(-,[C, g, D])=\mathcal{L}_{i}\left(-\otimes_{\mathfrak{C}}[C, g, D]\right): \mathcal{A}(\mathfrak{C}) \longrightarrow \mathfrak{A} b, \\
\mathcal{T} \operatorname{or}_{i}^{\mathfrak{C}}(\{A, f, B\},-)=\mathcal{L}_{i}\left(\{A, f, B\} \otimes_{\mathfrak{C}}-\right): \mathcal{B}(\mathfrak{C})^{o p} \longrightarrow \mathfrak{A} b
\end{gathered}
$$

are defined, and is easy to see that the bifunctor $-\otimes_{\mathfrak{C}}-$ is left balanced, hence the bifunctor $\mathcal{T}$ or $_{i}^{\mathfrak{C}}(-,-): \mathcal{A}(\mathfrak{C}) \times \mathcal{B}(\mathfrak{C})^{o p} \rightarrow \mathfrak{A} b, \forall i \geqslant 0$, is defined. The flat objects (i.e. the $-\otimes_{\mathfrak{C}}-$ acyclic objects) in $\mathcal{A}(\mathfrak{C}), \mathcal{B}(\mathfrak{C})$ are obviously the projectives, injectives objects respectively. 
Lemma 5.2. There are functorial morphisms

$$
\psi:-\otimes_{\mathfrak{C}} \mathbf{Q}^{!}(?) \rightarrow \mathcal{A}(\mathfrak{C})(?,-), \quad \chi: \mathbf{P}^{*}(?) \otimes_{\mathfrak{C}}-\rightarrow \mathcal{B}(\mathfrak{C})(-, ?)
$$

with $\operatorname{Coker}(\psi)=\underline{\mathcal{A}(\mathfrak{C})}(?,-), \operatorname{Coker}(\chi)=\overline{\mathcal{B}(\mathfrak{C})}(-, ?)$, inducing isomorphisms

$$
\begin{aligned}
& -\otimes_{\mathfrak{C}} \mathbf{Q}(?) \cong \mathcal{A}(\mathfrak{C})(\mathbf{P}(?),-), \quad \mathbf{P}(?) \otimes_{\mathfrak{C}}-\cong \mathcal{B}(\mathfrak{C})(-, ?),
\end{aligned}
$$

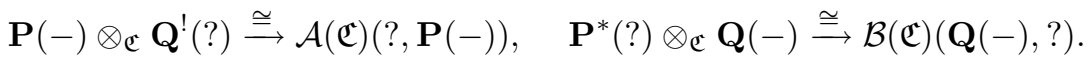

Proof. The proof is standard and is left to the reader.

Theorem 5.3. There are functorial isomorphisms

$$
\mathcal{T}_{\text {or }}^{\mathfrak{C}}(\operatorname{Tr}(-), ?) \stackrel{\cong}{\mathcal{B}(\mathfrak{C})}(?,-), \quad \mathcal{T}_{\text {or }}^{\mathfrak{C}}(-, \operatorname{Tr}(?)) \stackrel{\cong}{\mathcal{A}(\mathfrak{C})}(?,-) .
$$

Proof. We prove only the second isomorphism. Let $\{A, f, B\} \in \mathcal{A}(\mathfrak{C})$ and let $h: B \rightarrow X$ be a weak cokernel of $f$. Then we have an injective resolution of $\operatorname{Tr}\{A, f, B\}$ in $\mathcal{B}(\mathfrak{C}): 0 \rightarrow$ $\operatorname{Tr}\{A, f, B\} \rightarrow \mathbf{Q}(A) \stackrel{\mathbf{Q}(f)}{\longrightarrow} \mathbf{Q}(B) \stackrel{\mathbf{Q}(h)}{\longrightarrow} \mathbf{Q}(X) \rightarrow \cdots$. If $\{C, g, D\} \in \mathcal{A}(\mathfrak{C})$, then the abelian group $\mathcal{T}_{\text {or }_{1}^{\mathfrak{C}}}(\{C, g, D\}, \operatorname{Tr}\{A, f, B\})$ is the homology of the complex $\{C, g, D\} \otimes_{\mathfrak{C}} \mathbf{Q}(X) \rightarrow$ $\{C, g, D\} \otimes_{\mathfrak{C}} \mathbf{Q}(B) \rightarrow\{C, g, D\} \otimes_{\mathfrak{C}} \mathbf{Q}(A)$. But by Lemma 5.2 this complex is isomorphic to the complex $\mathcal{A}(\mathfrak{C})(\mathbf{P}(X),\{C, g, D\}) \stackrel{h^{*}}{\longrightarrow} \mathcal{A}(\mathfrak{C})(\mathbf{P}(B),\{C, g, D\}) \stackrel{f^{*}}{\longrightarrow} \mathcal{A}(\mathfrak{C})(\mathbf{P}(A),\{C, g, D\})$. Let $\{0, b\}: \mathbf{P}(B) \rightarrow\{C, g, D\}$ be in $\operatorname{Ker}\left(f^{*}\right)$. Then there exists $a: A \rightarrow C$ with $a \circ g=f \circ b$. Hence we obtain a morphism $\{a, b\}:\{A, f, B\} \rightarrow\{C, g, D\}$, which obviously is uniquely determined by $b$. If $\{0, b\}$ is in $\operatorname{Im}\left(h^{*}\right)$, then there exists $m: X \rightarrow D$ with $\{0, h \circ m\}=\{0, b\}$. This means that $b-h \circ m=t \circ g$. Then the morphism $\{a, b\}:\{A, f, B\} \rightarrow\{C, g, D\}$ factors through the projective $\mathbf{P}(X)$. Indeed $\{a, b\}=\{0, h\} \circ\{0, m\}$ where $\{0, h\}:\{A, f, B\} \rightarrow$ $\mathbf{P}(X)$ and $\{0, m\}: \mathbf{P}(X) \rightarrow\{C, g, D\}$. Thus we can define a morphism $\operatorname{Ker}\left(f^{*}\right) / \operatorname{Im}\left(h^{*}\right) \rightarrow$ $\mathcal{A}(\mathfrak{C})(\{A, f, B\},\{C, g, D\})$ in $\mathfrak{A} b$. We leave to the reader the easy demonstration that the above morphism is indeed an isomorphism.

Corollary 5.4. $\forall\{A, f, B\} \in \mathcal{A}(\mathfrak{C}), \forall[C, g, D] \in \mathcal{B}(\mathfrak{C})$, there are isomorphisms:

$$
\underline{\mathcal{A}(\mathfrak{C})}(\{A, f, B\},\{C, g, D\}) \stackrel{\cong}{\mathcal{T}_{o r}}{ }_{1}^{\mathfrak{C}}(\{A, f, B\},[C, g, D]) \stackrel{\cong}{\mathcal{B}(\mathfrak{C})}([A, f, B],[C, g, D]) .
$$

Let $\Omega$ be the syzygy functor in $\mathcal{A}(\mathfrak{C})$ and $\Sigma$ be the cosyzygy functor in $\overline{\mathcal{B}\left(\mathfrak{C}^{2}\right)}$.

Proposition 5.5. (1) There is an exact sequence

$$
0 \longrightarrow \underline{\mathcal{A}(\mathfrak{C})}(?, \Omega(-)) \longrightarrow-\otimes_{\mathfrak{C}} \mathbf{Q}^{!}(?) \stackrel{\psi}{\longrightarrow} \mathcal{A}(\mathfrak{C})(?,-) \longrightarrow \underline{\mathcal{A}(\mathfrak{C})}(?,-) \longrightarrow 0
$$

and functorial isomorphisms $\mathcal{T}_{\text {or }}^{\mathfrak{C}}{ }_{i+1}^{\mathfrak{r}}\left(-, \mathbf{Q}^{!}(?)\right) \cong \underline{\mathcal{A}(\mathfrak{C})}\left(?, \Omega^{i+2}(-)\right), \quad \forall i \geqslant 0$.

(2) There is an exact sequence

$$
0 \longrightarrow \overline{\mathcal{B}(\mathfrak{C})}(\Sigma(-), ?) \longrightarrow \mathbf{P}^{*}(?) \otimes_{\mathfrak{C}}-\stackrel{\chi}{\longrightarrow} \mathcal{B}(\mathfrak{C})(-, ?) \longrightarrow \overline{\mathcal{B}(\mathfrak{C})}(-, ?) \longrightarrow 0
$$

and functorial isomorphisms $\mathcal{T}_{\text {or }}^{\mathfrak{C}}\left(\mathbf{P}^{*}(-), ?\right) \stackrel{\cong}{\mathcal{B}(\mathfrak{C})}\left(\Sigma^{i+2}(?),-\right), \quad \forall i \geqslant 0$.

(3) There are exact sequences

$$
\begin{gathered}
0 \longrightarrow \mathcal{E} x t_{\mathcal{A}(\mathfrak{C})}^{1}(\operatorname{Tr}(-), ?) \longrightarrow-\otimes_{\mathfrak{C}} ? \longrightarrow \mathcal{A}\left(\mathfrak{C}^{\mathfrak{c}}\right)\left(\mathbf{P}^{*}(-), ?\right) \longrightarrow \mathcal{E} x t_{\mathcal{A}(\mathfrak{C})}^{2}(\operatorname{Tr}(-), ?) \longrightarrow 0 \\
0 \longrightarrow \mathcal{E} x t_{\mathcal{B}(\mathfrak{C})}^{1}(-, \operatorname{Tr}(?)) \longrightarrow ? \otimes_{\mathfrak{C}}-\longrightarrow \mathcal{B}(\mathfrak{C})\left(-, \mathbf{Q}^{!}(?)\right) \rightarrow \mathcal{E} x t_{\mathcal{B}(\mathfrak{C})}^{2}(-, \operatorname{Tr}(?)) \longrightarrow 0
\end{gathered}
$$

Proof. (1) If $X:=\{A, f, B\}, Y:=\{C, g, D\} \in \mathcal{A}(\mathfrak{C})$, by Theorem 5.3 we have:

$$
\underline{\mathcal{A}(\mathfrak{C})}\left(Y, \Omega^{2}(X)\right) \cong \mathcal{T} \text { or }_{1}^{\mathfrak{C}}\left(\Omega^{2}(X), \operatorname{Tr}(Y)\right) \cong \mathcal{T} \text { or }_{3}^{\mathfrak{C}}(X, \operatorname{Tr}(Y)) \cong \mathcal{T}_{\text {or }}^{\mathfrak{C}}\left(X, \Sigma^{2} \operatorname{Tr}(Y)\right)
$$

Since $\Sigma^{2} \operatorname{Tr}(Y)=\mathbf{Q}^{!}(Y)$, we have an isomorphism $\mathcal{A}(\mathfrak{C})\left(Y, \Omega^{2}(X)\right) \cong \mathcal{T}$ or ${ }_{1}^{\mathfrak{C}}\left(X, \mathbf{Q}^{!}(Y)\right)$. By induction and dimension-shifting we get the isomorphism of part (1). Applying the functor 
- $\otimes_{\mathfrak{C}} \mathbf{Q}^{!}(Y)$ to the projective presentation $0 \rightarrow \Omega(X) \rightarrow \mathbf{P}(B) \rightarrow X \rightarrow 0$ and using Lemma 5.2 , we have the commutative diagram

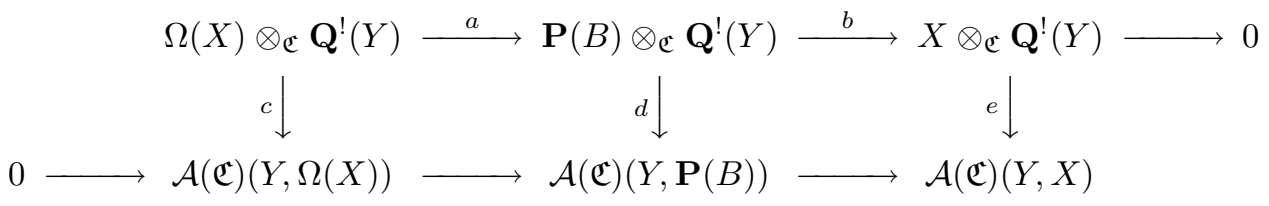

where the vertical morphisms are the natural morphisms of Lemma 5.2; in particular $d$ is an isomorphism. Then using the snake Lemma and Lemma 5.2, we have: $\operatorname{Ker}(a) \cong \operatorname{Ker}(c) \cong$ $\mathcal{T}_{\text {or }}^{\mathfrak{C}}\left(X, \mathbf{Q}^{!}(Y)\right), \operatorname{Ker}(e) \cong \operatorname{Coker}(c) \cong \mathcal{A}(\mathfrak{C})(Y, \Omega(X))$. From these isomorphisms it follows that for the natural morphisms $\psi_{X, Y}: X \otimes_{\mathfrak{C}} \mathbf{Q}^{!}(Y) \rightarrow \mathcal{A}(\mathfrak{C})(Y, X)$ and $\psi_{\Omega(X), Y}:$ $\Omega(X) \otimes_{\mathfrak{C}} \mathbf{Q}^{!}(Y) \rightarrow \mathcal{A}(\mathfrak{C})(Y, \Omega(X))$ of Lemma 5.2 , we have: $\operatorname{Ker}\left(\psi_{X, Y}\right) \cong \mathcal{A}(\mathfrak{C})(Y, \Omega(X))$ and $\operatorname{Ker}\left(\psi_{\Omega(X), Y}\right) \cong \mathcal{A}(\mathfrak{C})\left(Y, \Omega^{2}(X)\right)$. The desired exact sequence follows from the previous Lemma. The proof of part (2) is dual and part (3) is similar to (1).

Corollary 5.6. If $\mathfrak{C}$ is a coherent category, then: $\operatorname{gl} \operatorname{dim} \mathcal{A}(\mathfrak{C})=\operatorname{gl} \cdot \operatorname{dim} \mathcal{B}(\mathfrak{C})$.

Proof. Suppose first that gl. $\operatorname{dim} \mathcal{B}(\mathfrak{C})=n<\infty$. Then $\mathcal{T}_{\text {or }}^{\mathfrak{C}}{ }_{n+1}^{\mathfrak{C}}(-,-)=0$, and by Proposition 5.5 we have, $\forall X \in \mathcal{A}(\mathfrak{C})$ :

$$
0=\mathcal{T} \text { or }{ }_{n+1}^{\mathfrak{C}}\left(X, \mathbf{Q}^{!} \Omega^{n+2}(X)\right) \cong \underline{\mathcal{A}(\mathfrak{C})}\left(\Omega^{n+2}(X), \Omega^{n+2}(X)\right) .
$$

So $\Omega^{n+2}(X)$ is projective and this means gl. $\operatorname{dim} \mathcal{A}(\mathfrak{C})=m \leqslant n+1$. Suppose that $m=n+1$. Then there exists $X \in \mathcal{A}(\mathfrak{C})$ with $\Omega^{n+1}(X)$ non projective. Hence $\mathcal{A}(\mathfrak{C})\left(\Omega^{n+1}(X), \Omega^{n+1}(X)\right) \neq$

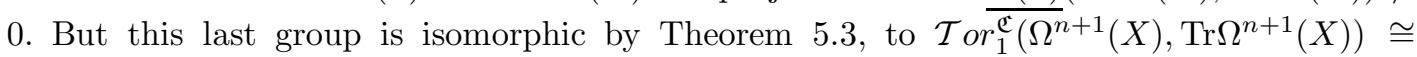
$\mathcal{T}$ or ${ }_{n+2}^{\mathfrak{C}}\left(X, \operatorname{Tr} \Omega^{n+1}(X)\right)$ which is trivial since gl.dimB(C) $(\mathfrak{C})=n$. Hence $m \neq n+1$. We conclude that gl.dim $\mathcal{A}(\mathfrak{C})=m \leqslant n=$ gl.dim $\mathcal{B}(\mathfrak{C})$. By duality gl.dim $\mathcal{B}(\mathfrak{C}) \leqslant \operatorname{gl} \cdot \operatorname{dim} \mathcal{A}(\mathfrak{C})$. Hence gl.dim $\mathcal{A}(\mathfrak{C})=$ gl.dim $\mathcal{B}(\mathfrak{C})$. This completes the proof observing that by the above arguments gl.dim $\mathcal{A}(\mathfrak{C})=\infty \Leftrightarrow \operatorname{gl} . \operatorname{dim} \mathcal{B}(\mathfrak{C})=\infty$.

Remark 5.7. (1) In case $\mathfrak{C}$ is a skeletally small coherent additive category, then Corollary 5.6 shows that gl.dimmod $-\mathfrak{C}=$ gl.dim $\mathfrak{C}-\bmod =$ the weak dimension of $\mathfrak{C}$. By $[\mathbf{4 6}]$, gl.dimmod $-\mathfrak{C}$ is also the FP-dimension of Mod-C .

(2) If Mod-C $\mathfrak{C}$ and $\mathfrak{C}-$ Mod are locally Noetherian [24], then we have the analogue of the classical result of Auslander: gl.dimMod- $\mathfrak{C}=\operatorname{gl}$.dim $\mathfrak{C}-$ Mod since then $\mathfrak{C}$ is coherent and the finitely generated left and right $\mathfrak{C}$ modules coincide with the finitely presented ones.

(3) By Theorem 5.3, for any morphism $f$ in $\mathfrak{C}$ we have:

$$
\begin{aligned}
& \mathcal{T} \operatorname{or}_{1}^{\mathfrak{C}}(?, \operatorname{Coker}(f,-)) \cong \underline{\bmod }-\mathfrak{C}(\operatorname{Coker}(-, f), ?) \\
& \mathcal{T}_{\operatorname{cor}}^{\mathfrak{C}}(\operatorname{Coker}(-, f), ?) \cong \mathfrak{C}-\underline{\bmod }(\operatorname{Coker}(f,-), ?) .
\end{aligned}
$$

¿From the proof of this result it is not difficult to see that the above formulas are true in Mod $-\mathfrak{C}$ if ? is not necessarily finitely presented, and $\mathfrak{C}$ not necessarily coherent. If we denote by D : Mod-C $\rightarrow \mathfrak{C}-$ Mod the character module functor defined by $\mathrm{D}(F)=\mathfrak{A} b(F-, \mathbb{Q} / \mathbb{Z})$, then for any $F \in \bmod -\mathfrak{C}$, we have the formulas

$$
\begin{aligned}
& \mathcal{T}_{\text {or }}{ }_{1}^{\mathfrak{C}}(-, \operatorname{Tr} F) \cong \underline{\operatorname{Mod}}-\mathfrak{C}(F,-), \quad \mathcal{T} \text { or }{ }_{1}^{\mathfrak{C}}(\operatorname{Tr} F,-) \cong \mathfrak{C}-\underline{\operatorname{Mod}}(F,-), \\
& \mathcal{E} x t_{\operatorname{Mod}-\mathfrak{C}}^{1}(-, \mathrm{DTr} F) \cong \mathrm{DMod}-\mathfrak{C}(F,-), \quad \mathrm{DE} x t_{\operatorname{Mod}-\mathfrak{C}}^{1}(F,-) \cong \overline{\operatorname{Mod}}-\mathfrak{C}(-, \mathrm{D} \operatorname{Tr} F) .
\end{aligned}
$$

\section{Free Abelian and Auslander Categories}

Suppose that $\mathfrak{C}$ is an additive category. A free abelian category over $\mathfrak{C}$ is a pair $(F, \mathcal{F})$ consisting of an abelian category $\mathcal{F}$, and an additive functor $F: \mathfrak{C} \rightarrow \mathcal{F}$, satisfying the following universal property: 
$(\dagger)$ for any additive functor $G: \mathfrak{C} \rightarrow \mathfrak{D}$ to an abelian category $\mathfrak{D}$, there exists a unique exact functor $G^{\diamond}: \mathcal{F} \rightarrow \mathfrak{D}$, such that: $G^{\diamond} F=G$.

Theorem 6.1. Let $\mathfrak{C}$ be an additive category.

(1) The Freyd categories $\mathcal{A B}(\mathfrak{C}), \mathcal{B A}(\mathfrak{C})$ are abelian with enough projectives and injectives, and the pair $(\mathcal{B}(\mathfrak{C}), \mathcal{A}(\mathfrak{C}))$ is Morita, hence there is an equivalence:

$$
\mathcal{A B}(\mathfrak{C}) \stackrel{\approx}{\sim} \mathcal{B A}(\mathfrak{C})
$$

(2) If $\mathcal{F}(\mathfrak{C}):=\mathcal{A B}(\mathfrak{C})$, then gl.dim $\mathcal{F}(\mathfrak{C})=0$ or 2 , and the first alternative appears iff $\mathfrak{C}$ is semisimple abelian, in which case: $\mathfrak{C} \approx \mathcal{F}(\mathfrak{C})$.

(3) $\mathcal{F}\left(\mathfrak{C}^{o p}\right) \approx \mathcal{F}(\mathfrak{C})^{o p}$.

(4) $\mathcal{F}(\mathfrak{C})$ is the free abelian category over $\mathfrak{C}$. Every abelian category is a Gabriel-quotient of the free abelian category over its underling additive category.

(5) The additive category $\mathfrak{C}$ is fully imbedded in $\mathcal{F}(\mathfrak{C})$ as the full subcategory of projectiveinjective objects of $\mathcal{F}(\mathfrak{C})$, via the functors $\mathbf{P}_{\mathcal{B}(\mathfrak{C})} \mathbf{Q}_{\mathfrak{C}}, \mathbf{Q}_{\mathcal{A}(\mathfrak{C})} \mathbf{P}_{\mathfrak{C}}$.

Proof. Consider the full imbeddings:

$$
\begin{gathered}
\mathbf{P}_{\mathfrak{C}}: \mathfrak{C} \rightarrow \mathcal{A}(\mathfrak{C}), \quad \mathbf{Q}_{\mathfrak{C}}: \mathfrak{C} \rightarrow \mathcal{B}(\mathfrak{C}), \\
\mathbf{P}_{\mathcal{B}(\mathfrak{C})}: \mathcal{B}(\mathfrak{C}) \rightarrow \mathcal{A B}(\mathfrak{C}), \quad \mathbf{Q}_{\mathcal{A}(\mathfrak{C})}: \mathcal{A}(\mathfrak{C}) \rightarrow \mathcal{B A}(\mathfrak{C}) .
\end{gathered}
$$

¿From section $4, \mathcal{A B}(\mathfrak{C}), \mathcal{B A}(\mathfrak{C})$ are abelian with enough projectives and injectives. If $F$ : $\mathfrak{C} \rightarrow \mathfrak{D}$ is an additive functor to an abelian category, then by section 3 there exists a unique kernel preserving functor $F^{*}: \mathcal{B}(\mathfrak{C}) \rightarrow \mathfrak{D}$, with $F^{*} \mathbf{Q}_{\mathfrak{C}}=F$, and a unique cokernel preserving functor $\left(F^{*}\right)^{!}: \mathcal{A B}(\mathfrak{C}) \rightarrow \mathfrak{D}$ with $\left(F^{*}\right)^{!} \mathbf{P}_{\mathcal{B}(\mathfrak{C})}=F^{*}$. Then $\left(F^{*}\right)^{!} \mathbf{P}_{\mathcal{B}(\mathfrak{C})} \mathbf{Q}_{\mathfrak{C}}=F$. Since $F^{*}$ preserves kernels, obviously $\left(F^{*}\right)^{!}$is exact. Let $G: \mathcal{A B}(\mathfrak{C}) \rightarrow \mathfrak{D}$ be another exact functor with $G \mathbf{P}_{\mathcal{B}(\mathfrak{C})} \mathbf{Q}_{\mathfrak{C}}=F$. Then since $\mathbf{P}_{\mathcal{B}(\mathfrak{C})}$ preserves kernels, by the universal property of $\mathbf{Q}_{\mathfrak{C}}$, we have $G \mathbf{P}_{\mathcal{B}(\mathfrak{C})}=F^{*}$, hence $\left(G \mathbf{P}_{\mathcal{B}(\mathfrak{C})}\right)^{!}=\left(F^{*}\right)^{!}$. Since $G$ is exact we can see easily that $\left(G \mathbf{P}_{\mathcal{B}(\mathfrak{C})}\right) !\{A, f, B\}=\operatorname{Coker}\left(G \mathbf{P}_{\mathcal{B}(\mathfrak{C})}(f)\right)=G \operatorname{Coker}\left(\mathbf{P}_{\mathcal{B}(\mathfrak{C})}(f)\right)=G\{A, f, B\}, \forall\{A, f, B\} \in$ $\mathcal{A B}(\mathfrak{C})$, so $\left(F^{*}\right)^{!}=\left(G \mathbf{P}_{\mathcal{B}(\mathfrak{C})}\right)^{!}=G$. We conclude that the pair $\left(\mathbf{P}_{\mathcal{B}(\mathfrak{C})} \mathbf{Q}_{\mathfrak{C}}, \mathcal{A B}(\mathfrak{C})\right)$ is the free abelian category over $\mathfrak{C}$. A similar argument shows that the pair $\left(\mathbf{Q}_{\mathcal{A}(\mathfrak{C})} \mathbf{P}_{\mathfrak{C}}, \mathcal{B A}(\mathfrak{C})\right)$ is the free abelian category over $\mathfrak{C}$. Hence there exists a unique equivalence $D: \mathcal{A B}(\mathfrak{C}) \rightarrow$ $\mathcal{B A}(\mathfrak{C})$, with $D \mathbf{P}_{\mathcal{B}(\mathfrak{C})} \mathbf{Q}_{\mathfrak{C}}=\mathbf{Q}_{\mathcal{A}(\mathfrak{C})} \mathbf{P}_{\mathfrak{C}}$. In particular the pair $(\mathcal{B}(\mathfrak{C}), \mathcal{A}(\mathfrak{C}))$ is Morita. We set $\mathcal{F}(\mathfrak{C}):=\mathcal{A B}(\mathfrak{C})$. Since $\mathcal{B}(\mathfrak{C})$ has kernels gl.dim $\mathcal{F}(\mathfrak{C}) \leqslant 2$. If gl.dim $\mathcal{F}(\mathfrak{C})=1$, then any kernel in $\mathcal{B}(\mathfrak{C})$ splits by Proposition 4.5. In particular $\left[1_{A}, 0\right]:[A, f, B] \rightarrow \mathbf{Q}_{\mathfrak{C}}(A)$ splits. As we have seen this implies that $\mathbf{Q}_{\mathfrak{C}}, \mathbf{P}_{\mathfrak{C}}$ are equivalences. Hence $\mathfrak{C} \approx \mathcal{F}(\mathfrak{C})$ is semisimple abelian, and this is not the case. Thus gl.dim $\mathcal{F}(\mathfrak{C}) \neq 1$. By Proposition 4.5, gl.dim $\mathcal{F}(\mathfrak{C})=0$ iff $\mathfrak{C}$ is semisimple abelian in which case $\mathfrak{C} \approx \mathcal{F}(\mathfrak{C})$. Using Proposition 3.6, we have: $\mathcal{F}\left(\mathfrak{C}^{\circ o p}\right)=$ $\mathcal{A B}\left(\mathfrak{C}^{o p}\right) \approx \mathcal{A}\left(\mathcal{A}(\mathfrak{C})^{o p}\right) \approx \mathcal{B}(\mathcal{A}(\mathfrak{C}))^{o p}=\mathcal{F}(\mathfrak{C})^{o p}$. Let now $\mathfrak{C}$ be abelian. Then we have the adjoint pairs $\left(\mathbf{Q}_{\mathfrak{C}}, \Psi_{\mathfrak{C}}\right),\left(\Phi_{\mathcal{B}(\mathfrak{C})}, \mathbf{P}_{\mathcal{B}(\mathfrak{C})}\right)$. Since $\Psi_{\mathfrak{C}}, \Phi_{\mathcal{B}(\mathfrak{C})}$ are exact and $\mathbf{Q}_{\mathfrak{C}}, \mathbf{P}_{\mathcal{B}(\mathfrak{C})}$ are fully faithful, $\operatorname{Ker} \Phi_{\mathcal{B}(\mathfrak{C})}$ is a localizing subcategory of $\mathcal{F}(\mathfrak{C}), \operatorname{Ker} \Psi_{\mathfrak{C}}$ is a colocalizing subcategory of $\mathcal{B}(\mathfrak{C})$, and $\mathcal{F}(\mathfrak{C}) / \operatorname{Ker} \Phi_{\mathcal{B}(\mathfrak{C})} \approx \mathcal{B}(\mathfrak{C}), \mathcal{B}(\mathfrak{C}) / \operatorname{Ker} \Psi_{\mathfrak{C}} \approx \mathfrak{C}$, see $[\mathbf{3 9}]$. Since $\Psi_{\mathfrak{C}} \Phi_{\mathcal{B}(\mathfrak{C})} \mathbf{P}_{\mathcal{B}(\mathfrak{C})} \mathbf{Q}_{\mathfrak{C}}=\operatorname{Id}_{\mathfrak{C}}$ we have by $[\mathbf{7}]$ that $\mathcal{F}(\mathfrak{C}) / \operatorname{Ker} \Psi_{\mathfrak{C}} \Phi_{\mathcal{B}(\mathfrak{C})} \approx \mathfrak{C}$.

Finally we prove that the functor $\mathbf{P}_{\mathcal{B}(\mathfrak{C})} \mathbf{Q}_{\mathfrak{C}}$ realizes $\mathfrak{C}$ as the full subcategory $\mathcal{W}$ of projectiveinjective objects of $\mathcal{F}(\mathfrak{C})$. Clearly we have inclusions $\operatorname{Im}\left(\mathbf{P}_{\mathcal{B}(\mathfrak{C})} \mathbf{Q}_{\mathfrak{C}}\right) \subseteq \operatorname{Proj}(\mathcal{A B}(\mathfrak{C}))$ and $\operatorname{Im}\left(\mathbf{Q}_{\mathcal{A}(\mathfrak{C})} \mathbf{P}_{\mathfrak{C}}\right) \subseteq \operatorname{Inj}(\mathcal{B A}(\mathfrak{C}))$. Using the unique equivalence $D: \mathcal{A B}(\mathfrak{C}) \approx \mathcal{B} \mathcal{A}(\mathfrak{C})$ with the property $D \mathbf{P}_{\mathcal{B}(\mathfrak{C})} \mathbf{Q}_{\mathfrak{C}}=\mathbf{Q}_{\mathcal{A}(\mathfrak{C})} \mathbf{P}_{\mathfrak{C}}$, we deduce directly that $\operatorname{Im}\left(\mathbf{P}_{\mathcal{B}(\mathfrak{C})} \mathbf{Q}_{\mathfrak{C}}\right) \subseteq \operatorname{Proj}(\mathcal{A B}(\mathfrak{C})) \cap$ $\operatorname{Inj}(\mathcal{A B}(\mathfrak{C}))=\mathcal{W}$. Now if $X \in \mathcal{W}$, then $X$ is of the form $X=\mathbf{P}_{\mathcal{B}(\mathfrak{C})}(Y)$ for some $Y=$ $[A, f, B] \in \mathcal{B}(\mathfrak{C})$. Applying the kernel preserving functor $\mathbf{P}_{\mathcal{B}(\mathfrak{C})}$ to the exact sequence $0 \rightarrow$ $[A, f, B] \rightarrow \mathbf{Q}_{\mathfrak{C}}(A) \rightarrow \mathbf{Q}_{\mathfrak{C}}(B)$ in $\mathcal{B}(\mathfrak{C})$, we have the exact sequence $0 \rightarrow \mathbf{P}_{\mathcal{B}(\mathfrak{C})}[A, f, B] \rightarrow$ $\mathbf{P}_{\mathcal{B}(\mathfrak{C})} \mathbf{Q}_{\mathfrak{C}}(A) \rightarrow \mathbf{P}_{\mathcal{B}(\mathfrak{C})} \mathbf{Q}_{\mathfrak{C}}(B)$ in $\mathcal{F}(\mathfrak{C})$. Since $X=\mathbf{P}_{\mathcal{B}(\mathfrak{C})}[A, f, B]$ is injective, the morphism 
$\mathbf{P}_{\mathcal{B}(\mathfrak{C})}[A, f, B] \rightarrow \mathbf{P}_{\mathcal{B}(\mathfrak{C})} \mathbf{Q}_{\mathfrak{C}}(A)$ is split monic. Since $\mathbf{P}_{\mathcal{B}(\mathfrak{C})}$ is fully faithful, $[A, f, B] \rightarrow \mathbf{Q}_{\mathfrak{C}}(A)$ is also split monic. Hence $[A, f, B] \in \operatorname{Im}\left(\mathbf{Q}_{\mathfrak{C}}\right)$ and $X \in \operatorname{Im}\left(\mathbf{P}_{\mathcal{B}(\mathfrak{C})} \mathbf{Q}_{\mathfrak{C}}\right)$. We conclude that $\operatorname{Im}\left(\mathbf{P}_{\mathcal{B}(\mathfrak{C})} \mathbf{Q}_{\mathfrak{C}}\right)=\mathcal{W}$.

The following describes a relative version of the above Theorem.

Remark 6.2. Let $\mathfrak{C}$ be an exact category in the sense of Quillen $[40]$. Let $\mathbf{F}_{\mathfrak{C}}:=\mathbf{P}_{\mathcal{B}(\mathfrak{C})} \mathbf{Q}_{\mathfrak{C}}$ : $\mathfrak{C} \hookrightarrow \mathcal{F}(\mathfrak{C})$ be the full embedding of $\mathfrak{C}$ in its free abelian category and consider the full subcategory

$$
\begin{gathered}
\mathcal{L}:=\left\{F \in \mathcal{F}(\mathfrak{C}) \mid \text { there exists an exact sequence } \mathbf{F}_{\mathfrak{C}}(B) \stackrel{\mathbf{F}_{\mathfrak{C}}(f)}{\longrightarrow} \mathbf{F}_{\mathfrak{C}}(C) \rightarrow F \rightarrow 0\right. \\
\text { in } \mathcal{F}(\mathfrak{C}), \text { where } f: B \rightarrow C \text { is an admissible epic in } \mathfrak{C}\}
\end{gathered}
$$

Using the axioms of an exact category, it is not difficult to see that $\mathcal{L}$ is a Serre subcategory of $\mathcal{F}(\mathfrak{C})$. Consider the composite functor $\mathbf{E}: \mathfrak{C} \stackrel{\mathbf{F}_{\mathfrak{C}}}{\longrightarrow} \mathcal{F}(\mathfrak{C}) \stackrel{\pi}{\longrightarrow} \mathcal{F}(\mathfrak{C}) / \mathcal{L}$, where $\pi$ is the (exact) quotient functor. We leave to the reader to check that the functor $\mathbf{E}$ is fully faithful and embedds $\mathfrak{C}$ as an extension closed subcategory of $\mathcal{F}(\mathfrak{C}) / \mathcal{L}$. Moreover a sequence $A \rightarrow B \rightarrow C$ is an admissible short exact sequence in $\mathfrak{C}$ iff $0 \rightarrow \mathbf{E}(A) \rightarrow \mathbf{E}(B) \rightarrow \mathbf{E}(C) \rightarrow 0$ is a short exact sequence in $\mathcal{F}(\mathfrak{C}) / \mathcal{L}$. If $H: \mathfrak{C} \rightarrow \mathcal{H}$ is an exact functor to an abelian category $\mathcal{H}$, i.e. $H$ sends admissible short exact sequence in $\mathfrak{C}$ to short exact sequences in $\mathcal{H}$, then its unique exact extension $H^{\diamond}: \mathcal{F}(\mathfrak{C}) \rightarrow \mathcal{H}$ via $\mathbf{F}_{\mathfrak{C}}$, kills the objects of the Serre subcategory $\mathcal{L}$. Hence there exists a unique exact functor $\widehat{H}^{\diamond}: \mathcal{F}(\mathfrak{C}) / \mathcal{L} \rightarrow \mathcal{H}$ such that $\widehat{F}^{\diamond} \mathbf{E}=H$. It follows that the functor $\mathbf{E}: \mathfrak{C} \rightarrow \mathcal{F}(\mathfrak{C}) / \mathcal{L}$ is the universal exact functor out of $\mathfrak{C}$ to an abelian category. If $\mathfrak{C}$ carries the minimal exact structure, then $\mathcal{L}=0$ and we recover part 4 of Theorem 6.1.

We denote by $\mathcal{H}^{b}(\mathfrak{D})$ the bounded homotopy category of complexes over an additive category $\mathfrak{D}$, and by $\mathcal{D}^{b}(\mathfrak{D})$ the bounded derived category of an abelian category $\mathfrak{D}[\mathbf{5 0}]$. The following is a direct consequence of Theorem 6.1 .

Corollary 6.3. For any additive category $\mathfrak{C}$ there exists a triangle equivalence $\mathcal{H}^{b}(\mathcal{A}(\mathfrak{C})) \approx$ $\mathcal{H}^{b}(\mathcal{B}(\mathfrak{C}))$. If $\mathfrak{C}$ is coherent then the abelian categories $\mathcal{A}(\mathfrak{C}), \mathcal{B}(\mathfrak{C})$ are derived equivalent, i.e. there exists a triangle equivalence $\mathcal{D}^{b}(\mathcal{A}(\mathfrak{C})) \approx \mathcal{D}^{b}(\mathcal{B}(\mathfrak{C})$ ). In particular there exists an isomorphism: $\mathrm{K}_{0}(\mathcal{A}(\mathfrak{C})) \stackrel{\cong}{\longrightarrow} \mathrm{K}_{0}(\mathcal{B}(\mathfrak{C}))$.

Let Add be the category of additive categories and additive functors, and Abel be the category of abelian categories and exact functors. Copying the arguments of the proof of Theorem 3.4, we have the following result of $[\mathbf{1}]$.

Corollary 6.4. The forgetful functor $\mathcal{U}:$ Abel $\rightarrow$ Add is tripleable and admits as a left adjoint the functor $\mathcal{F}:=\mathcal{A B}:$ Add $\rightarrow$ Abel. Further $(\mathfrak{C}, F)$ is a $\mathcal{T}$-algebra for the triple $\mathcal{T}$ generated by $(\mathcal{F}, \mathcal{U})$ iff $\mathfrak{C}$ is an abelian category and $F=\Psi_{\mathfrak{C}} \Phi_{\mathcal{B}(\mathfrak{C})}$. Also $T:(\mathfrak{C}, F) \rightarrow(\mathfrak{D}, G)$ is a morphism of $\mathcal{T}$-algebras iff $T: \mathfrak{C} \rightarrow \mathfrak{D}$ is exact.

If Cat is the category of (small) categories and functors, then by the above Corollary and Remark 3.5, the forgetful functor $\mathcal{V}: \mathbf{A b e l} \rightarrow$ Cat has a left adjoint, which is obtained as the composition of the following five functors Cat $\stackrel{\mathbb{Z}}{\longrightarrow}$ PreAdd $\stackrel{\oplus}{\longrightarrow}$ Add $\stackrel{\perp}{\rightarrow} \operatorname{Idem} \stackrel{\mathcal{B}}{\longrightarrow} \operatorname{Ker} \stackrel{\mathcal{A}}{\longrightarrow}$ Abel, any one of which is a left adjoint of the corresponding forgetful functor (here PreAdd is the category of preadditive categories and additive functors).

If $\mathfrak{D}$ is abelian, we denote by $\operatorname{Ex}(\mathfrak{D}, \mathfrak{A} b)$ the category of exact functors from $\mathfrak{D}$ to the category of abelian groups. A direct consequence of Theorem 6.1 is the following.

Corollary 6.5. For any additive category $\mathfrak{C}$ there are equivalences:

$$
\operatorname{Mod}-\mathfrak{C} \approx \operatorname{Ex}\left(\mathcal{F}(\mathfrak{C})^{o p}, \mathfrak{A} b\right), \quad \mathfrak{C}-\operatorname{Mod} \approx \operatorname{Ex}(\mathcal{F}(\mathfrak{C}), \mathfrak{A} b)
$$


Let $\mathcal{H}$ be an abelian category with enough projectives and injectives. We say that $\mathcal{H}$ has dominant dimension $\geqslant n$, denoted by $\operatorname{dom} \cdot \operatorname{dim} \mathcal{H} \geqslant n$, if the first $n$-terms of an injective resolution of any projective object are projective.

Recognizing free abelian categories we have the following.

Theorem 6.6. For any category $\mathcal{F}$, the following are equivalent:

(1) $\mathcal{F}$ is a free abelian category.

(2) $\mathcal{F}^{o p}$ is a free abelian category.

(3) $\mathcal{F}$ is an abelian category with enough projectives and injectives, and moreover:

$$
\text { gl. } \operatorname{dim} \mathcal{F} \leqslant 2 \text { and } \operatorname{dom} \cdot \operatorname{dim} \mathcal{F} \geqslant 2 \text {. }
$$

If $\mathcal{F}$ is free abelian, then $\mathcal{F} \approx \mathcal{F}(\mathfrak{C})$, where $\mathfrak{C}$ is the full subcategory of projective-injective objects. Moreover either gl.dim $\mathcal{F}=0$ or gl.dim $\mathcal{F}=2=\operatorname{dom} . \operatorname{dim} \mathcal{F}$.

Proof. (1) $\Rightarrow(3)$ Suppose that $\mathcal{F}=\mathcal{F}(\mathfrak{C})=\mathcal{A B}(\mathfrak{C})$, for an additive category $\mathfrak{C}$. By Theorem 6.1 it suffices to prove that $\operatorname{dom} \cdot \operatorname{dim} \mathcal{F} \geqslant 2$. Let $[A, f, B] \in \mathcal{B}(\mathfrak{C})$. By Theorem 6.1(4) we have an exact sequence $0 \rightarrow \mathbf{P}_{\mathcal{B}(\mathfrak{C})}[A, f, B] \rightarrow \mathbf{P}_{\mathcal{B}(\mathfrak{C})} \mathbf{Q}_{\mathfrak{C}}(A) \rightarrow \mathbf{P}_{\mathcal{B}(\mathfrak{C})} \mathbf{Q}_{\mathfrak{C}}(B)$ in $\mathcal{F}(\mathfrak{C})$, in which $\mathbf{P}_{\mathcal{B}(\mathfrak{C})} \mathbf{Q}_{\mathfrak{C}}(A), \mathbf{P}_{\mathcal{B}(\mathfrak{C})} \mathbf{Q}_{\mathfrak{C}}(B)$ are projective-injective objects. Since any projective object of $\mathcal{F}$ is in the image of $\mathbf{P}_{\mathcal{B}(\mathfrak{C})}$, we have $\operatorname{dom} \cdot \operatorname{dim} \mathcal{F} \geqslant 2$.

$(3) \Rightarrow(1)$ Let $\mathfrak{C}$ be the full subcategory $\operatorname{Proj}(\mathcal{F}) \cap \operatorname{Inj}(\mathcal{F})$ of projective-injectives in $\mathcal{F}$, and let $\mathcal{J}: \mathfrak{C} \hookrightarrow \mathcal{F}$ be the inclusion. Consider the unique exact functor $\mathcal{J}^{\diamond}: \mathcal{F}(\mathfrak{C}) \rightarrow$ $\mathcal{F}$, with $\mathcal{J}^{\diamond}\left(\mathbf{P}_{\mathcal{B}(\mathfrak{C})} \mathbf{Q}_{\mathfrak{C}}\right)=\mathcal{J}$. We recall from the proof of Theorem 6.1, that $\mathcal{J}^{\diamond}=\left(\mathcal{J}^{*}\right)^{!}$, where $\mathcal{J}^{*}: \mathcal{B}(\mathfrak{C}) \rightarrow \mathcal{F}$ is the unique kernel preserving functor, with $\mathcal{J}^{*} \mathbf{Q}_{\mathfrak{C}}=\mathcal{J}$, and $\mathcal{J}^{\diamond}=$ $\left(\mathcal{J}^{*}\right)^{!}: \mathcal{F}(\mathfrak{C}) \rightarrow \mathcal{F}$ is the unique cokernel preserving functor, with $\mathcal{J}^{\diamond} \mathbf{P}_{\mathcal{B}(\mathfrak{C})}=\mathcal{J}^{*}$. Since by construction $\mathcal{J}^{*}[A, f, B]=\operatorname{Ker}(f)$, and since $\mathfrak{C} \subseteq \operatorname{Inj}(\mathcal{F})$, it is trivial to see that $\mathcal{J}^{*}$ is fully faithful, and $\operatorname{Im}\left(\mathcal{J}^{*}\right)$ consists of all objects $X \in \mathcal{F}$ having a copresentation $0 \rightarrow X \rightarrow A \rightarrow B$, with $A, B \in \mathfrak{C}$. Since gl.dim $\mathcal{F} \leqslant 2$, we have $\operatorname{Im}\left(\mathcal{J}^{*}\right) \subseteq \operatorname{Proj}(\mathcal{F})$. Since $\operatorname{dom}$.dim $\mathcal{F} \geqslant 2$, we have $\operatorname{Proj}(\mathcal{F}) \subseteq \operatorname{Im}\left(\mathcal{J}^{*}\right)$. Hence $\operatorname{Proj}(\mathcal{F})=\operatorname{Im}\left(\mathcal{J}^{*}\right)$. Identifying $\mathcal{B}(\mathfrak{C})=\operatorname{Im}\left(\mathcal{J}^{*}\right)$, Corollary 3.10 shows that $\mathcal{J}^{\diamond}$ is an equivalence.

If $\mathcal{F}$ is not semisimple, then by Theorem 6.1 , gl.dim $\mathcal{F}=2$. If $\operatorname{dom} \cdot \operatorname{dim} \mathcal{F} \geqslant 3$, then the last term of an injective resolution of any projective object of $\mathcal{F}$ is projective-injective. Hence the injective resolution splits and any projective object of $\mathcal{F}$ is injective. This implies trivially that gl.dim $\mathcal{F}=0$ and this is not the case. Hence gl.dim $\mathcal{F}=2=\operatorname{dom} . \operatorname{dim} \mathcal{F}$, if $\mathcal{F}$ is not semisimple.

$(1) \Leftrightarrow(2)$ Follows from the equivalence $\mathcal{F}\left(\mathfrak{C}^{o p}\right) \approx \mathcal{F}(\mathfrak{C})^{o p}$.

¿From now on we rename free abelian categories and we call them Auslander categories. This terminology is justified by the fact that an Artin algebra $\Lambda$ is called an Auslander algebra iff $\operatorname{gl} \cdot \operatorname{dim} \Lambda \leqslant 2$ and $\operatorname{dom} \cdot \operatorname{dim} \Lambda \geqslant 2$, i.e. iff $\bmod -\Lambda$ is an Auslander category [8]. Thus we see that Auslander categories are universal objects: they are precisely the free abelian categories, and there are enough of them: any additive category is embedded in its Auslander category.

Example 6.7. If $\Lambda$ is a ring, then $\mathcal{F}\left(\mathcal{P}_{\Lambda^{o p}}\right)^{o p} \approx \mathcal{F}\left(\left(\mathcal{P}_{\Lambda^{o p}}\right)^{o p}\right) \approx \mathcal{F}\left(\mathcal{P}_{\Lambda}\right)$. Hence there exist a duality between the Auslander categories of the categories of finitely generated projective left and right modules. But $\mathcal{F}\left(\mathcal{P}_{\Lambda}\right)^{o p} \approx(\bmod -\Lambda)-\bmod$ and $\mathcal{F}\left(\mathcal{P}_{\Lambda}\right) \approx(\Lambda-\bmod )-\bmod$. So there exists a duality between the Auslander categories $(\Lambda-\bmod )-\bmod ,(\bmod -\Lambda)-\bmod$. The existence of this last duality is a result of [27]; its explicit description is left to the reader.

Remark 6.8. (1) Obviously $\mathcal{A}(\mathfrak{C})$ is an Auslander category iff $\mathfrak{C} \cong \mathcal{B}(\mathfrak{D})$ for an additive category $\mathfrak{D}$. Dually $\mathcal{B}(\mathfrak{C})$ is an Auslander category iff $\mathfrak{C} \cong \mathcal{A}(\mathfrak{D})$ for an additive category $\mathfrak{D}$. If $\mathfrak{C}$ is abelian with enough projectives and injectives, then $\mathcal{A}(\operatorname{Proj}(\mathfrak{C})) \approx \mathfrak{C} \approx \mathcal{B}(\operatorname{Inj}(\mathfrak{C}))$. Hence $\mathcal{A}(\mathfrak{C})$ is the Auslander category of $\operatorname{Inj}(\mathfrak{C})$ and $\mathcal{B}(\mathfrak{C})$ is the Auslander category of Proj( $\mathfrak{C})$. If $\mathfrak{C}$ is Frobenius then by Theorem 6.1 we deduce that $\mathcal{A}(\mathfrak{C}) \approx \mathcal{B}(\mathfrak{C})$, so $\mathfrak{C}$ is dualizing. 
(2) If $\mathfrak{C}$ is dualizing, then $\mathcal{A}(\mathfrak{C}), \mathcal{B}(\mathfrak{C}), \mathcal{F}(\mathfrak{C}), \mathcal{A}\left(\mathfrak{C}^{\circ p}\right), \mathcal{B}\left(\mathfrak{C}^{o p}\right), \mathcal{F}\left(\mathfrak{C}^{\circ p}\right)$ and also their induced stable categories modulo projectives or injectives are dualizing.

(3) It is easy to see that if $\mathfrak{C}$ is $\mathrm{AB} 4$ or $\mathrm{AB} 4^{*}$, then so is $\mathcal{F}(\mathfrak{C})$. However if $\mathfrak{C}$ is $\mathrm{AB} 5$, then $\mathcal{F}(\mathfrak{C})$ is AB5 only if $\mathfrak{C}$ is spectral, i.e. if any short exact sequence in $\mathfrak{C}$ splits.

If $X$ is an object in an additive category $\mathfrak{C}$, then we define the Auslander category $\mathcal{F}(X)$ over $X$ to be the Auslander category $\mathcal{F}(\operatorname{add}(X))$, where $\operatorname{add}(X)$ is the full subcategory of $\mathfrak{C}$ consisting of all direct summands of finite direct sums of copies of $X$. An arbitrary additive category $\mathfrak{C}$ is called representation finite iff $\mathfrak{C}$ has a representation generator, i.e. an object $X$ such that $\mathfrak{C}=\operatorname{add}(X)$. Consider now the following classes of abelian categories:

$\mathcal{S}$ is the class of equivalence classes of (representation-finite) abelian categories $\mathfrak{C}$ with enough injectives, such that $\operatorname{Inj}(\mathfrak{C})$ is right coherent.

$\mathcal{R}$ is the class of equivalence classes of (representation-finite) abelian categories $\mathfrak{C}$ with enough projectives, such that $\operatorname{Proj}(\mathfrak{C})$ is left coherent.

$\mathcal{T}$ is the class of equivalence classes of Auslander categories $\mathcal{F}$, such that $\operatorname{Inj}(\mathcal{F}) \cap \operatorname{Proj}(\mathcal{F})$ is coherent (and $\operatorname{Proj}(\mathcal{F})$ is representation finite).

The next result generalizes the well-known correspondence between Morita equivalence classes of representation-finite Artin rings and Auslander Artin rings, see [2], [3].

Corollary 6.9. There are bijections

$$
\chi: \mathcal{S} \rightarrow \mathcal{T} \quad \text { and } \quad \rho: \mathcal{R} \rightarrow \mathcal{T}
$$

defined by: $\chi(\mathfrak{C})=\mathcal{A}(\mathfrak{C}), \quad \rho(\mathfrak{D})=\mathcal{B}(\mathfrak{D})$.

Proof. We use throughout that $\mathfrak{D}=\operatorname{Inj}(\mathcal{F}(\mathfrak{D})) \cap \operatorname{Proj}(\mathcal{F}(\mathfrak{D}))$, if $\mathcal{F}=\mathcal{F}(\mathfrak{D})$. Suppose that $\mathfrak{C} \in \mathcal{S}$. Since $\mathfrak{C}$ has enough injectives, by Corollary 3.10 we have $\mathfrak{C} \approx \mathcal{B}(\operatorname{Inj}(\mathfrak{C}))$. Hence $\chi(\mathfrak{C})=\mathcal{A}(\mathfrak{C}) \approx \mathcal{A}(\mathcal{B}(\operatorname{Inj}(\mathfrak{C})))=\mathcal{F}(\operatorname{Inj}(\mathfrak{C}))$ is free. $\operatorname{Since} \operatorname{Inj}(\mathcal{F}) \cap \operatorname{Proj}(\mathcal{F}) \approx \operatorname{Inj}(\mathfrak{C})$, and since $\operatorname{Inj}(\mathfrak{C})$ is always left coherent (because $\mathfrak{C}$ has enough injectives), we have that $\operatorname{Inj}(\mathcal{F}(\operatorname{Inj}(\mathfrak{C}))) \cap$ $\operatorname{Proj}(\mathcal{F}(\operatorname{Inj}(\mathfrak{C})))$ is coherent. If $\mathfrak{C}$ is representation finite, then $\operatorname{Proj}(\mathcal{F}(\operatorname{Inj}(\mathfrak{C}))=\mathcal{B}(\operatorname{Inj}(\mathfrak{C}))=\mathfrak{C}$ is representation finite. Hence $\chi(\mathfrak{C}) \in \mathcal{T}$. If $\mathcal{F}=\mathcal{F}(\mathfrak{D}) \in \mathcal{T}$, then define $\psi: \mathcal{T} \rightarrow \mathcal{S}$ by $\psi(\mathcal{F})=\mathcal{B}(\operatorname{Inj}(\mathcal{F}) \cap \operatorname{Proj}(\mathcal{F}))$. Suppose that $\mathcal{F}=\mathcal{F}(\mathfrak{D})$. Since $\operatorname{Inj}(\mathcal{F}) \cap \operatorname{Proj}(\mathcal{F}) \approx \mathfrak{D}$ is coherent, by Proposition $4.5, \mathcal{B}(\mathfrak{D})$ is abelian, and $\operatorname{Inj}(\mathcal{B}(\mathfrak{D})) \approx \mathfrak{D}$ is right coherent. If $\operatorname{Proj}(\mathcal{F})$ is representation finite, then $\psi(\mathcal{F})=\mathcal{B}(\mathfrak{D})=\operatorname{Proj}(\mathcal{F})$ is representation finite. Hence $\psi(\mathcal{F}) \in \mathcal{S}$. If $\mathfrak{C} \in \mathcal{S}$, then $\psi \chi(\mathfrak{C})=\psi[\mathcal{A}(\mathfrak{C})]=\psi[\mathcal{A}(\mathcal{B}(\operatorname{Inj}(\mathfrak{C})))]=\psi[\mathcal{F}(\operatorname{Inj}(\mathfrak{C}))]=$ $\mathcal{B}[\operatorname{Inj}(\mathcal{F}(\operatorname{Inj}(\mathfrak{C}))) \cap \operatorname{Proj}(\mathcal{F}(\operatorname{Inj}(\mathfrak{C})))]=\mathcal{B}(\operatorname{Inj}(\mathfrak{C}))=\mathfrak{C}$. If $\mathcal{F}=\mathcal{F}(\mathfrak{D}) \in \mathcal{T}$, then $\chi \psi(\mathcal{F})=$ $\chi[\mathcal{B}(\operatorname{Inj}(\mathcal{F}) \cap \operatorname{Proj}(\mathcal{F}))]=\chi[\mathcal{B}(\mathfrak{D})]=\mathcal{A B}(\mathfrak{D})=\mathcal{F}(\mathfrak{D})=\mathcal{F}$. We deduce that $\chi$ is the inverse of $\psi$. The other part follows similarly.

We close this section by introducing a new dimension for an abelian category. From now on $\mathfrak{C}$ will denote an abelian category. Let $\mathbf{F}_{\mathfrak{C}}:=\mathbf{P}_{\mathcal{B}(\mathfrak{C})} \mathbf{Q}_{\mathfrak{C}}: \mathfrak{C} \rightarrow \mathcal{F}(\mathfrak{C})$ be the full embedding realizing $\mathfrak{C}$ as the full subcategory of projective-injective objects in its free abelian category. Then the functor $\mathbf{Q}_{\mathfrak{C}}$ admits an exact right adjoint $\Psi_{\mathfrak{C}}: \mathcal{B}(\mathfrak{C}) \rightarrow \mathfrak{C}$ with kernel $\mathcal{B}^{m}(\mathfrak{C})$, and the functor $\mathbf{P}_{\mathcal{B}(\mathfrak{C})}: \mathcal{A B}(\mathfrak{C}) \rightarrow \mathcal{B}(\mathfrak{C})$ admits a left adjoint $\Phi_{\mathcal{B}(\mathfrak{C})}: \mathcal{A B}(\mathfrak{C}) \rightarrow \mathfrak{C}$ with kernel $\mathcal{A}_{e}(\mathcal{B}(\mathfrak{C}))$. From now on we set $\Omega(\mathfrak{C}):=\mathcal{A}_{e}(\mathcal{B}(\mathfrak{C})) \approx \mathcal{B}^{m}(\mathcal{A}(\mathfrak{C}))$ and $\mathbf{R}_{\mathfrak{C}}:=\Psi_{\mathfrak{C}} \Phi_{\mathcal{B}(\mathfrak{C})}$, so that we have a short exact sequence of abelian categories

$$
0 \longrightarrow \Omega(\mathfrak{C}) \longrightarrow \mathcal{F}(\mathfrak{C}) \stackrel{\mathbf{R}_{\mathfrak{C}}}{\longrightarrow} \mathfrak{C} \longrightarrow 0
$$

which we call a free presentation of the abelian category $\mathfrak{C}$. Since $\Omega(\mathfrak{C})$ is again an abelian category the above procedure can be continued. In this way we obtain free presentations:

$$
0 \longrightarrow \Omega^{n+1}(\mathfrak{C}) \longrightarrow \mathcal{F}^{n}(\mathfrak{C}) \stackrel{\mathbf{R}_{\Omega^{n}(\mathfrak{C})}}{\longrightarrow} \Omega^{n}(\mathfrak{C}) \longrightarrow 0
$$


for any $n \geqslant 0$, where $\Omega^{0}(\mathfrak{C}):=\mathfrak{C}$ and $\mathcal{F}^{0}(\mathfrak{C})=\mathcal{F}(\mathfrak{C})$. Splicing the above exact sequences of abelian categories we obtain the following diagram

$$
\cdots \longrightarrow \mathcal{F}^{n}(\mathfrak{C}) \stackrel{\mathbf{F}_{\mathfrak{C}}^{n}}{\longrightarrow} \mathcal{F}^{n-1}(\mathfrak{C}) \longrightarrow \cdots \longrightarrow \mathcal{F}^{1}(\mathfrak{C}) \stackrel{\mathbf{F}_{\mathfrak{C}}^{1}}{\longrightarrow} \mathcal{F}^{0}(\mathfrak{C}) \stackrel{\mathbf{F}_{\mathfrak{C}}^{0}}{\longrightarrow} \mathfrak{C} \longrightarrow 0
$$

where each $\mathcal{F}^{n}(\mathfrak{C})$ is free abelian and each $\mathbf{F}^{n}: \mathcal{F}^{n}(\mathfrak{C}) \rightarrow \mathcal{F}^{n-1}(\mathfrak{C})$ is an exact functor with image the abelian category $\Omega^{n}(\mathfrak{C})$. We call the above diagram a free resolution of the abelian category $\mathfrak{C}$. The above considerations suggest that the following concept should be useful.

Definition 6.10. The Auslander dimension $\operatorname{Dim}_{\mathbf{A}} C$ of an abelian category $\mathfrak{C}$ is the smallest integer $n$ such that $\Omega^{n}(\mathfrak{C})$ is free abelian. If no such integer exists, then we set $\operatorname{Dim}_{\mathbf{A}} \mathfrak{C}=\infty$.

Observe that by construction we have the folowing exact commutative diagram of abelian categories and exact functors:

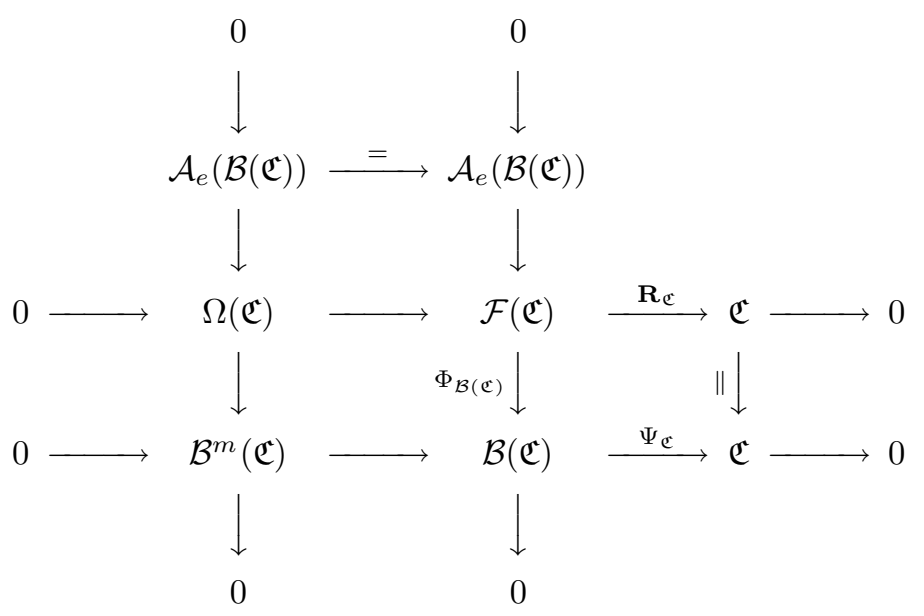

Observe that if $\mathfrak{C}$ has enough projectives and enough injectives, then $\mathcal{A}_{e}(\mathcal{B}(\mathfrak{C})) \approx \mathcal{A}(\underline{\mathcal{B}(\mathfrak{C})})$ and $\mathcal{B}^{m}(\mathfrak{C}) \approx \mathcal{B}(\overline{\mathfrak{C}})$. Hence the left column is isomorphic to

$$
0 \longrightarrow \mathcal{A}(\underline{\mathcal{B}(\mathfrak{C})}) \longrightarrow \Omega(\mathfrak{C}) \longrightarrow \mathcal{B}(\overline{\mathfrak{C}}) \longrightarrow 0
$$

It is easy to see that $\operatorname{Dim}_{\mathbf{A}} \mathfrak{C}=0$ iff gl.dimC $=0$. We refer to $[\mathbf{1 4}]$ for a discussion of the Auslander dimension of an abelian category $\mathfrak{C}$ and its connection with the Krull-Gabriel dimension of $\mathfrak{C}$ and the representation dimension in the sense of $[\mathbf{2}]$.

\section{Modules over the Freyd Categories}

Throughout this section we assume that $\mathfrak{C}$ is a skeletally small additive category.

\subsection{Flat and FP-Injective Functors}

Let $\mathcal{W} \mathcal{K}\left(\mathfrak{C}^{o p}, \mathfrak{A} b\right)$, resp. $\mathcal{W C}\left(\mathfrak{C}^{o p}, \mathfrak{A} b\right)$, be the full subcategory of Mod-C consisting of all functors sending weak-kernel, resp. weak-cokernel, sequences to exact sequences. The categories $\mathcal{W K}(\mathfrak{C}, \mathfrak{A} b), \mathcal{W C}(\mathfrak{C}, \mathfrak{A} b)$ are defined dually. We recall [46] that a right $\mathfrak{C}$-module $F$ is called $F P$-injective iff $\mathcal{E} x t^{1}(G, F)=0, \forall G \in \bmod -\mathfrak{C}$. We denote by FPInj(Mod-C $)$, resp. Flat(Mod-C $)$, the full subcategory of FP-injective, resp. flat functors [37].

Proposition 7.1. (1) If $\mathfrak{C}$ is right coherent, there are equivalences:

$$
\begin{gathered}
\operatorname{Ex}(\mathcal{A}(\mathfrak{C}), \mathfrak{A} b) \approx \mathcal{W} \mathcal{K}(\mathfrak{C}, \mathfrak{A} b)=\operatorname{Flat}(\mathfrak{C}-\operatorname{Mod}) \\
\operatorname{Ex}\left(\mathcal{A}(\mathfrak{C})^{o p}, \mathfrak{A} b\right) \approx \mathcal{W} \mathcal{K}\left(\mathfrak{C}^{o p}, \mathfrak{A} b\right)=\operatorname{FPInj}(\operatorname{Mod}-\mathfrak{C}) .
\end{gathered}
$$


(2) If $\mathfrak{C}$ is left coherent, there are equivalences:

$$
\begin{gathered}
\operatorname{Ex}\left(\mathcal{B}(\mathfrak{C})^{o p}, \mathfrak{A} b\right) \approx \mathcal{W C}\left(\mathfrak{C}^{o p}, \mathfrak{A} b\right)=\operatorname{Flat}(\operatorname{Mod}-\mathfrak{C}) \\
\operatorname{Ex}(\mathcal{B}(\mathfrak{C}), \mathfrak{A} b) \approx \mathcal{W C}(\mathfrak{C}, \mathfrak{A} b)=\operatorname{FPInj}(\mathfrak{C}-\operatorname{Mod}) .
\end{gathered}
$$

Proof. (1) The first equivalences are consequences of Corollary 4.7. Let $F$ be in Mod-C $\mathfrak{C}$, and let $G=\operatorname{Coker}(-, f)$ be in mod-C $\mathfrak{C}$, where $f: B \rightarrow C$ is a morphism in $\mathfrak{C}$. Consider the weak kernel sequence $K_{f}^{\bullet}$ over $f$ as in section 4 . Then $G$ has a projective resolution $\cdots \rightarrow \mathfrak{C}\left(-, K_{f}^{1}\right) \rightarrow$ $\mathfrak{C}\left(-, K_{f}^{0}\right) \rightarrow \mathfrak{C}(-, B) \rightarrow \mathfrak{C}(-, C) \rightarrow G \rightarrow 0$. By Yoneda's Lemma, $\mathcal{E} x t_{\text {Mod }-\mathfrak{C}}^{i}(G, F)=$ the cohomology of the complex $0 \rightarrow F(C) \rightarrow F(B) \rightarrow F\left(K_{f}^{0}\right) \rightarrow F\left(K_{f}^{1}\right) \rightarrow \cdots$. Hence $F$ is an

FP-injective object iff $F$ is in $\mathcal{W} \mathcal{K}\left(\mathfrak{C}^{o p}\right)$. Let now $F$ be in $\mathfrak{C}$-Mod and let $A \stackrel{g}{\rightarrow} B \stackrel{f}{\rightarrow} C$ be a diagram in $\mathfrak{C}$ with $g$ a weak kernel of $f$. This diagram can be extended to a weak kernel sequence $K_{f}^{\bullet}$ since $\mathfrak{C}$ is right coherent. If $G=\operatorname{Coker}(-, f)$, then $G$ has a projective resolution $\cdots \rightarrow \mathfrak{C}(-, A) \rightarrow \mathfrak{C}(-, B) \rightarrow \mathfrak{C}(-, C) \rightarrow G \rightarrow 0$. Then $\mathcal{T}_{\text {or }}{ }_{*}^{\mathfrak{C}}(G, F)=$ the homology of the complex $\cdots \rightarrow F(A) \rightarrow F(B) \rightarrow F(C) \rightarrow 0$. Since $F$ is flat iff $\mathcal{T}_{\text {or }}^{\mathfrak{C}}(G, F)=0, \forall n \geqslant 1$, $\forall G \in \bmod -\mathfrak{C}$, the claim follows. Part $(2)$ is similar.

Consider now the full imbeddings $\mathbf{P}: \mathfrak{C} \rightarrow \mathcal{A}(\mathfrak{C}), \mathbf{Q}: \mathfrak{C} \rightarrow \mathcal{B}(\mathfrak{C})$ of $\mathfrak{C}$ in its Freyd categories. The functors $\mathbf{P}, \mathbf{Q}$ induce exact restriction functors

$$
\begin{aligned}
& \widehat{\mathbf{P}}: \operatorname{Mod}-\mathcal{A}(\mathfrak{C}) \rightarrow \operatorname{Mod}-\mathfrak{C}, \quad \widetilde{\mathbf{P}}: \mathcal{A}(\mathfrak{C})-\operatorname{Mod} \rightarrow \mathfrak{C}-\operatorname{Mod} \\
& \widehat{\mathbf{Q}}: \operatorname{Mod}-\mathcal{B}(\mathfrak{C}) \rightarrow \operatorname{Mod}-\mathfrak{C}, \quad \widetilde{\mathbf{Q}}: \mathcal{B}(\mathfrak{C})-\operatorname{Mod} \rightarrow \mathfrak{C}-\operatorname{Mod}
\end{aligned}
$$

defined in the obvious way: $\widehat{\mathbf{P}}(F)=F \mathbf{P}$, and so on. Now consider the functors

$$
\begin{gathered}
\widehat{\mathrm{R}}: \operatorname{Mod}-\mathfrak{C} \rightarrow \operatorname{Mod}-\mathcal{A}(\mathfrak{C}), \quad \widetilde{\mathrm{R}}: \mathfrak{C}-\operatorname{Mod} \rightarrow \mathcal{A}(\mathfrak{C})-\operatorname{Mod} \\
\widehat{\mathrm{L}}: \operatorname{Mod}-\mathfrak{C} \rightarrow \operatorname{Mod}-\mathcal{B}(\mathfrak{C}), \quad \widetilde{\mathrm{L}}: \mathfrak{C}-\operatorname{Mod} \rightarrow \mathcal{B}(\mathfrak{C})-\operatorname{Mod}
\end{gathered}
$$

defined using the universal properties of $\mathcal{A}(\mathfrak{C}), \mathcal{B}(\mathfrak{C})$ as follows: $\widehat{\mathrm{R}}(F)=F^{!}, \widehat{\mathrm{L}}(F)=F^{*}$ and similarly for $\widetilde{\mathrm{R}}, \widetilde{\mathrm{L}}$. Clearly we have adjoint pairs $(\widehat{\mathbf{P}}, \widehat{\mathrm{R}}),(\widehat{\mathrm{L}}, \widehat{\mathbf{Q}}),(\widetilde{\mathbf{Q}}, \widetilde{\mathrm{L}}),(\widetilde{\mathrm{R}}, \widetilde{\mathbf{P}})$ and the functors $\widehat{R}, \widehat{L}, \widetilde{R}, \widetilde{L}$ are fully faithful, since obviously:

$$
\widehat{\mathbf{P}} \widehat{\mathrm{R}}=\operatorname{Id}_{\text {Mod-c }}, \quad \widehat{\mathbf{Q}} \widehat{\mathrm{L}}=\operatorname{Id}_{\text {Mod }-\mathfrak{C}}, \quad \widetilde{\mathbf{P}} \widetilde{\mathrm{R}}=\operatorname{Id}_{\mathfrak{C}-\operatorname{Mod}}, \quad \widetilde{\mathbf{Q}} \widetilde{\mathrm{L}}=\mathrm{Id}_{\mathfrak{C}-\text { Mod }}
$$

It is easy to see that $\operatorname{Ker}(\widehat{\mathbf{P}})=\operatorname{Mod}-\underline{\mathcal{A}(\mathfrak{C})}, \operatorname{Ker}(\widetilde{\mathbf{Q}})=\overline{\mathcal{B}(\mathfrak{C})}-\operatorname{Mod}$ which are localizing subcategories and $\operatorname{Ker}(\widetilde{\mathbf{P}})=\mathcal{A}(\mathfrak{C})-\operatorname{Mod}, \operatorname{Ker}(\widehat{\mathbf{Q}})=\operatorname{Mod}-\overline{\mathcal{B}(\mathfrak{C})}$ which are colocalizing subcategories. In particular we have the following exact sequences of Grothendieck categories:

$$
\begin{gathered}
\operatorname{Mod}-\underline{\mathcal{A}(\mathfrak{C})} \hookrightarrow \operatorname{Mod}-\mathcal{A}(\mathfrak{C}) \underset{\widehat{\mathrm{R}}}{\stackrel{\widehat{\mathbf{P}}}{\rightleftarrows}} \operatorname{Mod}-\mathfrak{C}, \quad \overline{\mathcal{B}(\mathfrak{C})}-\operatorname{Mod} \hookrightarrow \mathcal{B}(\mathfrak{C})-\operatorname{Mod} \underset{\widetilde{\mathrm{L}}}{\underset{\mathrm{Q}}{\rightleftarrows}} \mathfrak{C}-\operatorname{Mod}, \\
\operatorname{Mod}-\overline{\mathcal{B}(\mathfrak{C})} \hookrightarrow \operatorname{Mod}-\mathcal{B}(\mathfrak{C}) \underset{\widehat{\mathrm{L}}}{\stackrel{\widehat{\mathbf{Q}}}{\rightleftarrows}} \operatorname{Mod}-\mathfrak{C}, \quad \underline{\mathcal{A}(\mathfrak{C})}-\operatorname{Mod} \hookrightarrow \mathcal{A}(\mathfrak{C})-\operatorname{Mod} \underset{\widetilde{\mathrm{R}}}{\stackrel{\widetilde{\mathbf{P}}}{\rightleftarrows}} \mathfrak{C}-\operatorname{Mod} .
\end{gathered}
$$

Corollary 7.2. (1) There are equivalences

$$
\begin{gathered}
\operatorname{Im}(\widetilde{\mathrm{L}}) \approx \mathfrak{C}-\operatorname{Mod} \approx \operatorname{Lex}(\mathcal{B}(\mathfrak{C}), \mathfrak{A} b)=\operatorname{Flat}(\mathcal{B}(\mathfrak{C})-\operatorname{Mod}) \approx \operatorname{Ex}(\mathcal{F}(\mathfrak{C}), \mathfrak{A} b), \\
\operatorname{Im}(\widetilde{\mathrm{R}}) \approx \mathfrak{C}-\operatorname{Mod} \approx \operatorname{Rex}(\mathcal{A}(\mathfrak{C}), \mathfrak{A} b)=\operatorname{FPInj}(\mathcal{A}(\mathfrak{C})-\operatorname{Mod}) \approx \operatorname{Ex}(\mathcal{F}(\mathfrak{C}), \mathfrak{A} b), \\
\operatorname{Im}(\widehat{\mathrm{R}}) \approx \operatorname{Mod}-\mathfrak{C} \approx \operatorname{Lex}\left(\mathcal{A}(\mathfrak{C})^{\mathrm{op}}, \mathfrak{A} b\right)=\operatorname{Flat}(\operatorname{Mod}-\mathcal{A}(\mathfrak{C})) \approx \operatorname{Ex}\left(\mathcal{F}(\mathfrak{C})^{\mathrm{op}}, \mathfrak{A} b\right), \\
\operatorname{Im}(\widehat{\mathrm{L}}) \approx \operatorname{Mod}-\mathfrak{C} \approx \operatorname{Rex}\left(\mathcal{B}(\mathfrak{C})^{\mathrm{op}}, \mathfrak{A} b\right)=\operatorname{FPInj}(\operatorname{Mod}-\mathcal{B}(\mathfrak{C})) \approx \operatorname{Ex}\left(\mathcal{F}(\mathfrak{C})^{\mathrm{op}}, \mathfrak{A} b\right)
\end{gathered}
$$


(2) If $\mathfrak{C}$ is right coherent there are equivalences

$$
\operatorname{FPInj}(\operatorname{Mod}-\mathcal{A}(\mathfrak{C})) \approx \operatorname{Ex}\left(\mathcal{A} \mathcal{A}(\mathfrak{C})^{\mathrm{op}}, \mathfrak{A} b\right), \operatorname{Flat}(\mathcal{A}(\mathfrak{C})-\operatorname{Mod}) \approx \operatorname{Ex}(\mathcal{A} \mathcal{A}(\mathfrak{C}), \mathfrak{A} b) .
$$

(3) If $\mathfrak{C}$ is left coherent there are equivalences

$$
\operatorname{Flat}(\operatorname{Mod}-\mathcal{B}(\mathfrak{C})) \approx \operatorname{Ex}\left(\mathcal{B B}(\mathfrak{C})^{\mathrm{op}}, \mathfrak{A} b\right), \operatorname{FPInj}(\mathcal{B}(\mathfrak{C})-\operatorname{Mod}) \approx \operatorname{Ex}(\mathcal{B B}(\mathfrak{C}), \mathfrak{A} b) .
$$

Proof. (1) The first equivalences hold since the functors $\widehat{R}, \widehat{L}, \widetilde{R}, \widetilde{L}$ are fully faithful. The second are true because of Corollary 3.9. The remaining equivalences of (1) as well as of (2) follow from Proposition 7.1, if we replace $\mathfrak{C}$ by its Freyd categories.

Remark 7.3. (1) There are equivalences:

$$
\begin{aligned}
\bmod -\mathcal{B}(\mathfrak{C}) & \approx \mathcal{F}(\mathfrak{C}), \quad \mathcal{A}(\mathfrak{C})-\bmod \approx \mathcal{F}(\mathfrak{C})^{o p} \approx \mathcal{F}\left(\mathfrak{C}^{o p}\right), \\
\bmod -\mathcal{A}(\mathfrak{C}) & \approx \mathcal{A}(\mathcal{A}(\mathfrak{C})), \quad \mathcal{B}(\mathfrak{C})-\bmod \approx \mathcal{B}(\mathcal{B}(\mathfrak{C}))^{o p} \approx \mathcal{A}\left(\mathcal{A}\left(\mathfrak{C}^{o p}\right)\right) .
\end{aligned}
$$

(2) For an additive category $\mathfrak{C}$, let $\prod(\mathfrak{C}-\operatorname{Mod}, \mathfrak{A} b)$ be the category of functors commuting with direct limits and products. Let $\vec{F}(\mathfrak{C})$ be the Auslander category of $\mathfrak{C}$. If $\mathfrak{C}$ has weak cokernels, then it follows from $[\mathbf{3 1}]$ that we have an equivalence

$$
\mathcal{F}(\mathfrak{C}) \stackrel{\approx}{\longrightarrow} \underline{\prod}(\mathfrak{C}-\operatorname{Mod}, \mathfrak{A} b) .
$$

(3) We observe that the Freyd categories are such that the flat, FP-injective modules over them are abelian and reflective, coreflective respectively in their whole module categories. The results of the next subsection generalize these observations.

\subsection{Definable and Homologically Finite Subcategories}

We recall $[\mathbf{4 3}]$ that a short exact sequence in the functor category Mod-C is called pure if any finite presented functor is a relative projective for it. A monic in a pure exact sequence is called a pure monic. Let $\mathcal{H} \subseteq \operatorname{Mod}-\mathfrak{C}$ be a full subcategory. Following [32], $\mathcal{H}$ is called definable if $\mathcal{H}$ is closed under direct limits, products and pure subobjects. In the following we set $\mathfrak{\aleph}(\mathfrak{C})$ to be the cardinality of the set Iso( $(\mathfrak{C})$ of isomorphism classes of objects of $\mathfrak{C}$. Also if $M \in \operatorname{Mod}-\mathfrak{C}$, we denote by $|M|$ the cardinality of $\bigcup_{X \in \operatorname{Iso}(\mathfrak{C})} M(X)$.

Lemma 7.4. Let $N \hookrightarrow M$ be an inclusion in Mod-C . Then there exists a pure subobject $T \hookrightarrow M$ such that: $N \hookrightarrow T \hookrightarrow M$ and $|T| \leqslant \max \left\{\aleph_{0}, \aleph(\mathfrak{C}),|M|\right\}$.

Proof. In [34] it is proved that there exists a subobject $T$ of $M$ which contains $N$ and satisfies $|T| \leqslant \max \left\{\aleph_{0}, \aleph(\mathfrak{C}),|M|\right\}$. Following the inductive construction of $T$ in [34], it is not difficult to see that we can choose $T$ to be a pure subobject of $M$.

Proposition 7.5. Let $\mathcal{H}$ be a full additive subcategory of Mod-C , closed under direct limits and pure subobjects. Then $\mathcal{H}$ is definable iff $\mathcal{H}$ is covariantly finite.

Proof. $(\Leftarrow)$ Let $\mathcal{H}$ be covariantly finite and let $\left\{H_{i} ; i \in I\right\}$ be a family of objects of $\mathcal{H}$. Let $\phi: \prod_{i \in I} H_{i} \rightarrow H$ be a left $\mathcal{H}$-approximation. Then the projections $p_{i}: \prod_{i \in I} H_{i} \rightarrow H_{i}$ factor through $\phi$, i.e. there are morphisms $\psi_{i}: H \rightarrow H_{i}$ such that $\phi \circ \psi_{i}=p_{i}, \forall i \in I$. Let $\psi: H \rightarrow \prod_{i \in I} H_{i}$ be the unique morphism such that $\psi \circ p_{i}=\psi_{i}$. It follows directly that $\phi \circ \psi=1 \prod_{i \in I} H_{i}$, so $\prod_{i \in I} H_{i}$ is a direct summand of $H \in \mathcal{H}$. Since $\mathcal{H}$ is closed under direct limits, $\mathcal{H}$ is closed under direct summands. Hence $\prod_{i \in I} H_{i} \in \mathcal{H}$ and $\mathcal{H}$ is closed under products. It follows that $\mathcal{H}$ is definable.

$(\Rightarrow)$ Let $\mathcal{H}$ be definable and fix $M \in \operatorname{Mod}-\mathfrak{C}$. If $\alpha: M \rightarrow H$ is a morphism with $H \in$ $\mathcal{H}$, by the above Lemma, there exists a pure subobject $T_{H} \subseteq H$ such that $\operatorname{Im}(\alpha) \subseteq T_{H}$ and $\left|T_{H}\right| \leqslant \max \left\{\aleph_{0}, \aleph(\mathfrak{C}),|M|\right\}$. Since $\mathcal{H}$ is definable, $T_{H} \in \mathcal{H}$. Consider the set $\mathcal{H}_{M}=$ 
$\left\{G \in \mathcal{H}:|G| \leqslant \max \left\{\aleph_{0}, \aleph(\mathfrak{C}),|M|\right\}\right\}$ and for $G \in \mathcal{H}_{M}$, let $I_{M}:=\operatorname{Mod}-\mathfrak{C}(M, G)$. We set $H^{M}:=\prod_{G \in \mathcal{H}_{M}} G^{I_{M}}$. Since $\mathcal{H}$ is definable, the object $H^{M}$ is in $\mathcal{H}$ and there exists a canonical morphism $\omega: M \rightarrow H^{M}$. By construction $\omega$ is a left $\mathcal{H}$-approximation.

Before we prove our first main result of this section we need to recall some well-known concepts. Recall from [24] that a Grothendieck category $\mathcal{G}$ is called locally Noetherian, resp. locally Artinian, resp. locally finite, if $\mathcal{G}$ has a set of generators consisting of Noetherian, resp. Artinian, resp. finite length, objects. Let $\mathcal{G}$ be an additive category with direct limits. An object $A \in \mathcal{G}$ is called finitely presented if the functor $\mathcal{G}(A,-): \mathcal{G} \rightarrow \mathfrak{A} b$ commutes with direct limits. We denote by f.p. $(\mathcal{G})$ the full subcategory of finitely presented objects. Recall from $[\mathbf{1 6}]$ that an additive category $\mathcal{G}$ is called locally finitely presented if $\mathcal{G}$ has direct limits, f.p. $(\mathcal{G})$ is skeletally small and any object of $\mathcal{G}$ is a direct limit of finitely presented objects. By [16], an additive category $\mathcal{G}$ is locally finitely presented iff $\mathcal{G} \approx \operatorname{Flat}(\operatorname{Mod}-($ f.p.(G)). A Grothendiek category $\mathcal{G}$ is called locally coherent, if $\mathcal{G}$ is locally finitely presented and the full subcategory of finitely presented objects form an abelian category. Finally recall from $[\mathbf{4 2}]$ that a functor category is called perfect, if any flat functor is projective.

Parts (2) and (4) of the following result generalizes results of [18].

Theorem 7.6. (1) FPInj(Mod-C $)$ is covariantly finite.

(2) The following are equivalent:

$(\alpha) \mathfrak{C}$ is left coherent (has cokernels).

( $\beta) \mathfrak{C}$-Mod is locally coherent.

$(\gamma)$ Flat $(\operatorname{Mod}-\mathfrak{C})$ is covariantly finite (reflective) in Mod-C.

$(\delta) \operatorname{FPInj}(\mathfrak{C}-\mathrm{Mod})$ is contravariantly finite (coreflective) in $\mathfrak{C}-\mathrm{Mod}$.

(3) The following are equivalent:

$(\alpha)$ Mod-C $\mathfrak{C}$ is perfect and $\mathfrak{C}$ is left coherent ( $\mathfrak{C}$ has cokernels).

$(\beta) \operatorname{Proj}(\operatorname{Mod}-\mathfrak{C})$ is covariantly finite (reflective) in Mod-C .

If the parenthetical case of part $(\beta)$ is true, then gl.dimMod-c $\leqslant 2$.

(4) The following are equivalent:

( $\alpha$ ) Mod-C is locally Noetherian (and gl.dimMod- $\mathfrak{C} \leqslant 2$ ).

( $\beta) \operatorname{Inj}(\operatorname{Mod}-\mathfrak{C})$ is contravariantly finite (coreflective) in $\operatorname{Mod}-\mathfrak{C}$.

Proof. (1) Since FPInj(Mod-C $)$ is closed under products and pure subobjects, the proof of Proposition 7.5, shows that FPInj(Mod-C $)$ is covariantly finite.

$(2)(\alpha) \Leftrightarrow(\gamma)$ Since obviously Flat $(\operatorname{Mod}-\mathfrak{C})$ is closed under direct limits and pure subobjects, it follows that Flat (Mod-C $)$ is definable iff Flat(Mod-C $)$ is closed under products and by $[\mathbf{3 7}]$, this happens iff $\mathfrak{C}$ is left coherent. Hence the assertion case follows from Proposition 7.5. To prove the parenthetical case of part $(\alpha) \Leftrightarrow(\gamma)$, we proceed as follows (for a different proof see [16]). If Flat $(\operatorname{Mod}-\mathfrak{C})$ is a reflective subcategory, then clearly Flat(Mod-C $)$ has cokernels. Let $f: A \rightarrow B$ be a morphism in $\mathfrak{C}$ and consider the finitely presented functor $M_{f}=\operatorname{Coker} \mathfrak{C}(-, f)$. Let $\tau: M_{f} \rightarrow F$ be the reflection of $C_{f}$ in Flat(Mod- $\left.\mathfrak{C}\right)$. Then the cokernel of $\mathfrak{C}(-, f)$ in Flat $(\operatorname{Mod}-\mathfrak{C})$ is the composition $c_{f} \circ \tau: \mathfrak{C}(-, B) \rightarrow F$, where $c_{f}:=\operatorname{coker} \mathfrak{C}(-, f)$. Let $\left\{F_{i}: i \in I\right\}$ be a filtered system of flat functors. Since the filtered colimit $\underset{\longrightarrow}{\lim } F_{i}$ is a flat functor, the reflection $\tau$ induces isomorphisms $\left(F, \underset{\longrightarrow}{\lim F_{i}}\right) \stackrel{\cong}{\longrightarrow}\left(M_{f}, \underset{\lim F_{i}}{\longrightarrow}\right.$. Since $\overrightarrow{M_{f}}$ is a finitely presented, the functor $\left(M_{f},-\right)$ commutes with filtered colimits. It follows directly that the canonical morphism $\underset{\lim }{\longrightarrow}\left(F, F_{i}\right) \rightarrow\left(F, \underset{\lim }{\longrightarrow} F_{i}\right)$ is an isomorphism. Hence $F$ is a finitely presented object in the locally finitely presented category Flat(Mod- $\mathfrak{C})$. Hence by $[\mathbf{1 6}]$ it is representable, so $F=\mathfrak{C}(-, C)$. By Yoneda there exists a morphism $g: B \rightarrow C$ such that $\mathfrak{C}(-, g)=c_{f} \circ \tau$. It follows directly the $g$ is the cokernel of $f$ in $\mathfrak{C}$. Hence $\mathfrak{C}$ has cokernels.

Conversely assume that $\mathfrak{C}$ has cokernels. We first show that Flat(Mod-cic) has cokernels. Let $\alpha: F \rightarrow G$ be a morphism in Flat (Mod-C $)$. It is well-known that we can write $F=\underset{\lim }{\longrightarrow}\left(X_{\lambda}\right)$, 
$G=\lim \mathbf{P}\left(Y_{\lambda}\right)$ and $\alpha=\lim \mathbf{P}\left(\alpha_{\lambda}\right)$. Consider the exact sequences $X_{\lambda} \stackrel{\alpha_{\lambda}}{\longrightarrow} Y_{\lambda} \rightarrow Z_{\lambda} \rightarrow 0$ in $\mathfrak{C}$. Since direct limits are exact, the sequence $\lim \mathbf{P}\left(X_{\lambda}\right) \rightarrow \lim \mathbf{P}\left(Y_{\lambda}\right) \rightarrow \lim \mathbf{P}\left(Z_{\lambda}\right) \rightarrow 0$ is exact in Flat(Mod-C $)$. Hence Flat(Mod-C $)$ has cokernels. Now let $M$ be in Mod-C and let $P_{1} \stackrel{\alpha}{\rightarrow} P_{0} \stackrel{\epsilon}{\rightarrow} M \rightarrow 0$ be a projective presentation of $M$. Let $\beta: P_{0} \rightarrow F_{M}$ be the cokernel of $\alpha$ in Flat(Mod-cic). There exists a unique morphism $\tau: M \rightarrow F_{M}$ such that $\beta=\epsilon \circ \tau$. We claim that $\tau$ is the flat reflection of $M$. Indeed let $f: M \rightarrow G$ be a morphism with $G$ flat. Then $\alpha \circ \epsilon \circ f=0$, hence there exists a unique morphism $g: F_{M} \rightarrow G$ in Flat(Mod-cic), such that $\epsilon \circ f=\beta \circ g=\epsilon \circ \tau \circ g$. Since $\epsilon$ is epic, we have $f=\tau \circ g$. Clearly $g$ is the unique morphism with this property. This shows that Flat $(\operatorname{Mod}-\mathfrak{C})$ is reflective in Mod-C.

$(\alpha) \Leftrightarrow(\delta)$ We prove only the parenthetical case. First suppose that $\mathfrak{C}$ has cokernels. Then according to Proposition 7.1, $M$ is FP-injective iff $M$ preserves cokernels. It follows directly that the cokernel of a morphism between FP-injective functors is FP-injective. Since FPInj( $(\mathfrak{C}-$ Mod $)$ is closed under coproducts, it follows easily that FPInj( $\mathfrak{C}-$ Mod) has colimits and the inclusion $I:$ FPInj $(\mathfrak{C}-$ Mod $) \hookrightarrow \mathfrak{C}-$ Mod preserves them. A cardinality argument as in $[\mathbf{1 9}]$ p.119, shows that FPInj( $\mathfrak{C}-$ Mod $)$ has also a generating set. So by the Special Adjoint Functor Theorem, FPInj( $\mathfrak{C}-$ Mod) is coreflective. Conversely let FPInj( $\mathfrak{C}-$ Mod $)$ be coreflective. Then FPInj( $\mathfrak{C}-$ Mod $)$ is closed under direct limits, so by $[46] \mathfrak{C}$ is left coherent. Let $0 \rightarrow F_{2} \stackrel{b}{\rightarrow} F_{1} \stackrel{a}{\rightarrow} F_{0}$ be an exact sequence where $F_{0}, F_{1}$ are flat right modules. Then we have the exact sequence $\mathrm{D}\left(F_{0}\right) \stackrel{D(a)}{\longrightarrow} D\left(F_{1}\right) \stackrel{\mathrm{D}(b)}{\longrightarrow} \mathrm{D}\left(F_{2}\right) \rightarrow 0$ in $\mathfrak{C}-$ Mod, where $\mathrm{D}(M)(-)=\mathfrak{A} b(M(-), \mathbb{Q} / \mathbb{Z})$. Obviously $\mathrm{D}\left(F_{0}\right), \mathrm{D}\left(F_{1}\right)$ are FP-injective. Since FPInj( has cokernels, $\mathrm{D}\left(F_{2}\right)$ is FP-injective. Using Proposition 7.1 we see that $F_{2}$ is flat. This implies that w.gl.dim $\mathfrak{C}-$ Mod $\leqslant 2$ or equivalently that for the abelian category $\mathcal{B}(\mathfrak{C})$ we have gl. $\operatorname{dim} \mathcal{B}(\mathfrak{C}) \leqslant 2$. Then by Proposition $4.5, \mathfrak{C}$ has cokernels. Finally the equivalence $(\alpha) \Leftrightarrow(\beta)$ follows from the fact that $\mathfrak{C}-\bmod \approx \mathcal{B}(\mathfrak{C})^{o p}$ which is abelian iff $\mathfrak{C}$ is left coherent.

(3) If Proj(Mod-C $)$ is covariantly finite, then as in the proof of Proposition 7.5, it follows that Proj(Mod-C $)$ is closed under products. By Chase's Theorem (which by standard arguments is true in our setting), $\mathfrak{C}$ is left coherent and Mod- $\mathfrak{C}$ is perfect. Conversely if Mod- $\mathfrak{C}$ is perfect and $\mathfrak{C}$ has weak cokernels, then the assertion follows from (2) since any flat module is projective.

(4) If Mod-C is locally Noetherian, then $\mathfrak{C}$ is right coherent and the injective modules coincide with the FP-injective ones. So by $(2), \operatorname{Inj}(\operatorname{Mod}-\mathfrak{C})$ is contravariantly finite. Conversely if this holds, then it is easy to see that $\operatorname{Inj}(\operatorname{Mod}-\mathfrak{C})$ is closed under coproducts. Hence by [24], Mod- $\mathfrak{C}$ is locally Noetherian. The parenthetical case is easy and is left to the reader.

\subsection{Auslander Categories, the Abelianness of Flat Functors and Consequences}

We recall that Mod-C $\mathfrak{C}$ has weak dominant dimension greater or equal than $n$, notation w.dom.dimMod $-\mathfrak{C} \geqslant n$, if in any injective resolution of any flat module, the first $n$ terms are flat. The next result generalizes results of Stauffer [45] and answers a question of Simson [43], see also [25] for a similar result obtained indepedently.

Theorem 7.7. The following statements are equivalent:

(1) Flat(Mod-C $)$ is an abelian category.

(2) FPInj( $(\mathfrak{C}-$ Mod) is an abelian category.

(3) The following conditions are true:

(a) $\mathfrak{C}$ is left coherent.

(b) w.gl.dimMod $-\mathfrak{C} \leqslant 2$.

(c) w.dom.dimMod- $\mathfrak{C} \geqslant 2$.

Moreover if one of the above equivalent statements is true then Flat(Mod-cic) is a Grothendieck category (hence has injective envelopes and an injective cogenerator) and the injective objects of Flat(Mod-C $)$ are the flat-injective right modules. 
Proof. (1) $\Leftrightarrow(2)$ By Theorem 7.6, Flat(Mod-C $)$ is reflective in Mod-C iff FPInj( $\mathfrak{C}-$ Mod) is coreflective in $\mathfrak{C}-$ Mod. Using this remark the rest is easy and is left to the reader.

$(1) \Rightarrow(3)$ Suppose that Flat $(\operatorname{Mod}-\mathfrak{C})$ is abelian. Since Flat(Mod-cic $)$ has coproducts and a set of generators, and the direct limits are exact since they are computed in Mod- $-\mathfrak{C}$, Flat $(\operatorname{Mod}-\mathfrak{C})$ is in fact a Grothendieck category. Consider the functor $h_{\mathfrak{C}}: \mathfrak{C} \rightarrow$ Flat $(\operatorname{Mod}-\mathfrak{C})$, given by $h_{\mathfrak{C}}(X)=\mathfrak{C}(-, X)$ and let $I:$ Flat $(\operatorname{Mod}-\mathfrak{C}) \rightarrow$ Mod- $-\mathfrak{C}$ be the functor defined by $I(F)=\operatorname{Mod}-\mathfrak{C}\left(h_{\mathfrak{C}}(-), F\right)$. Obviously $I$ is (isomorphic to) the inclusion Flat(Mod- $\left.-\mathfrak{C}\right)$ $\hookrightarrow \operatorname{Mod}-\mathfrak{C}$. By [49], the functor $I$ has a left adjoint $S: \operatorname{Mod}-\mathfrak{C} \rightarrow$ Flat(Mod-C $)$. Hence Flat $(\operatorname{Mod}-\mathfrak{C})$ is a reflective subcategory of Mod-C $\mathfrak{C}$ and by Theorem 7.6 , the category $\mathfrak{C}$ has cokernels. Then by Proposition 4.5 the category $\mathcal{B}(\mathfrak{C})$ is abelian and gl.dimB $\mathcal{B}(\mathfrak{C}) \leqslant 2$. Since $\mathcal{B}(\mathfrak{C}) \approx(\mathfrak{C}-\text { mod })^{o p}$, we have that w.gl.dimMod- $\mathfrak{C} \leqslant 2$. Now let $f: A \rightarrow B$ be a $\mathfrak{C}$-morphism, and consider the exact sequence $0 \rightarrow F \stackrel{\beta}{\rightarrow} \mathfrak{C}(-, A) \stackrel{\mathfrak{C}(-, f)}{\longrightarrow} \mathfrak{C}(-, B)$ in Flat(Mod $-\mathfrak{C})$. Since $F$ is flat, $F$ can be written as a direct limit $F:=\lim \mathfrak{C}\left(-, X_{i}\right)$. Consider the natural epic $\alpha: \oplus \mathfrak{C}\left(-, X_{i}\right) \rightarrow \lim \mathfrak{C}\left(-, X_{i}\right) \rightarrow 0$. Setting $\gamma=\alpha \circ \beta$, we have an exact sequence $\oplus \mathfrak{C}\left(-, X_{i}\right) \stackrel{\gamma}{\rightarrow} \mathfrak{C}(-, A) \stackrel{\mathfrak{C}(-, f)}{\longrightarrow} \mathfrak{C}(-, B)$ in Mod-CC. By Ulmer's Flatness Criterion [49], the left adjoint $S$ of the inclusion $I: \operatorname{Flat}(\operatorname{Mod}-\mathfrak{C}) \hookrightarrow \operatorname{Mod}-\mathfrak{C}$ is exact. Then $\mathcal{U}:=\operatorname{Ker}(S)$ is a localizing subcategory of $\operatorname{Mod}-\mathfrak{C}$, and $(\operatorname{Mod}-\mathfrak{C}) / \mathcal{U} \approx \operatorname{Flat}(\operatorname{Mod}-\mathfrak{C})$. Then the flat modules are exactly the $\mathcal{U}$-closed objects, with respect to the localizing subcategory $\mathcal{U}$. By [39] for any flat module $F$, w.dom.dim $F \geqslant 2$. Hence w.dom.dimMod- $\mathfrak{C} \geqslant 2$.

$(3) \Rightarrow(1)$ Suppose that the conditions $(a),(b),(c)$ are true. Since $\mathfrak{C}$ has weak cokernels, as in the proof of Theorem 7.6, we have that any product of flat functors in Mod-C is flat. Let $\mathcal{W}:=\operatorname{Sub}($ Flat $(\operatorname{Mod}-\mathfrak{C}))$ be the full subcategory of Mod-C generated by the submodules of flat modules. Let $0 \rightarrow G \stackrel{\alpha}{\rightarrow} F$ be an inclusion, where $F \in$ Flat(Mod- $-\mathfrak{C})$, and let $\beta$ : $G \rightarrow E(G), \gamma: F \rightarrow E(F)$ be injective envelopes in Mod-C․ Then there exists a morphism $\delta: E(G) \rightarrow E(F)$ with $\alpha \circ \gamma=\beta \circ \delta$. Since $\beta$ is an essential monic, $\delta$ is monic. Hence $E(G)$ is a direct summand of $E(F)$. By condition $(c), E(F)$ is flat; hence $E(G)$ is flat. We conclude that $\mathcal{W}$ is closed under injective envelopes. Obviously $\mathcal{W}$ is closed under subobjects and since any product of flat functors is flat, $\mathcal{W}$ is closed under products. Using that $\mathcal{W}$ is also closed under injective envelopes, it is easy to see that $\mathcal{W}$ is also closed under extensions. Hence $\mathcal{W}$ is the torsion free subcategory of a torsion theory $(\mathcal{T}, \mathcal{W})$ in $\operatorname{Mod}-\mathfrak{C}$, in which the torsion part $\mathcal{T}$ is a localizing subcategory of Mod-C,$($ see $[\mathbf{3 9}]$ ), and in which obviously any flat-injective is a $\mathcal{T}$-closed object. Since any flat module has weak dominant dimension greater or equal to two, we have by [39], that any flat module is a $\mathcal{T}$-closed object. Now let $F$ be a $\mathcal{T}$-closed object. Then obviously $F \in \mathcal{W}$, hence there exists a short exact sequence (1) $0 \rightarrow F \stackrel{a}{\rightarrow} Q_{0} \stackrel{b}{\rightarrow} H \rightarrow 0$ with $Q_{0}$ a flat module. Consider the canonical short exact sequence $0 \rightarrow H_{1} \stackrel{c}{\rightarrow} H \stackrel{d}{\rightarrow} H_{2} \rightarrow 0$ with $H_{1} \in \mathcal{T}$ and $H_{2} \in \mathcal{W}$. Then the pull-back of (1) along the morphism $c$ factors through $b$ since $F$ is $\mathcal{T}$-closed and $H_{1} \in \mathcal{T}$. Let $e: H_{1} \rightarrow Q_{0}$ be a morphism with $e \circ b=c$; since $c$ is monic also $e$ is monic and then since $H_{1} \in \mathcal{T}$ and $Q_{0} \in \mathcal{W}$, we have $H_{1}=0$. Hence $H \in \mathcal{W}$, and thus there exists a short exact sequence (2) $0 \rightarrow H \stackrel{f}{\rightarrow} Q_{1} \stackrel{g}{\rightarrow} L \rightarrow 0$ with $Q_{1}$ a flat module. Since w.gl.dimMod- $-\mathfrak{C} \leqslant 2$, from the exact sequence $0 \rightarrow F \stackrel{a}{\rightarrow} Q_{0} \stackrel{b \circ f}{\rightarrow} Q_{1}$ we have that $F$ is flat. We conclude that Flat $(\operatorname{Mod}-\mathfrak{C})$ coincides with the full subcategory of $\mathcal{T}$-closed objects. Hence Flat $(\operatorname{Mod}-\mathfrak{C})$ is an abelian category.

If Flat(Mod-C $)$ is abelian then, as observed above, Flat(Mod-cic is in fact a Grothendieck category which is a Giraud subcategory of Mod-C . It follows that Inj(Flat $(\operatorname{Mod}-\mathfrak{C}))$ consists of all flat-injective modules by $[\mathbf{4 7}]$.

As a direct consequence of the above result we have the following, see also [44].

Corollary 7.8. A locally finitely presented additive category $\mathcal{G}$ is abelian (Grothendieck) if and only if f.p. $(\mathcal{G})$ is left coherent, w.gl.dimMod-f.p. $(\mathcal{G}) \leqslant 2$ and w.dom.dimMod-f.p. $(\mathcal{G}) \geqslant 2$. 
Further a locally finitely presented additive category $\mathcal{G}$ is a module category iff f.p. $(\mathcal{G}) \approx \mathcal{A}(\mathfrak{C})$ for an additive category $\mathfrak{C}$. In this case $\mathcal{G} \approx \operatorname{Mod}-\mathfrak{C}$.

There is up to equivalence a bijective correspondence between locally finitely presented abelian categories $\mathcal{G}$ and skeletally small left coherent additive categories $\mathfrak{C}$ with split idempotents such that $\mathrm{w} . \mathrm{gl} \cdot \operatorname{dimMod}-\mathfrak{C} \leqslant 2$ and $\mathrm{w} \cdot \operatorname{dom} \cdot \operatorname{dimMod}-\mathfrak{C} \geqslant 2$. The correspondence is given by:

$$
\mathcal{G} \mapsto \text { f.p. }(\mathcal{G}) \text { and } \mathfrak{C} \mapsto \operatorname{Flat}(\operatorname{Mod}-\mathfrak{C}) .
$$

Corollary 7.9. A skeletally small additive category $\mathfrak{C}$ is abelian iff Flat(Mod-C $)$ is locally coherent iff $\mathfrak{C}$ is coherent, w.gl.dimMod $-\mathfrak{C} \leqslant 2$ and w.dom.dimMod- $\mathfrak{C} \geqslant 2$. If this is the case, then $\mathfrak{C}$ is Noetherian iff Flat(Mod-C $)$ is locally Noetherian. Moreover the maps

$$
\mathfrak{C} \longmapsto \operatorname{Flat}(\operatorname{Mod}-\mathfrak{C}) \quad \text { and } \mathcal{G} \longmapsto \text { f.p. }(\mathcal{G})
$$

are mutually inverse bijections between skeletally small, resp. Noetherian, abelian categories and locally coherent, resp. locally Noetherian, Grothedieck categories.

Proof. This follows from our previous results and the fact that if Flat(Mod-C $)$ is locally coherent, then $\mathfrak{C} \approx$ f.p. $($ Flat $(\operatorname{Mod}-\mathfrak{C}))$ is abelian.

If $\mathfrak{C}$ is a locally finitely presented, then following $[\mathbf{4 1}],[\mathbf{3 1}]$, the conjugate category of $\mathfrak{C}$ is defined by $\widehat{\mathfrak{C}}=\operatorname{Flat}($ f.p. $(\mathfrak{C})-$ Mod $)$. It is easy to see that $\widehat{\mathfrak{C}}=\mathfrak{C}$ and f.p. $(\widehat{\mathfrak{C}})=$ f.p. $(\mathfrak{C})^{o p}$. If $\mathfrak{C}$ is skeletally small, then $\widehat{\operatorname{Mod}}-\mathfrak{C}=\operatorname{Flat}(\mathcal{A}(\mathfrak{C})-\operatorname{Mod})$ and $\mathfrak{C}-\widehat{\operatorname{Mod}}=\operatorname{Flat}(\operatorname{Mod}-\mathcal{B}(\mathfrak{C}))$. We recall from section 4 , that a pair $(\mathfrak{C}, \mathfrak{D})$ of additive categories is called a Morita pair if there exists an equivalence $\mathcal{A}(\mathfrak{C}) \approx \mathcal{B}(\mathfrak{D})$ and a category $\mathfrak{C}$ is called dualizing if $(\mathfrak{C}, \mathfrak{C})$ is a Morita pair.

Lemma 7.10. (i) $(\mathfrak{C}, \mathfrak{D})$ is a Morita pair iff $\mathfrak{D}-\widehat{\operatorname{Mod}} \approx \operatorname{Mod}-\mathfrak{C}$ iff $\widehat{\operatorname{Mod}}-\mathfrak{C} \approx \mathfrak{D}-\operatorname{Mod}$. In particular $\mathfrak{C}$ is dualizing iff $\mathfrak{C}-\widehat{\operatorname{Mod}} \approx \operatorname{Mod}-\mathfrak{C}$ iff $\widehat{\operatorname{Mod}}-\mathfrak{C} \approx \mathfrak{C}-$ Mod.

(ii) Let $(\mathfrak{C}, \mathfrak{D})$ be a Morita pair. Then Mod-C is locally Noetherian iff Mod- $\mathfrak{D}$ is perfect iff $\mathfrak{D}-\operatorname{Mod}$ is locally Artinian. Similarly $\mathfrak{D}-$ Mod is locally Noetherian iff $\mathfrak{C}-\operatorname{Mod}$ is perfect iff Mod-C is locally Artinian. In particular Mod-C is locally finite iff so is $\mathfrak{D}-$ Mod.

(iii) If $\mathfrak{C}$ is dualizing, then Mod-C $\mathfrak{C}$ is perfect (locally Noetherian) iff Mod-C $\mathfrak{C}$ is locally Noetherian (perfect) iff $\mathfrak{C}-\mathrm{Mod}$ is locally Artinian. In particular Mod-c $\mathfrak{C}$ is locally finite iff $\mathfrak{C}-\operatorname{Mod}$ is locally finite.

Proof. If $(\mathfrak{C}, \mathfrak{D})$ is a Morita pair, choose an equivalence $D: \mathcal{A}(\mathfrak{C}) \stackrel{\approx}{\rightarrow} \mathcal{B}(\mathfrak{D})$. Then $\widehat{\operatorname{Mod}}-\mathfrak{C}=$ $\operatorname{Flat}(\mathcal{A}(\mathfrak{C})-\operatorname{Mod}) \approx \operatorname{Flat}(\mathcal{B}(\mathfrak{D})-\operatorname{Mod}) \approx \mathfrak{D}-\operatorname{Mod}$, where the last equivalence follows from Corollary 7.2. Similarly $\mathfrak{D}-\widehat{\operatorname{Mod}} \approx \operatorname{Mod}-\mathfrak{C}$. If $\widehat{\operatorname{Mod}}-\mathfrak{C} \approx \mathfrak{D}-\operatorname{Mod}$, then f.p. $(\widehat{\operatorname{Mod}}-\mathfrak{C}) \approx$ f.p. $(\mathfrak{D}-$ Mod $) \Rightarrow \mathcal{A}(\mathfrak{C})^{o p} \approx \mathcal{B}(\mathfrak{D})^{o p} \Rightarrow \mathcal{A}(\mathfrak{C}) \approx \mathcal{B}(\mathfrak{D})$, so the pair $(\mathfrak{C}, \mathfrak{D})$ is Morita. If this is the case, then $\mathfrak{D}$ is left coherent, so by $[\mathbf{4 1}]$, Mod-C is locally Noetherian iff $\widehat{\operatorname{Mod}}-\mathfrak{C}=\mathfrak{D}-$ Mod is coperfect. Then by $[\mathbf{4 3}], \mathfrak{D}-$ Mod is coperfect iff Mod-D is perfect. Since $\mathcal{A}(\mathfrak{C}) \approx \mathcal{B}(\mathfrak{D})$, we have that Mod-C $\mathfrak{C}$ is locally Noetherian iff $\mathfrak{D}-\operatorname{Mod}$ is locally Artinian. The proof of the other assertions is similar.

The next result generalizes parts of a theorem of Tachikawa [48].

Corollary 7.11. The following statements are equivalent:

(i) $\operatorname{Proj}(\operatorname{Mod}-\mathfrak{C})$ is an abelian category.

(ii) Mod-C is a perfect Auslander category.

If condition (i) or (ii) is true, then $\operatorname{Proj}(\operatorname{Mod}-\mathfrak{C})$ is a Grothendieck category.

Proof. If $\operatorname{Proj}(\operatorname{Mod}-\mathfrak{C})$ is an abelian category, then using the same arguments as in the previous Theorem, we have that $\operatorname{Proj}(\operatorname{Mod}-\mathfrak{C})$ is a reflective subcategory of Mod-C . Hence by Theorem 7.6, Mod-C $\mathfrak{C}$ is perfect. By Theorem 7.7, Mod-C $\mathfrak{C}$ is a perfect Auslander category. The converse also follows from Theorem 7.7. 
For the notion of pure semisimplicity we refer to $[\mathbf{4 3}]$.

Corollary 7.12. The following statements are equivalent:

(i) Mod-C is pure semisimple.

(ii) There exists an equivalence $\mathcal{A}(\operatorname{Mod}-\mathfrak{C}) \approx \operatorname{Mod}-\mathcal{A}(\mathfrak{C})$.

(iii) $\operatorname{Mod}-\mathcal{A}(\mathfrak{C})$ is an Auslander category over a left coherent category.

(iv) $\operatorname{Proj}(\operatorname{Mod}-\mathcal{A}(\mathfrak{C}))$ is an abelian category.

(v) The Auslander category $\mathcal{F}(\mathfrak{C})$ of $\mathfrak{C}$ is Noetherian.

In particular Mod-C $\mathfrak{C}$ and $\mathfrak{C}$-Mod are pure-semisimple iff the Auslander category $\mathcal{F}(\mathfrak{C})$ of $\mathfrak{C}$ is finite length category, i.e. is Artinian and Noetherian.

Proof. (i) $\Rightarrow$ (ii) First note that by $[\mathbf{4 3}]$, Mod-C $\mathfrak{C}$ be pure-semisimple iff Mod- $\mathcal{A}(\mathfrak{C})$ is perfect. If this holds then $\operatorname{Mod}-\mathfrak{C} \approx \operatorname{Flat}(\operatorname{Mod}-\mathcal{A}(\mathfrak{C}))=\operatorname{Proj}(\operatorname{Mod}-\mathcal{A}(\mathfrak{C}))$. Hence $\operatorname{Mod}-\mathcal{A}(\mathfrak{C}) \approx$ $\mathcal{A}(\operatorname{Proj}(\operatorname{Mod}-\mathcal{A}(\mathfrak{C}))) \approx \mathcal{A}(\operatorname{Mod}-\mathfrak{C})$. (ii) $\Rightarrow($ iii $)$ The equivalences $\operatorname{Mod}-\mathcal{A}(\mathfrak{C}) \approx \mathcal{A}(\operatorname{Mod}-\mathfrak{C}) \approx$ $\mathcal{A B}(\operatorname{Inj}(\operatorname{Mod}-\mathfrak{C}))$ show that $\operatorname{Mod}-\mathcal{A}(\mathfrak{C})$ is the Auslander category of $\operatorname{Inj}(\operatorname{Mod}-\mathfrak{C})$, which is obviously left coherent. (iii) $\Rightarrow$ (iv) If $\operatorname{Mod}-\mathcal{A}(\mathfrak{C})$ is Auslander, then it is of the form $\mathcal{A B}(\mathfrak{D})$ where $\mathfrak{D}=\operatorname{Proj} \operatorname{Inj}(\operatorname{Mod}-\mathcal{A}(\mathfrak{C}))$. Since $\operatorname{Proj}(\operatorname{Mod}-\mathcal{A}(\mathfrak{C}))=\operatorname{Proj}(\mathcal{A B}(\mathfrak{D}))=\mathcal{B}(\mathfrak{D})$, and since by hypothesis $\mathfrak{D}$ is left coherent, we have that $\mathcal{B}(\mathfrak{D})$ and consequently $\operatorname{Proj}(\operatorname{Mod}-\mathcal{A}(\mathfrak{C}))$ is abelian. (iv) $\Rightarrow$ (i) By Corollary 7.11, $\operatorname{Mod}-\mathcal{A}(\mathfrak{C})$ is perfect and by [43], Mod- $\mathfrak{C}$ is pure semisimple. $(\mathrm{i}) \Rightarrow(\mathrm{v})$ Since the pair $(\mathcal{B}(\mathfrak{C}), \mathcal{A}(\mathfrak{C}))$ is Morita, Lemma 7.10 implies that Mod- $\mathcal{B}(\mathfrak{C})$ is locally Noetherian iff $\operatorname{Mod}-\mathcal{A}(\mathfrak{C})$ is perfect iff $\operatorname{Mod}-\mathfrak{C}$ is pure semisimple. Then the equivalence (i) $\Leftrightarrow(\mathrm{v})$ follows using that f.p. $(\operatorname{Mod}-\mathcal{B}(\mathfrak{C}))=\bmod -\mathcal{B}(\mathfrak{C}) \approx \mathcal{F}(\mathfrak{C})$.

As an easy consequence we have the following result of Auslander [3].

Corollary 7.13. The following are equivalent

(i) $\mathcal{F}(\mathfrak{C}) \approx$ f.l.Mod $-\Gamma$, where f.l.Mod $-\Gamma$ are the finite length modules over a ring $\Gamma$.

(ii) $(\alpha) \mathfrak{C}$ is a Krull-Schmidt category and $\exists Y \in \mathfrak{C}: \mathfrak{C}=\operatorname{add}(Y)$.

( $\beta)$ The ring $\Lambda:=\operatorname{End}_{\mathfrak{C}}(Y)$ is representation finite.

$(\gamma) \Gamma \cong \operatorname{End}_{\Lambda}\left(\oplus_{i=1}^{n} X_{i}\right)^{o p}$, where $\left\{X_{1}, \ldots, X_{n}\right\}$ is a complete set of non-iso-morphic indecomposable finitely presented right $\Lambda$-modules.

Combining Corollaries 7.11 and 7.12 with the above result we have the following.

Corollary 7.14. There is up to equivalence a bijective correspondence between locally finitely presented pure semisimple abelian (Grothendieck) categories and perfect Auslander module categories. The correspondence is given by:

$$
\mathcal{G} \mapsto \operatorname{Mod}-\text { f.p. }(\mathcal{G}) \text { and } \operatorname{Mod}-\mathfrak{C} \mapsto \operatorname{Proj}(\operatorname{Mod}-\mathfrak{C})
$$

This correspondence induces a bijection between Morita equivalence classes of rings of finite representation type and Artinian Auslander rings.

For any additive category $\mathfrak{C}$ we denote by $\mathcal{I} n d(\mathfrak{C})$ the category of Ind-objects over $\mathfrak{C}$ and by $\operatorname{Pro}(\mathfrak{C})$ the category of Pro-objects over $\mathfrak{C}[\mathbf{2 6}],[\mathbf{4 5}]$. It is well known that $\mathcal{I} n d(\mathfrak{C}) \approx$ Flat $(\operatorname{Mod}-\mathfrak{C})$ and $\mathcal{P r o}(\mathfrak{C}) \approx \operatorname{Flat}(\mathfrak{C}-\operatorname{Mod})^{o p}$, so there exists a duality $\operatorname{Pro}(\mathfrak{C})^{o p} \approx \mathcal{I} n d\left(\mathfrak{C}^{o p}\right)=$ $\widehat{\mathcal{I} n d(\mathfrak{C})}$. Hence Theorem 7.7 gives necessary and sufficient conditions for $\mathcal{I} n d(\mathfrak{C})$ to be an abelian (Grothendieck) category. Moreover we have the following consequence.

Corollary 7.15. The following conditions are equivalent:

(i) $\operatorname{Pro}(\mathfrak{C})$ is an abelian category.

(ii) Pro(C) is a CoGrothendieck category, i.e. its dual is Grothendieck.

(iii) The following conditions are true: 
(a) $\mathfrak{C}$ is right coherent.

(b) w.gl.dimc - Mod $\leqslant 2$.

(c) w.dom.dim $\mathfrak{C}-\mathrm{Mod} \geqslant 2$.

If one of the above statements is true then $\mathcal{P}$ ro $(\mathfrak{C})$ is a bicomplete abelian category with projective covers and exact inverse limits and admits a projective generator. Moreover the category $\operatorname{Proj}(\mathcal{P r o}(\mathfrak{C}))$ coincides with the dual of the full subcategory of flat-injective left $\mathfrak{C}$-modules.

Proof. Obviously (i) $\Leftrightarrow$ (ii). If (i) is true, then $\operatorname{Pro}(\mathfrak{C})^{o p} \approx$ Flat $(\mathfrak{C}-$ Mod) is abelian and (iii) follows from Theorem 7.7. If (iii) holds, then by Theorem 7.7, Flat( $\mathfrak{C}-$ Mod) is Grothendieck, so its dual $\mathcal{P}$ ro $(\mathfrak{C})$ is abelian and the remaining assertions follow.

Corollary 7.16. A skeletally small additive category $\mathfrak{C}$ is abelian iff $\mathcal{P} r o(\mathfrak{C})^{o p}$ is locally coherent iff $\mathfrak{C}$ is coherent and w.gl.dim $\mathfrak{C}-\operatorname{Mod} \leqslant 2$ and w.dom.dim $\mathfrak{C}-\operatorname{Mod} \geqslant 2$. If this is the case, then $\mathfrak{C}$ is Artinian iff $\mathcal{P}$ ro $(\mathfrak{C})^{o p}$ is locally Noetherian. Moreover the maps

$$
\mathfrak{C} \longmapsto \mathcal{P r o}(\mathfrak{C})^{o p} \quad \text { and } \mathcal{G} \longmapsto \text { f.p. }\left(\mathcal{P r o}(\mathfrak{C})^{o p}\right)
$$

are mutually inverse bijections between skeletally small, resp. Artinian, abelian categories and locally coherent, resp. locally Noetherian, categories.

Remark 7.17. If $\mathfrak{C}$ is an abelian category, then by Theorem 7.7 the Ind-category $\operatorname{Ind}(\mathfrak{C})$ is abelian. If moreover $\mathfrak{C}$ is Noetherian then by an old result of Oort, see [38], the inclusion $\mathfrak{C} \hookrightarrow$ $\mathcal{I} n d(\mathfrak{C})$ induces bijections $\mathcal{E} x t_{\mathfrak{C}}^{n}(A, B) \stackrel{\cong}{\longrightarrow} x t_{\mathcal{I}_{n d(\mathfrak{C})}}^{n}(A, B), \forall n \geqslant 0, \forall A, B \in \mathfrak{C}$. This implies by devissage that the inclusion $\mathfrak{C} \hookrightarrow \mathcal{I} n d(\mathfrak{C})$ induces a full exact embedding $\mathcal{D}^{b}(\mathfrak{C}) \hookrightarrow \mathcal{D}^{b}(\operatorname{Ind}(\mathfrak{C}))$ between the corresponding bounded derived categories. Dually if $\mathfrak{C}$ is an Artinian abelian category, then the inclusion $\mathfrak{C} \hookrightarrow \mathcal{P} r o(\mathfrak{C})$ of abelian categories induces a full exact embedding $\mathcal{D}^{b}(\mathfrak{C}) \hookrightarrow \mathcal{D}^{b}(\mathcal{P r o}(\mathfrak{C}))$. These observations can be used to define right derived functors of additive functors $\mathfrak{C} \rightarrow \mathfrak{D}$, if $\mathfrak{C}$ and $\mathfrak{D}$ are Noetherian without injectives, resp. Artinian without projectives. For instance let $\mathfrak{C}$ and $\mathfrak{D}$ be Artinian categories and $F: \mathfrak{C} \rightarrow \mathfrak{D}$ be an additive functor. Since $\operatorname{Pro}(\mathfrak{C})$ has enough projectives, the induced functor $\operatorname{Pro}(F): \mathcal{P r o}(\mathfrak{C}) \rightarrow \operatorname{Pro}(\mathfrak{D})$ admits a left derived functor $\mathbb{L}^{-}(\mathcal{P r o}(F)): \mathcal{D}^{-}(\mathcal{P} r o(\mathfrak{C})) \rightarrow \mathcal{D}^{-}(\mathcal{P r o}(\mathfrak{D}))$, see $[\mathbf{5 1}]$. If the cohomology objects $\mathrm{H}^{n}\left(\mathbb{L}^{-}(\mathcal{P} r o(F)(X))\right)$ lie in the Serre subcategory $\mathfrak{D}$ of Artinian objects of $\mathcal{P} r o(\mathfrak{D}), \forall X \in \mathfrak{C}$, then $F$ admits a left derived functor $\mathbb{L}^{b}(F): \mathcal{D}^{b}(\mathfrak{C}) \rightarrow \mathcal{D}^{b}(\mathfrak{D})$. Dual remarks are applied for functors between Noetherian categories, see $[\mathbf{2 9}]$.

This procedure can be used to define "right derived" functors of additive functors $F: \mathfrak{C} \rightarrow$ $\mathfrak{A} b$, in case the additive category $\mathfrak{C}$ satisfies the conditions of Theorem 7.7, since any such $F$ extends uniquely via the inclusion $\mathfrak{C} \hookrightarrow \mathcal{I} n d(\mathfrak{C})$, to an additive functor $F^{!}: \operatorname{Ind}(\mathfrak{C}) \rightarrow \mathfrak{A} b$ which commutes with filtered colimits. Since $\mathcal{I} n d(\mathfrak{C})$ is a Grothendieck category, the sequence of right derived functors $\mathcal{R}^{*} F^{!}$exists, and we can consider the composite $\mathfrak{C} \hookrightarrow \mathcal{I} n d(\mathfrak{C}) \stackrel{\mathcal{R}^{*} F^{!}}{\longrightarrow} \mathfrak{A} b$ as a sequence of right derived functors of $F$. Dual remarks are applied for left derived functors.

Another direct consequence of Theorems 7.6, 7.7, Corollary 7.15 and its dual, is the following classical result which leads to the full imbedding theorem.

Corollary 7.18. If $\mathfrak{C}$ is an abelian category, then the categories $\operatorname{Lex}\left(\mathfrak{C}^{\circ o p}, \mathfrak{A} b\right)$, Lex $(\mathfrak{C}, \mathfrak{A} b)$ are Giraud and the categories $\operatorname{Rex}\left(\mathfrak{C}^{o p}, \mathfrak{A} b\right), \operatorname{Rex}(\mathfrak{C}, \mathfrak{A} b)$ are co-Giraud subcategories of Mod-C $\mathfrak{C}$, $\mathfrak{C}-$ Mod respectively. The category $\mathfrak{C}$ has full exact imbeddings and is closed under extensions in $\operatorname{Lex}\left(\mathfrak{C}^{o p}, \mathfrak{A} b\right), \operatorname{Lex}(\mathfrak{C}, \mathfrak{A} b)$.

More generally any additive category $\mathfrak{C}$ which satisfies the conditions of Theorem 7.7, resp. of Corollary 7.15, admits a full embedding into a module category, which preserves the existing exact structure.

We close this section showing that in case $\mathfrak{C}$ is coherent, the abelianness of the category of flat modules over $\mathfrak{C}$ is a symmetric condition, see also [44]. 
Theorem 7.19. If $\mathfrak{C}$ is a skeletally small additive category, then the following are equivalent:

(i) $\mathfrak{C}$ is right coherent and Flat $(\operatorname{Mod}-\mathfrak{C})$ is abelian.

(ii) $\mathfrak{C}$ is left coherent and Flat $(\mathfrak{C}-M o d)$ is abelian.

(iii) The categories $\mathcal{I}$ nd $(\mathfrak{C})$ and $\mathcal{P}$ ro( $(\mathfrak{C})$ are abelian.

(iv) $\mathfrak{C}$ is an abelian category.

Proof. (i) $\Leftrightarrow$ (iii) If $\mathfrak{C}$ is right coherent and Flat $(\mathfrak{C}-$ Mod) is abelian, then by Corollary 7.9, $\mathfrak{C}$ is abelian. Conversely if $\mathfrak{C}$ is abelian, then obviously $\mathfrak{C}$ is left and right coherent. Moreover is this case Flat(Mod-C $)$ is identified with the category Lex $\left(\mathfrak{C}^{o p}, \mathfrak{A} b\right)$ of left exact functors $\mathfrak{C}^{o p} \rightarrow \mathfrak{A} b$. Hence Flat (Mod-cic) is is abelian by Corollary 7.9. The proof of (ii) $\Leftrightarrow$ (iv) follows by duality and the proof of (iii) $\Leftrightarrow$ (iv) follows by our previous results.

¿From Corollaries 7.9 and 7.11, we have the following, see also [44] for a related result.

Corollary 7.20. Let $\mathfrak{C}$ be a coherent category. If $\mathfrak{C}-\mathrm{Mod}$ and $\mathrm{Mod}-\mathfrak{C}$ are perfect, then the following conditions are equivalent:

(i) Mod-C is an Auslander category.

(ii) $\mathfrak{C}-$ Mod is an Auslander category.

We leave to the reader to state and prove the analogous results of Corollary 7.9, Theorem 7.11, for the case of FP-injective or injective modules, and we refer to the work of Simson and Garcia [44] for further related results.

\section{Free Homological, Triangulated and Weak Abelian Categories}

In this section we study the Freyd categories of (left or right) triangulated, and more generally, of weak abelian categories.

\subsection{Free Homological Categories}

Suppose that $(\mathfrak{C}, \Omega, \Delta)$ is a left triangulated category. We recall that an additive functor $H$ : $\mathfrak{C} \rightarrow \mathcal{D}$ to an abelian category $\mathcal{D}$ is called homological, if for any triangle $\Omega(C) \stackrel{h}{\rightarrow} A \stackrel{g}{\rightarrow} B \stackrel{f}{\rightarrow}$

$C \in \Delta$, the sequence $H(A) \stackrel{H(g)}{\longrightarrow} H(B) \stackrel{H(f)}{\longrightarrow} H(C)$ is exact in $\mathcal{D}$. A cohomological functor is a contravariant homological functor. (Co-)homological functors from (right) triangulated categories are defined similarly.

A free homological category over a left (right) triangulated category $(\mathfrak{C}, \Omega, \Delta)$, is a pair $(H, \mathcal{H})$ where $\mathcal{H}$ is an abelian category and $H: \mathfrak{C} \rightarrow \mathcal{H}$ is a homological functor, satisfying the following universal property:

$(\dagger)$ if $F: \mathfrak{C} \rightarrow \mathfrak{D}$ is homological functor to an abelian category $\mathfrak{D}$, then there exists a unique exact functor $F^{\star}: \mathcal{H} \rightarrow \mathfrak{D}$, with the property $F^{\star} H=F$.

Theorem 8.1. If $\mathfrak{C}$ is a left (resp. right) triangulated category then the pair $\left(\mathbf{P}_{\mathfrak{C}}, \mathcal{A}(\mathfrak{C})\right.$ ) (resp. $\left.\left(\mathbf{Q}_{\mathfrak{C}}, \mathcal{B}(\mathfrak{C})\right)\right)$ is the free $(c o)$-homological category over $\mathfrak{C}$. Any left (resp. right) triangulated category is fully embedded via a homological functor to an abelian category with enough projectives (resp. injectives) as the full subcategory of projective (resp. injective) objects.

Proof. Since $\mathfrak{C}$ is left triangulated, any morphism $f: B \rightarrow C$ is embedded in a triangle $\Omega(C) \stackrel{h}{\rightarrow} A \stackrel{g}{\rightarrow} B \stackrel{f}{\rightarrow} C \in \Delta$, which trivially can be extended to a weak-kernel sequence over the morphism $f$. Hence any morphism in $\mathfrak{C}$ has a weak kernel and by Proposition $4.5, \mathcal{A}(\mathfrak{C})$ is abelian. By Corollary 4.7, the functor $\mathbf{P}_{\mathfrak{C}}: \mathfrak{C} \rightarrow \mathcal{A}(\mathfrak{C})$ is homological. If $F: \mathfrak{C} \rightarrow \mathfrak{D}$ is a homological functor to an abelian category $\mathfrak{D}$, then from Corollary 4.7, the functor $F^{!}$: $\mathcal{A}(\mathfrak{C}) \rightarrow \mathfrak{D}$ is the unique exact functor with $F^{!} \mathbf{P}_{\mathfrak{C}}=F$. The rest follows from Proposition 3.6 ( $\mathfrak{C}$ as always has split idempotents). The parenthetical case is dual. 
Corollary 8.2. Let $(\mathfrak{C}, \Omega, \Delta)$ be a left triangulated category. If $\Omega^{n}=0$, then gl.dim $\mathcal{A}(\mathfrak{C}) \leqslant$ $3 \cdot n-1$. More generally if $\mathfrak{C}$ is $\Omega$-regular in the sense that $\forall A \in \mathfrak{C}, \exists n_{A} \geqslant 0: \Omega^{n_{A}}(A)=0$, then $\mathcal{A}(\mathfrak{C})$ is homologically regular in the sense that any object has finite projective dimension. If $\Omega$ is full and faithful, then $\operatorname{gl} \cdot \operatorname{dim} \mathcal{A}(\mathfrak{C})=0$ or $\infty$. In particular if $\mathfrak{C}$ is abelian with enough projectives (injectives), and gl.dim $\mathfrak{C} \leqslant n$, then $\operatorname{gl} \cdot \operatorname{dim} \mathcal{A}(\underline{\mathfrak{C}})=\operatorname{gl} \cdot \operatorname{dim} \mathcal{B}(\overline{\mathfrak{C}}) \leqslant 3 \cdot n-1$.

Proof. Consider the exact endofunctor

$$
\mathcal{A}(\Omega): \mathcal{A}(\mathfrak{C}) \rightarrow \mathcal{A}(\mathfrak{C}), \quad \mathcal{A}(\omega)\{A, f, B\}=\{\Omega(A), \Omega(f), \Omega(B)\} .
$$

¿From the projective resolution of $\{A, f, B\}$ induced by a triangle with base morphism $f$, we see that $\{\Omega(A), \Omega(f), \Omega(B)\}$ is a third syzygy. Inductively $\mathcal{A}(\Omega)^{n}\{A, f, B\}$ is a $3 \cdot n$-syzygy of $\{A, f, B\}$. Hence if $\Omega^{n}=0$, then gl.dim $\mathcal{A}(\mathfrak{C}) \leqslant 3 \cdot n-1$. Obviously p.d $\{A, f, B\}<\infty$ $\Leftrightarrow \exists n \geqslant 0:\left\{\Omega^{n}(A), \Omega^{n}(f), \Omega^{n}(B)\right\}$ is projective. But $\left\{\Omega^{n}(A), \Omega^{n}(f), \Omega^{n}(B)\right\}$ is projective $\Leftrightarrow$ $\exists g: \Omega^{n}(B) \rightarrow \Omega^{n}(A)$ with $\Omega^{n}(f) \circ g \circ \Omega^{n}(f)=\Omega^{n}(f)$. If $\Omega$ is fully faithful this implies that $f \circ h \circ f=f$, where $\Omega^{n}(h)=g$ and this means that $\{A, f, B\}$ is projective. Since any object of finite projective dimension is projective, we see that $\operatorname{gl} \operatorname{dim} \mathcal{A}(\mathfrak{C})=0$ or $\infty$.

Corollary 8.3. If $(\mathfrak{C}, \Omega, \Delta)$ is triangulated, then the pairs $\left(\mathbf{P}_{\mathfrak{C}}, \mathcal{A}(\mathfrak{C})\right),\left(\mathbf{Q}_{\mathfrak{C}}, \mathcal{B}(\mathfrak{C})\right)$ are free homological categories over $\mathfrak{C}$, and there exists a unique equivalence $D: \mathcal{A}(\mathfrak{C}) \stackrel{\approx}{\sim} \mathcal{B}(\mathfrak{C})$ with $D \mathbf{P}_{\mathfrak{C}}=\mathbf{Q}_{\mathfrak{C}}$. The Freyd category $\mathcal{A}(\mathfrak{C})$ is Frobenius, and any triangulated category is imbedded via a homological functor to a Frobenius abelian category as the full subcategory of projectiveinjective objects.

Proof. Since $\mathfrak{C}$ can be considered as a left and right triangulated category, the first part follows from Theorem 8.1. From the universal property of the free homological category, it

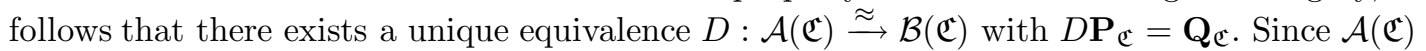
has projectives and $\mathcal{B}(\mathfrak{C})$ has injectives, the equivalence $D$ and the relation $D \mathbf{P}_{\mathfrak{C}}=\mathbf{Q}_{\mathfrak{C}}$ show that $\mathcal{A}(\mathfrak{C})$ has projectives and injectives, and the projectives and the injectives coincide.

¿From Corollary 8.3 we see that a triangulated category is dualizing with Frobenius abelian Freyd categories. Hence gl.dim $\mathcal{A}(\mathfrak{C})=\operatorname{gl} \cdot \operatorname{dim} \mathcal{B}(\mathfrak{C})=0$ or $\infty$. Trivially gl.dim $\mathcal{A}(\mathfrak{C})=0$ iff the triangulation $\Delta$ of $\mathfrak{C}$ consists only of split triangles, where a triangle is called split if it is isomorphic to a triangle of the form $\Omega(C) \stackrel{0}{\rightarrow} A \rightarrow A \oplus B \rightarrow B$.

Let $\Delta_{0} \subseteq \Delta$ be the full subcategory of split triangles. The next result describes the stable category $\Delta / \Delta_{0}$.

Proposition 8.4. If $(\mathfrak{C}, \Omega, \Delta)$ is triangulated, then $\left(\Delta, \Delta_{0}\right)$ is a homotopy pair, the stable category $\Delta / \Delta_{0}$ is triangulated and there are triangle equivalences:

$$
\underline{\mathcal{A}(\mathfrak{C})} \stackrel{\approx}{\longrightarrow} \Delta / \Delta_{0} \stackrel{\approx}{\mathcal{B}(\mathfrak{C})}
$$

Proof. We leave to the reader the proof that $\left(\Delta, \Delta_{0}\right)$ is a homotopy pair, noting only that the loop functor of $\Delta / \Delta_{0}$ is the first rotation of the triangles in $\Delta$, when we view these in $\Delta / \Delta_{0}$. Now define a functor $\tilde{F}: \Delta \rightarrow \mathcal{A}(\mathfrak{C})$ as follows. $\tilde{F}(E)=\{B, f, C\}$, if $E:=\Omega(C) \stackrel{h}{\rightarrow} A \stackrel{g}{\rightarrow} B \stackrel{f}{\rightarrow}$ $C \in \Delta$, and $F(a, b, c)=\{b, c\}$. From the axioms of a triangulated category, the functor $\tilde{F}$ is full and dense. Hence composing $\tilde{F}$ with the functor $\varpi: \mathcal{A}(\mathfrak{C}) \rightarrow \mathcal{A}(\mathfrak{C})$, we obtain a full and surjective on objects functor $F: \Delta \rightarrow \mathcal{A}(\mathfrak{C})$. Suppose that $(a, b, c): \overline{\left(E_{1}\right)} \rightarrow\left(E_{2}\right)$ is a morphism of triangles, where $\left(E_{i}\right): \Omega\left(C_{i}\right) \stackrel{h_{i}}{\longrightarrow} \overline{A_{i} \stackrel{g_{i}}{\longrightarrow}} B_{i} \stackrel{f_{i}}{\longrightarrow} C_{i}, i=1,2$, and let $F(a, b, c)=0$. Then the morphism $\{b, c\}:\left\{B_{1}, f_{1}, C_{1}\right\} \rightarrow\left\{B_{2}, f_{2}, C_{2}\right\}$ factors through the morphism $\left\{0,1_{C_{2}}\right\}$ : $\mathbf{P}\left(C_{2}\right) \rightarrow\left\{B_{2}, f_{2}, C_{2}\right\}$. Analyzing this situation we see that $\exists n: C_{1} \rightarrow C_{2}, m: C_{1} \rightarrow B_{2}$, such that: $c-n=m \circ f_{2}, f_{1} \circ n=0$. Hence $c=m \circ f_{2}+n$. Then $f_{1} \circ c=f_{1} \circ m \circ f_{2}+f_{1} \circ n \Rightarrow$ $b \circ f_{2}=f_{1} \circ m \circ f_{2} \Rightarrow\left(b-f_{1} \circ m\right)=0 \Rightarrow b-f_{1} \circ m=t \circ g_{2}$, since $g_{2}$ is a weak kernel of $f_{2}$. Hence $b=f_{1} \circ m+t \circ g_{2}$. This implies that the morphism $(a, b, c)$ is homotopic to zero, when we view the triangles $\left(E_{i}\right)$ as complexes. It is not difficult to see that this implies that 
$(a, b, c)$ factors through the right $\Delta_{0}$-approximation of $\left(E_{2}\right)$. Thus $(a, b, c)$ is zero in $\Delta / \Delta_{0}$. Since $F$ vanishes on $\Delta_{0}$, there is an induced full dense functor $\tilde{F}: \Delta / \Delta_{0} \rightarrow \mathcal{A}(\mathfrak{C})$, and the above argument shows that $\tilde{F}$ is an equivalence. Since $\mathcal{A}(\mathfrak{C})$ is Frobenius, the stable category $\underline{\mathcal{A}(\mathfrak{C})}$ is triangulated $[\mathbf{1 0}]$. The easy proof that $\tilde{F}$ preserves triangles is left to the reader.

In [35] it is proved that if we consider the triangles of a triangulated category as (short) 3-periodic complexes, then the cone of a morphism of triangles is not necessarily a triangle. However from Proposition 8.4 we deduce easily the following.

Corollary 8.5. If $(\mathfrak{C}, \Omega, \Delta)$ is triangulated and $\left(E_{1}\right) \rightarrow\left(E_{2}\right)$ is a morphism of triangles, then there exists a split triangle $\left(E_{0}\right)$, such that $\left(E_{0}\right) \oplus$ Cone $\left\{\left(E_{1}\right) \rightarrow\left(E_{2}\right)\right\}$ is a triangle. If $F: \mathfrak{C} \rightarrow \mathfrak{D}$ is a (co-)homological functor to an abelian category, then $F$ induces a long exact sequence in $\mathfrak{D}$, if we apply $F$ to Cone $\left\{\left(E_{1}\right) \rightarrow\left(E_{2}\right)\right\}$.

\subsection{Weak Abelian Categories}

Definition 8.6. An additive category $\mathfrak{C}$ is called weak abelian if $\mathfrak{C}$ is coherent and any morphism in $\mathfrak{C}$ is a weak kernel and a weak cokernel.

A sequence $\cdots \rightarrow A_{i-1} \stackrel{f_{i-1}}{\longrightarrow} A_{i} \stackrel{f_{i}}{\longrightarrow} A_{i+1} \rightarrow \cdots$ in a weak abelian category $\mathfrak{C}$ is called weak exact iff $f_{i-1}$ is a weak kernel of $f_{i}$ and $f_{i}$ is a weak cokernel $f_{i-1}$. A homological functor $H: \mathfrak{C} \rightarrow \mathfrak{D}$ from a weak abelian category $\mathfrak{C}$ to an abelian category $\mathfrak{D}$, is an additive functor sending weak exact sequences in $\mathfrak{C}$ to exact sequences in $\mathfrak{D}$. If $\mathfrak{C}, \mathfrak{D}$ are weak abelian categories, then a functor $F: \mathfrak{C} \rightarrow \mathfrak{D}$ is called weak exact if $F$ preserves weak exact sequences.

If $\mathfrak{C}$ is a weak abelian category, then a free homological category over $\mathfrak{C}$ is a pair $(H, \Phi(\mathfrak{C}))$ consisting of a homological functor $H: \mathfrak{C} \rightarrow \Phi(\mathfrak{C})$ to an abelian category $\Phi(\mathfrak{C})$, such that for any homological functor $F$ to an abelian category $\mathfrak{D}$, there exists a unique exact functor $G: \Phi(\mathfrak{C}) \rightarrow \mathfrak{D}$ such that $G H=F$

The following theorem contains a simple proof of a result of Freyd [20], (see also [28]). We note that Freyd's description of the free homological category of a weak abelian category is slightly different.

Theorem 8.7. (i) If $\mathfrak{C}$ is a weak abelian category, then the pairs $\left(\mathbf{P}_{\mathfrak{C}}, \mathcal{A}(\mathfrak{C})\right),\left(\mathbf{Q}_{\mathfrak{C}}, \mathcal{B}(\mathfrak{C})\right)$ are free homological categories over $\mathfrak{C}$ and there exists a unique equivalence $D: \mathcal{A}(\mathfrak{C}) \stackrel{\approx}{\longrightarrow} \mathcal{B}(\mathfrak{C})$ with $D \mathbf{P}_{\mathfrak{C}}=\mathbf{Q}_{\mathfrak{C}}$. In particular $\mathfrak{C}$ is dualizing and the free homological category over $\mathfrak{C}$ is Frobenius abelian. Moreover for an arbitrary category $\Phi$ the following are equivalent:

$(\alpha) \Phi$ is a free homological category.

( $\beta) \Phi$ is a Frobenius abelian category.

If one of the above equivalent statements is true, then $\Phi \approx \mathcal{A}(\mathfrak{C}) \approx \mathcal{B}(\mathfrak{C})$, where $\mathfrak{C}$ is the full subcategory of projective-injective objects of $\Phi$.

(ii) For an additive category $\mathfrak{C}$ the following are equivalent:

$(\alpha) \mathcal{A}(\mathfrak{C})$ is a Frobenius (functor) module category.

$(\beta) \mathfrak{C}$ is a weak abelian category with coproducts and a (set of) compact split generator $(s)$.

$(\gamma)$ There exists a QF-ring $\Lambda$ and an equivalence $\mathfrak{C} \approx \operatorname{Proj}(\operatorname{Mod}-\Lambda)$.

Proof. Part (i) follows from the previous results and part (ii) follows from Proposition 4.15. We note only that the category of projectives = injectives in a Frobenius abelian category trivially form a weak abelian category.

By the above Theorem, any triangulated category is weak abelian.

Corollary 8.8. Any weak abelian category is balanced. 
Proof. Let $f: A \rightarrow B$ be monic and epic. Then $\mathbf{P}(f)$ is monic and $\mathbf{Q}(f)$ is epic. By Theorem 8.7, there exists an equivalence $D: \mathcal{A}(\mathfrak{C}) \stackrel{\approx}{\longrightarrow} \mathcal{B}(\mathfrak{C})$ with $D \mathbf{P}_{\mathfrak{C}}=\mathbf{Q}_{\mathfrak{C}}$. This implies that $\mathbf{P}(f)$ is a bimorphism in $\mathcal{A}(\mathfrak{C})$, hence $\mathbf{P}(f)$ is an isomorphism, since $\mathcal{A}(\mathfrak{C})$ is abelian. Since $\mathbf{P}$ is fully faithful, $f$ is an isomorphism in $\mathfrak{C}$.

¿From the full exact embedding Theorem, we have the following.

Corollary 8.9. If $\mathfrak{C}$ is a skeletally small weak abelian category, then there exists a ring $\Lambda$ and a full homological embedding $H: \mathfrak{C} \hookrightarrow \operatorname{Mod}-\Lambda$.

Lemma 8.10. Let $\mathfrak{C}$ be a skeletally small additive category.

(1) If $\mathfrak{C}$ is left coherent, the following are equivalent:

(i) Any morphism in $\mathfrak{C}$ is a weak kernel.

(ii) $\operatorname{Flat}(\mathfrak{C}-\operatorname{Mod}) \subseteq \operatorname{FPInj}(\mathfrak{C}-\operatorname{Mod})$.

(iii) FPInj $(\operatorname{Mod}-\mathfrak{C}) \subseteq$ Flat $(\operatorname{Mod}-\mathfrak{C})$

(iv) $\operatorname{Inj}(\operatorname{Mod}-\mathfrak{C}) \subseteq$ Flat $(\operatorname{Mod}-\mathfrak{C})$.

(2) If $\mathfrak{C}$ is right coherent, the following are equivalent:

(i) Any morphism in $\mathfrak{C}$ is a weak cokernel.

(ii) Flat $(\operatorname{Mod}-\mathfrak{C}) \subseteq$ FPInj(Mod-C $)$.

(iii) $\operatorname{FPInj}(\mathfrak{C}-\operatorname{Mod}) \subseteq \operatorname{Flat}(\mathfrak{C}-\operatorname{Mod})$.

(iv) $\operatorname{Inj}(\mathfrak{C}-\operatorname{Mod}) \subseteq \operatorname{Flat}(\mathfrak{C}-\operatorname{Mod})$.

Proof. (1) We fix a morphism $f: A \rightarrow B$ in $\mathfrak{C}$ with weak cokernel $g: B \rightarrow C$. Below we use the following easy observation:

(*) if $f$ is a weak kernel, then $f$ is a weak kernel of $g$ and $\mathfrak{C}(-, A) \rightarrow \mathfrak{C}(-, B) \rightarrow \mathfrak{C}(-, C) \rightarrow$ $H \rightarrow 0$ is the beginning of a projective resolution of $H:=\operatorname{Coker}(-, g)$.

(i) $\Leftrightarrow$ (ii) Let $F \in \mathfrak{C}$-Mod and $G \in \mathfrak{C}-\bmod$, where $G=\operatorname{Coker}(f,-)$ for a morphism $f: A \rightarrow B$ in $\mathfrak{C}$ with weak cokernel $g: B \rightarrow C$. Let $H:=\operatorname{Coker}(-, g) \in \bmod -\mathfrak{C}$. Using observation $(*)$ above, $\mathcal{E} x t^{1}[G, F]=\operatorname{Ker}(F g) / \operatorname{Im}(F f)$. Hence $\mathcal{T}_{\text {or }}[$ [H, F] $=\operatorname{Ker}(F g) / \operatorname{Im}(F f)=$ $\mathcal{E} x t^{1}[G, F]$. Thus if $F$ is flat then since $\mathfrak{C}$ is left coherent, $F$ is FP-injective. Conversely since $\forall X \in \mathfrak{C}, \mathfrak{C}(X,-)$ is FP-injective, if $A \stackrel{f}{\rightarrow} B \stackrel{g}{\rightarrow} C$ is a weak cokernel diagram, then $\mathcal{E} x t^{1}[G, \mathfrak{C}(X,-)]=0$ where $G=\operatorname{Coker}(f,-)$, which implies that $\mathfrak{C}(-, A) \rightarrow \mathfrak{C}(-, B) \rightarrow$ $\mathfrak{C}(-, C)$ is exact, i.e. $f$ is a weak kernel of $g$.

(i) $\Leftrightarrow$ (iii) Let $F \in \operatorname{Mod}-\mathfrak{C}$ and $G \in \mathfrak{C}-\bmod$, where $G=\operatorname{Coker}(f,-)$ for a morphism $f: A \rightarrow B$ in $\mathfrak{C}$ with weak cokernel $g: B \rightarrow C$. If $H:=\operatorname{Coker}(-, g) \in \bmod -\mathfrak{C}$, then using observation $(*)$ above, we have $\mathcal{T}$ or $_{1}[F, G]=\operatorname{Ker}(F f) / \operatorname{Im}(F g)=\mathcal{E} x t^{1}[H, F]$. Now if $F$ is FP-injective, then since $\mathfrak{C}$ is left coherent, we have $\mathcal{T}$ or $_{i}[F, G]=0, \forall i \geqslant 1, \forall G \in \mathfrak{C}-\bmod$, so $F$ is flat. Conversely since $\forall X \in \mathfrak{C}$, the character module $\operatorname{DC}(X,-)=\mathfrak{A} b(\mathfrak{C}(X,-), \mathbb{Q} / \mathbb{Z})$ is FP-injective, $\mathrm{D} \mathfrak{C}(X,-)$ is flat. Hence if $A \stackrel{f}{\rightarrow} B \stackrel{g}{\rightarrow} C$ is a weak cokernel diagram, then $0=\mathcal{T o r}_{i}[\operatorname{DC}(X,-), \operatorname{Coker}(f,-)]=$ the homology of the complex $\operatorname{DC}(X, C) \rightarrow \operatorname{DC}(X, B) \rightarrow$ $\mathrm{D} \mathfrak{C}(X, A)$. Since D reflects exactness, the complex $\mathfrak{C}(X, A) \rightarrow \mathfrak{C}(X, B) \rightarrow \mathfrak{C}(X, C)$ is exact $\forall X \in \mathfrak{C}$, so $f$ is a weak kernel of $g$.

(iii) $\Leftrightarrow$ (iv) The direction (iii) $\Rightarrow$ (iv) is trivial. Let $f: A \rightarrow B$ be a morphism in $\mathfrak{C}$ with weak cokernel $g: B \rightarrow C$. For any $X \in \mathfrak{C}$, the character module $\operatorname{DC}(X,-)$ is injective, hence by (iv) is flat. Then as in the proof of (iii) $\Leftrightarrow$ (ii), $f$ is a weak kernel of $g$. So (i) and consequently (iii) is true. (2) Similar to the proof of (1).

The next Proposition contains a useful characterization of weak abelian categories in the skeletally small case (see also Corollary 4.9 for another one in the general case).

Proposition 8.11. For a skeletally small additive category $\mathfrak{C}$, the following are equivalent:

(i) $\mathfrak{C}$ is weak abelian. 
(ii) $\operatorname{Flat}(\operatorname{Mod}-\mathfrak{C})=$ FPInj $(\operatorname{Mod}-\mathfrak{C})$.

(iii) $\operatorname{Flat}(\mathfrak{C}-$ Mod $)=\operatorname{FPInj}(\mathfrak{C}-$ Mod $)$.

(iv) $\operatorname{Inj}(\operatorname{Mod}-\mathfrak{C}) \subseteq \operatorname{Flat}(\operatorname{Mod}-\mathfrak{C})$ and $\operatorname{Inj}(\mathfrak{C}-\operatorname{Mod}) \subseteq \operatorname{Flat}(\mathfrak{C}-\operatorname{Mod})$.

(v) $\mathfrak{C}$ is coherent and $\operatorname{Inj}(\operatorname{Mod}-\mathfrak{C}) \subseteq$ Flat $(\operatorname{Mod}-\mathfrak{C})$.

(vi) $\mathfrak{C}$ is coherent and $\operatorname{Inj}(\mathfrak{C}-$ Mod $) \subseteq$ Flat $(\mathfrak{C}-$ Mod $)$.

Proof. By Lemma 8.10, (i) implies (ii), (iii), (iv), (v), (vi). If Flat(Mod-cic) = FPInj(Mod-C $)$, then obviously Flat $(\operatorname{Mod}-\mathfrak{C})$ is closed under products and FPInj(Mod-C $)$ is closed under direct limits. Hence by $[\mathbf{3 7}], \mathfrak{C}$ is left coherent and by $[\mathbf{4 6}], \mathfrak{C}$ is right coherent. Then (ii) $\Rightarrow$ (i) follows from the above Lemma. The rest of the proof is similar using the above Lemma.

Remark 8.12. If $\mathfrak{C}$ is triangulated, let $\mathcal{C} o h\left(\mathfrak{C}^{o p}, \mathfrak{A} b\right)$ be the category of cohomological functors $\mathfrak{C}^{o p} \rightarrow \mathfrak{A} b$, and $\mathcal{H o m}(\mathfrak{C}, \mathfrak{A} b)$ the category of homological functors $\mathfrak{C} \rightarrow \mathfrak{A} b$. Since any triangulated category is weak abelian, by Proposition 7.1, we have the following identifications:

$$
\begin{aligned}
& \mathcal{C} \text { oh }\left(\mathfrak{C}^{\mathfrak{o p}}, \mathfrak{A} b\right)=\operatorname{FPInj}(\operatorname{Mod}-\mathfrak{C})=\operatorname{Flat}(\operatorname{Mod}-\mathfrak{C}) \\
& \mathcal{H} \text { om }(\mathfrak{C}, \mathfrak{A} b)=\operatorname{Flat}(\mathfrak{C}-\operatorname{Mod})=\operatorname{FPInj}(\mathfrak{C}-\operatorname{Mod}) .
\end{aligned}
$$

The next result which follows directly from Lemma 8.10, characterizes the weak abelian categories such that their Freyd categories have finite global dimension.

Corollary 8.13. For the category $\mathfrak{C}$ the following are equivalent:

(i) $\mathfrak{C}$ is von Neumann regular.

(ii) The functor $\mathbf{P}: \mathfrak{C} \rightarrow \mathcal{A}(\mathfrak{C})$ is an equivalence.

(iii) The functor $\mathbf{Q}: \mathfrak{C} \rightarrow \mathcal{B}(\mathfrak{C})$ is an equivalence.

(iv) $\operatorname{FPInj}(\operatorname{Mod}-\mathfrak{C})=\operatorname{Mod}-\mathfrak{C}$.

(v) Flat $(\operatorname{Mod}-\mathfrak{C})=\operatorname{Mod}-\mathfrak{C}$.

(vi) Flat $(\operatorname{Mod}-\mathfrak{C})$ is abelian and any injective module is flat.

(vii) $\mathfrak{C}$ is weak abelian and $\operatorname{gl} \operatorname{dim} \mathcal{A}(\mathfrak{C})<\infty$.

Any one of the above is also equivalent to its dual, replacing $\mathfrak{C}$ by $\mathfrak{C}^{o p}$. If (i) holds, then $\mathfrak{C}$ is semisimple abelian iff Mod-C is locally Noetherian or locally Artinian or perfect.

We have seen that any weak abelian category has Frobenius Freyd categories. The next result characterizes the categories with Frobenius module categories.

Theorem 8.14. For a skeletally small additive category $\mathfrak{C}$, the following are equivalent:

(i) Mod-C is Frobenius.

(ii) $\mathfrak{C}$ is weak abelian and $\mathcal{A}(\mathfrak{C})$, or equivalently $\mathcal{B}(\mathfrak{C})$, is Noetherian.

(iii) $\mathfrak{C}$ is weak abelian and $\mathrm{Mod}-\mathfrak{C}$ is locally Noetherian or equivalently perfect, or equivalently $\mathfrak{C}-$ Mod is locally Artinian.

(iv) $\mathfrak{C}$ is weak abelian and $\operatorname{Proj}(\operatorname{Mod}-\mathfrak{C})$, resp. $\operatorname{Inj}(\operatorname{Mod}-\mathfrak{C})$, is closed under coproducts, resp. products.

Proof. (i) $\Rightarrow$ (ii) Since a coproduct of injectives is injective, Mod-C $\mathfrak{C}$ is locally Noetherian or equivalently mod-C $\approx \mathcal{A}(\mathfrak{C})$ is a Noetherian abelian category. In particular $\mathfrak{C}$ is right coherent. Let $F \in$ Flat (Mod-C $)$; then since $F$ is a direct limit of representables and Mod-C is Frobenius, it follows that $F$ is injective. This implies trivially that projective, injective and flat functors coincide. Hence a product of flat functors is flat and this implies that $\mathfrak{C}$ is left coherent. So $\mathfrak{C}$ is coherent and then by Proposition 8.11, $\mathfrak{C}$ is weak abelian.

(ii) $\Rightarrow$ (i) Since $\mathfrak{C}$ is weak abelian, $\mathfrak{C}$ is coherent and by Proposition 8.11 , Flat $(\operatorname{Mod}-\mathfrak{C})=$ FPInj(Mod- $-\mathfrak{C})$. Since $\mathcal{A}(\mathfrak{C})$ is Noetherian, Mod- $-\mathfrak{C}$ is locally Noetherian and the injectives 
coincide with the FP-injective modules. It follows that Flat $(\operatorname{Mod}-\mathfrak{C})=\operatorname{Inj}(\operatorname{Mod}-\mathfrak{C})$. By Lemma 7.10, Mod-C is perfect, hence $\operatorname{Proj}(\operatorname{Mod}-\mathfrak{C})=\operatorname{Flat}(\operatorname{Mod}-\mathfrak{C})=\operatorname{Inj}(\operatorname{Mod}-\mathfrak{C})$ and Mod $-\mathfrak{C}$ is Frobenius. Since weak abelian categories are dualizing, the parenthetical case and the remaining equivalences follow directly from Lemma 7.10 and Theorem 7.6.

Remark 8.15. By a well-known result of Faith-Walker, for a ring $\Lambda$, the category Mod- $\Lambda$ is a Frobenius category iff $\Lambda$-Mod is a Frobenius category iff $\Lambda$ is a Quasi-Frobenius ring. In this case Mod $-\Lambda, \Lambda-$ Mod are locally finite. However by $[\mathbf{1 2}]$ there exists a skeletally small additive category $\mathfrak{C}$ such that Mod- $\mathfrak{C}$ is Frobenius, but $\mathfrak{C}-$ Mod is not. In particular there exists a Frobenius module category Mod-C $\mathfrak{C}$, which is not locally finite, thus giving a negative answer to a problem of Roos [41]. We refer to [12] for details.

Recall that a ring $\Lambda$ is called right self FP-injective if $\Lambda_{\Lambda}$ is FP-injective. $\Lambda$ is called self FP-injective iff $\Lambda$ is right and left self FP-injective. Also $\Lambda$ is called a right IF-ring if every right injective module is flat, and $\Lambda$ is called an IF-ring if $\Lambda$ is a left and right IF-ring [15]. The following is a direct consequence of Proposition 8.11 and generalizes results of $[\mathbf{4 6}],[\mathbf{1 5}]$.

Corollary 8.16. For a ring $\Lambda$ the folowing are equivalent:

(i) $\mathcal{P}_{\Lambda}$ is weak abelian, or equivalently $\bmod -\Lambda$ is Frobenius.

(ii) $\mathcal{P}_{\Lambda^{\text {op }}}$ is weak abelian, or equivalently $\Lambda$-mod is Frobenius.

(iii) $\Lambda$ is an IF-ring.

(iv) $\Lambda$ is a coherent self FP-injective ring.

(v) $\Lambda$ is coherent and any finitely presented left and right module is reflexive.

In particular: $\Lambda$ is a QF-ring iff $\Lambda$ is right Noetherian left coherent ring and any finitely presented left and right module is reflexive iff $\Lambda$ is a right perfect (right Artinian) left coherent left self FP-injective ring iff $\Lambda$ is a left perfect (left Artinian) right coherent right self FPinjective ring iff $\Lambda$ is a left Noetherian right IF-ring iff $\Lambda$ is a right Noetherian left IF-ring.

We recall from section 7 that for an additive category $\mathfrak{C}$ its category of Ind-objects $\mathcal{I} n d(\mathfrak{C})$ is defined as the full subcategory Flat $(\operatorname{Mod}-\mathfrak{C})$ of $\operatorname{Mod}-\mathfrak{C}$, its category of Pro-objects $\operatorname{Pro}(\mathfrak{C})$ is defined as Flat $(\mathfrak{C}-\operatorname{Mod})^{o p}$ and we have: $\mathcal{I} n d(\mathcal{A}(\mathfrak{C}))=\operatorname{Mod}-\mathfrak{C}$ and $\mathcal{P r o}(\mathcal{B}(\mathfrak{C}))=(\mathfrak{C}-\operatorname{Mod})^{o p}$.

If $F: \mathfrak{C} \rightarrow \mathfrak{D}$ is an additive functor between abelian categories, then in general the right derived functor $\mathbb{R}^{*}(F): \mathcal{D}^{*}(\mathfrak{C}) \rightarrow \mathcal{D}^{*}(\mathfrak{D})$ à la Deligne, see [17], does not exists. The reason is that the image of $\mathbb{R}^{*}(F)$ lies always in $\operatorname{Ind}\left(\mathcal{D}^{*}(\mathfrak{D})\right.$ ), but not necessarily in its full subcategory $\mathcal{D}^{*}(\mathfrak{D})$. Hence it is useful to know when the Ind-category of a triangulated, or more generally weak abelian, category is triangulated. The next result presents a class of categories with the property that their Ind-categories are triangulated. For further results in this direction we refer to $[\mathbf{1 2}]$.

Theorem 8.17. Let $\mathfrak{C}$ be a weak abelian category and suppose that the Auslander category $\mathcal{F}(\mathfrak{C})$ is Noetherian. Then $\mathcal{I} n d(\mathfrak{C})$ is a covariantly finite subcategory of $\mathcal{I} n d(\mathcal{A}(\mathfrak{C}))$, the stable category $\operatorname{Ind}(\mathcal{A}(\mathfrak{C})) / \mathcal{I} n d(\mathfrak{C})$ is triangulated and there exists an equivalence:

$$
\operatorname{Ind}(\mathcal{A}(\mathfrak{C})) / \operatorname{Ind}(\mathfrak{C}) \stackrel{\approx}{\longrightarrow} \operatorname{Ind}(\underline{\mathcal{A}(\mathfrak{C})}) .
$$

Proof. Since $\mathfrak{C}$ is weak abelian, $\mathfrak{C}$ is left coherent, so by Theorem $7.6, \mathcal{I} n d(\mathfrak{C})$ is covariantly finite in $\operatorname{Ind}(\mathcal{A}(\mathfrak{C}))=\operatorname{Mod}-\mathfrak{C}$. By Theorem 2.2 , the stable category $\operatorname{Ind}(\mathcal{A}(\mathfrak{C})) / \mathcal{I} n d(\mathfrak{C})$ is right triangulated. Since $\mathcal{F}(\mathfrak{C})=\bmod -\mathcal{B}(\mathfrak{C})$ is Noetherian by $[\mathbf{2 4}], \operatorname{Mod}-\mathcal{B}(\mathfrak{C}) \approx \operatorname{Mod}-\mathcal{A}(\mathfrak{C})$ is locally Noetherian. Hence its localized quotient Mod-C is also locally Noetherian. By Theorem 8.14, the category Mod-C $\mathfrak{C}$ is Frobenius and $\mathcal{I} n d(\mathfrak{C})=\operatorname{Inj}(\operatorname{Mod}-\mathfrak{C})$. Hence the category $\operatorname{Ind}(\mathcal{A}(\mathfrak{C})) / \mathcal{I} n d(\mathfrak{C})$ is triangulated (see $[\mathbf{1 0}])$.

Since $\mathfrak{C}$ is weak abelian, by Theorem $8.7, \mathcal{A}(\mathfrak{C})$ is Frobenius, so by $[\mathbf{1 0}], \mathcal{A}(\mathfrak{C})$ is triangulated. In particular $\mathcal{A}(\mathfrak{C})$ is weak abelian. Since $\operatorname{Mod}-\mathcal{A}(\mathfrak{C})$ is locally Noetherian as a localizing subcategory of the locally Noetherian category $\operatorname{Mod}-\mathcal{A}(\mathfrak{C})$ we deduce that $\operatorname{Mod}-\mathcal{A}(\mathfrak{C})$ is 
Frobenius. Hence $\mathcal{I} n d(\mathcal{A}(\mathfrak{C}))=\operatorname{Inj}(\operatorname{Mod}-\mathcal{A}(\mathfrak{C}))$. It follows from [24] (see also $[\mathbf{3 2}])$ that the quotient functor $\operatorname{Mod}-\overline{\mathcal{A}(\mathfrak{C})} \rightarrow \operatorname{Mod}-\mathfrak{C}$ induces an equivalence between the stable category $\operatorname{Inj}(\operatorname{Mod}-\mathcal{A}(\mathfrak{C})) / \operatorname{Inj}(\operatorname{Mod}-\mathfrak{C})$ and the category $\operatorname{Inj}(\operatorname{Mod}-\mathcal{A}(\mathfrak{C}))$. But since $\mathcal{A}(\mathfrak{C}) \approx \mathcal{B}(\mathfrak{C})$ and $\operatorname{Mod}-\mathcal{A}(\mathfrak{C})$ is locally Noetherian we can identify $\operatorname{Inj}(\operatorname{Mod}-\mathcal{A}(\mathfrak{C}))=\operatorname{FPInj}(\operatorname{Mod}-\mathcal{A}(\mathfrak{C}))=$ FPInj $(\operatorname{Mod}-\mathcal{B}(\mathfrak{C}))$. Since $\operatorname{Mod}-\mathcal{A}(\mathfrak{C}), \operatorname{Mod}-\mathfrak{C}$ are Frobenius we have also the identifications $\operatorname{Inj}(\operatorname{Mod}-\mathfrak{C})=\mathcal{I} n d(\mathfrak{C}), \operatorname{Inj}(\operatorname{Mod}-\mathcal{A}(\mathfrak{C}))=\mathcal{I} n d(\mathcal{A}(\mathfrak{C}))$. Using the above remarks and Corol-

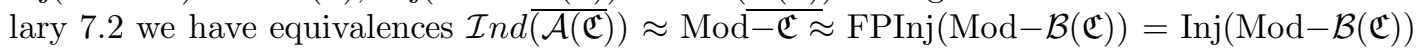
$\approx \operatorname{Inj}(\operatorname{Mod}-\mathcal{A}(\mathfrak{C}))$. Hence the equivalence $\operatorname{Inj}(\operatorname{Mod}-\mathcal{A}(\mathfrak{C})) / \operatorname{Inj}(\operatorname{Mod}-\mathfrak{C}) \approx \operatorname{Inj}(\operatorname{Mod}-\mathcal{A}(\mathfrak{C}))$ induces the desired equivalence $\operatorname{Ind}(\mathcal{A}(\mathfrak{C})) / \mathcal{I} n d(\mathfrak{C}) \approx \mathcal{I} n d(\underline{\mathcal{A}(\mathfrak{C})})$.

Corollary 8.18. $(\alpha)$ Let $\mathfrak{C}$ be a weak abelian category. If the Auslander category $\mathcal{F}(\mathfrak{C})$ is Artinian, then the Pro-category $\operatorname{Pro}(\mathcal{A}(\mathfrak{C}))$ is triangulated.

$(\beta)$ Let $\mathfrak{C}$ be a weak abelian category such that its Auslander category $\mathcal{F}(\mathfrak{C})$ is a finite length category. Then the categories $\operatorname{Pro}(\mathcal{A}(\mathfrak{C})), \mathcal{I} n d(\mathcal{A}(\mathfrak{C}))$ are triangulated.

Proof. $(\alpha) \mathfrak{C}^{o p}$ is weak abelian because $\mathfrak{C}$ is weak abelian. It is easy to see that the equivalence $\mathcal{A}\left(\mathfrak{C}^{o p}\right) \approx \mathcal{B}(\mathfrak{C})^{o p} \approx \mathcal{A}(\mathfrak{C})^{o p}$ of Proposition 3.6, induces an equivalence $\mathcal{A}(\mathfrak{C})^{o p} \approx \mathcal{A}\left(\mathfrak{C}^{o p}\right)$. Hence $\mathcal{P r o}(\mathcal{A}(\mathfrak{C}))=\operatorname{Ind}\left(\mathcal{A}(\mathfrak{C})^{o p}\right)^{o p}=\operatorname{Ind}\left(\mathcal{A}\left(\mathfrak{C}^{o p}\right)\right)^{o p}$. Since the dual of a triangulated category is triangulated, by Theorem 8.17 it remains to show that $\mathcal{F}\left(\mathfrak{C}^{o p}\right)$ is Noetherian. But since $\mathcal{F}(\mathfrak{C})$ is Artinian, $\mathcal{F}\left(\mathfrak{C}^{o p}\right)=\mathcal{F}(\mathfrak{C})^{o p}$ is Noetherian. $(\beta)$ follows from $(\alpha)$ and Theorem 8.14.

Corollary 8.19. If $\mathfrak{C}$ is triangulated and $\mathrm{Mod}-\mathfrak{C}$ is pure-semisimple, then the stable module categories $\underline{\bmod }-\mathfrak{C}, \underline{\operatorname{Mod}}-\mathfrak{C}, \underline{\operatorname{Mod}}-\underline{\mathcal{A}(\mathfrak{C})}$ and the Ind-category $\mathcal{I} n d(\underline{\mathcal{A}(\mathfrak{C})})$ are triangulated.

The interpretation of the above results in the case of the module category of a ring leads to the following result first proved in $[\mathbf{3 3}]$.

Corollary 8.20. If $\Lambda$ is a QF-ring of finite representation type, then the stable module categories $\underline{\bmod }-\Lambda, \underline{\operatorname{Mod}}-\Lambda, \underline{\operatorname{Mod}}-(\underline{\bmod }-\Lambda)$ modulo projectives, and the Ind-, Pro-categories Ind $(\underline{\bmod }-\Lambda)$, Pro $(\underline{\bmod }-\Lambda)$, are triangulated.

Acknowledgement. I would like to thank Prof. Daniel Simson for pointing out to me his joint paper with J.L. Garcia [44] and for some useful discussions.

\section{References}

[1] M. Adelman, Abelian Categories Over Additive Ones, J. Pure Appl. Algebra 3 (1973), $103-117$.

[2] M. Auslander, Representation Dimension of Artin Algebras, Queen Mary College Mathematical Notes, London (1971).

[3] M. Auslander, Representation Theory Of Artin Algebras I, II, Comm. in Algebra 1 (1974), 177-268, 269-310.

[4] M. Auslander, Functors and morphisms determined by objects, Lecture Notes in Pure and Applied Math. Vol. 37, Marcel Dekker, (1978), 1-244.

[5] M. Auslander and M. Bridger, Stable Module Theory, Memoirs of A.M.S. 94 (1969).

[6] M. Auslander and I. Reiten, Stable Equivalence Of Dualizing R-Varieties, Advances in Math. 12 (1974), 306-366.

[7] M. Auslander and I. Reiten, Grothendieck Groups of algebras and orders, J. Pure Appl. Algebra 39 (1986), 1-51.

[8] M. Auslander, I. Reiten and S.O. Smalø, Representation Theory of Artin Algebras, Cambridge studies in advanced mathematics 36 (1994), Cambridge University Press. 
[9] M. Auslander And S.O. Smal $\varnothing$, Preprojective modules over artin algebras, J. Algebra 66 (1980), 61-122.

[10] A. Beligiannnis And N. Marmaridis, Left Triangulated Categories Arising from Contravariantly Finite Subcategories, Comm. Algebra 22 (1994), 5021-5036.

[11] A. Beligiannis, The Homological Theory of Contravariantly Finite Subcategories: Auslander-Buchweitz Contexts, Gorenstein Categories and (Co-)Stabilization, Comm. Algebra 28 (10) (2000), 4547-4596.

[12] A. Beligiannis, Relative Homological Algebra and Purity in Triangulated Categories, J. Algebra 227 No.1 (2000), 268-361.

[13] A. Beligiannis, Homotopy Theory of Modules and Gorenstein Rings, Preprint, University of Bielefeld, (1998). To appear in Math. Scandinavica.

[14] A. Beligiannis, The Auslander Dimension of an Abelian Category, in preparation.

[15] R.R. Colby, Rings Which Have Flat Injective Modules, J. Algebra 35 (1975), 239-252.

[16] W. Crawley- Boevey, Locally Finitely Presented Additive Categories, Comm. Algebra 22 (1994), 1644-1674.

[17] P. Deligne, Cohomologie à Supports Propres, in: SGA 4, Springer Lecture Notes in Mathematics, vol. 305 (1972), 252-480.

[18] E.E. Enochs, Injective and Flat Covers, Envelopes and Resolvents, Israel J. Math. 39 (1981), 189-209.

[19] P.J. Freyd, Abelian Categories, Harper and Row, New York, (1964).

[20] P.J. FREYD, Representations in Abelian Categories, in Proceeding of the Conference on Categorical Algebra (1966), 95-120.

[21] P.J. Freyd, Stable Homotopy, Proc. Conf. on Categorical Algebra, La Jolla 1965, Springer-Verlag, New York (1966), 121-172.

[22] M. FARBER, Von Neumann Categories and Extended L ${ }^{2}$ Cohomology, K-Theory 15 (1998), 347-405.

[23] P. GABriel, Sur les Catégories Abéliennes Localement Noethériennes et leurs Applications aux Algèbres étudiées par Dieudonné, Séminaire J.P. Serre, Collégee de France (1960), 118pp.

[24] P. Gabriel, Des Catégories Abéliennes, Bull. Soc. Math. France 90 (1962), 323-448.

[25] J.L. Garcia And J. Martinez, When is the Category of Flat Modules Abelian?, Fund. Math. 147 (1995), 83-91.

[26] A. Grothendieck and J.L. Verdier, Prefaiscaux in: Théorie des Topos et Cohomologie Etale des Schémas, Springer L.N.M. 269 (1972), 1-217.

[27] L. Gruson And C.U. Jensen, Dimensions cohomologiques relieés aux foncteurs $\lim ^{(i)}$, Springer L.N.M. 867 (1981), 234-294.

[28] A. Heller, Stable Homotopy Categories, Bulletin A.M.S. 74, (1968), 28-63.

[29] A. Huber, Calculation of Derived Functors via Ind-Categories, J. Pure Appl. Algebra 90 (1993), 39-48.

[30] B. Keller And D. Vossieck, Sous les Categories Derivees, C.R. Acad. Sc. Paris 305 (1987), 225-228.

[31] H. Krause, Functors on Locally Finitely Presented Categories, Coll. Math. 75 (1998), 105-131.

[32] H. Krause, Exactly Definable Categories, J. Algebra 201 (1998), 456-492.

[33] H. Krause, Stable Module Categories Preserves Representation Type, Comment. Math. Helv. 72 (1997), 266-284. 
[34] B. Mitchell, Theory of Categories, New York/London: Academic Press, (1965).

[35] A. NeEman, Some new axioms for triangulated categories, J. Algebra 139 (1991), 221255 .

[36] A. NeEman, The Grothendieck Duality Theorem via Bousfield's Techniques and Brown Representability, J. Amer. Math. Soc. 9 (1996), 205-237.

[37] U. Oberst And H. RohrL, Flat and Coherent Functors, J. Algebra 14 (1970), 91-105.

[38] F. Oort, Yoneda Extensions in Abelian Categories, Math. Annalen 153 (1964), 227235.

[39] N. Popescu, Abelian Categories with Applications to Rings and Modules, L.M.S. Monographs 3 (1973), Academic Press.

[40] D. Quillen, Higher K-Theory I, Springer L.N.M. 341 (1973), 85-147.

[41] J.E. Roos, Locally Noetherian Categories and Strictly Linearly Compact Rings, Springer L.N.M. 92 (1969), 197-277.

[42] D. Simson, Functor Categories in which any flat object is projective, Bull. Acad. Polon. Math. 22 (1974), 3755-380.

[43] D. Simson, On pure global dimension of locally finitely presented Grothendieck categories, Fund. Math. 96 (1977), 5-30.

[44] D. Simson And J.L. Garcia, On Rings whose Flat Modules form a Grothendieck Category, Colloq. Math. 73 (1997), 115-141.

[45] H.B. Stauffer, The Completion of an Abelian Category, Trans. A.M.S. 170 (1972), 403-414.

[46] B. Stenstrom, Coherent Rings and FP-Injective Modules, J. London Math. Soc. 2 (1970), 323-329.

[47] B. Stenstrom, Ring and Modules of Quotients, Springer (1975).

[48] H. Tachikawa, QF-3 Rings and Categories of Projective Modules, J. Algebra 28 (1974), 408-413.

[49] F. Ulmer, A Flatness Criterion for Grothendieck Categories, Inventiones Math. 19 (1973), 331-336.

[50] J.L. Verdier, Catégories Dérivées: état 0, in SGA $4 \frac{1}{2}$, Springer Lecture Notes in Mathematics 569 (1977), 262-311.

[51] C.A. Weibel, An Introduction to Homological Algebra, Cambridge Studies in Advanced Mathematics 38 (1994).

Apostolos Beligiannis abeligia@aegean.gr and abeligia@mail.otenet.gr

Department of Mathematics,

University of the Aegean,

83200 Karlovassi,

Samos,

Greece. 